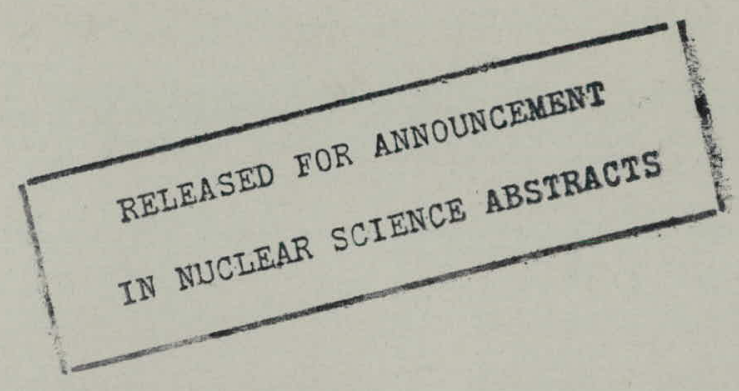

RECEIVED

$A \cup G<\forall 1966$

PATENT GROUP (BROOKGARVEN

MASSACHUSETTS INSTITUTE OF TECHNOLOGY

Laboratory for Nuclear Science

\title{
PHYSICS PROGRESS REPORT
}

December 31, 1965

M. I. T. - 2098 - No. 251

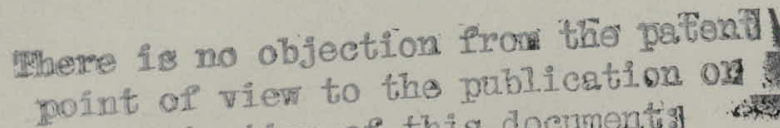

disserination of this dooumentis

Patent Group (Brookbareal)

exec

$8 \longdiv { 2 9 } . 7 9 6 6$ 


\section{DISCLAIMER}

This report was prepared as an account of work sponsored by an agency of the United States Government. Neither the United States Government nor any agency Thereof, nor any of their employees, makes any warranty, express or implied, or assumes any legal liability or responsibility for the accuracy, completeness, or usefulness of any information, apparatus, product, or process disclosed, or represents that its use would not infringe privately owned rights. Reference herein to any specific commercial product, process, or service by trade name, trademark, manufacturer, or otherwise does not necessarily constitute or imply its endorsement, recommendation, or favoring by the United States Government or any agency thereof. The views and opinions of authors expressed herein do not necessarily state or reflect those of the United States Government or any agency thereof. 


\section{DISCLAIMER}

Portions of this document may be illegible in electronic image products. Images are produced from the best available original document. 

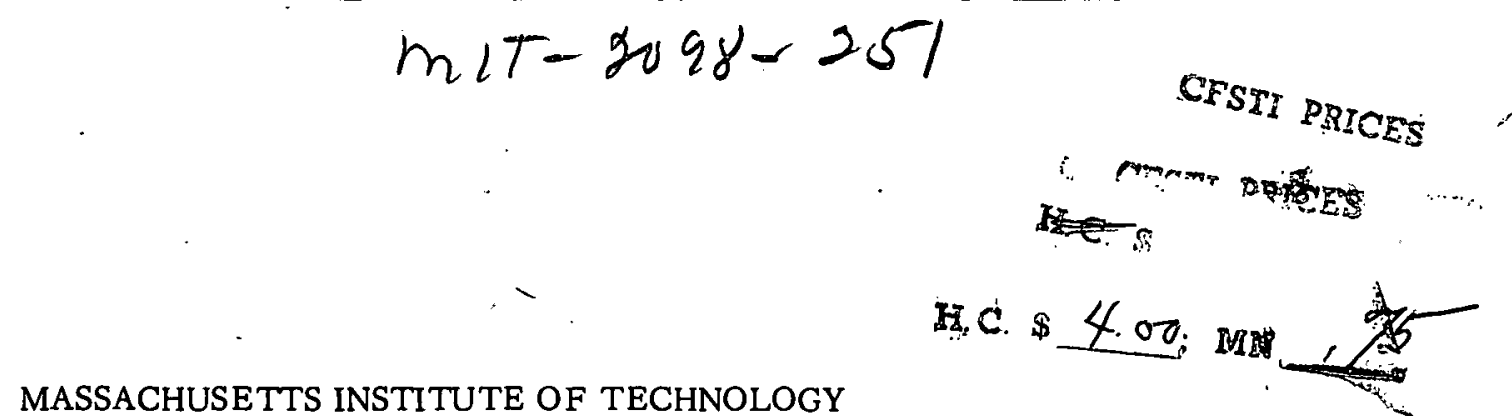

w. C. $\$$ 4.00.

MASSACHUSETTS INSTITUTE OF TECHNOLOGY

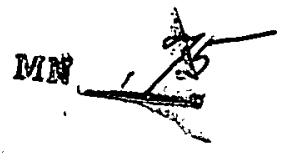

\section{LABORATORY FOR NUCLEAR SCIENCE}

RELEASED FOR ANNOUNCEMENT

IN NUCLEAR SCIENCE ABSTRACTS

\section{PHYSICS P ROGRESS REPORT}

December 31, 1965

M.I. T. $-2098-$ No. 251

\section{LEGAL NOTICE}

This report was prepared as an account of Government sponsored work. Neither the United States, nor the Commission, nor any person acting on behalf of the Commission: A. Makes any warranty or representation, expressed or implied, with respect to the accuracy, completeness, or usefulness of the Information contained in this report, or that the use of any information, apparatus, method, or process disclosed in this report may not infringe privately owned rigbts; or

B. Assumes any llabilities with respect to the use of or for dariages resulting from the use of any information, apparatus, method, or process disclosed in this report.

As used in the above, "person acting on behalf of the Commlssion" includes any employee or contractor of the Commission, or employee of such contractor, to the extent that such employee or contractor of the Commission, or employee of such contractor prepares, disseminates, or provides access to, any information pursuant to his employment or contract with the Commission, or his employment with such contractor.

Reproduction in whole or in part is permitted for any purpose by the U.S. Government. The data and the results that are presented in this report should not otherwise be published without prior consultation with the Laboratory for Nuclear Science.

Researches reported herein have been supported by and large by the Atomic Energy Commission. through AEC contract AT(30-1) -2098 and in part by separate contracts and grants with the Air Force Office of Aerospace Research (contract AF19(628) -2389); the National Aeronautics and Space Administration (contracts NASw -37, NASA -2053, NAS5-3205, grant number NsG-386) and the National Science Foundation (grant number G-19728). The work of the Laboratory's Chemistry groups under Atomic Energy Commission contract AT(30-1)-905 is now reported separately. 
This is the sixty-seventh progress report of the Laboratory for Nuclear Science at the Massachusetts Institute of Technology. Due to the CEA accident the May and November 1965 reports were not issued. Accordingly this report covers the period November 1, 1964 through December 31, 1965.

\section{TAB LE OF CONTENTS}

Cosmic Ray Group. . . . . . . . . . . . . . . 1

Accelerator Physics Collaboration . . . . . . . . 14

Linear Accelerator Group . . . . . . . . . . . 16

High Energy Accelerator Physics Group : . . . . . . . .21

ONR Generator Group. . . . . . . . . . . . . 37

Radioactivity Group. . . . . . . . . . . 50

Cyclotron Group . . . . . . . . . . . . . . 57

Theoretical Group. . . . . . . . . . . . . 74

P ersonnel Listing. . . . . . . . . . . . . . 104

Reprints of published papers listed herein are available in limited numbers upon request from the Laboratory for Nuclear Science Document Room. 


\section{X-Ray Astronomy}

\section{A. Rocket Experiments}

Activity in the new field of $x$-ray astronomy grew substantially during the past year. A major part of the efforts of several members of the group was devoted to participation in the preparation and analysis of several rocket experiments of American Science and Engineering, Inc. Among the new results reported in joint ASE-MIT publications were discoveries of new sources, and measurements of source positions and sizes. For these experiments the MIT group designed and fabricated several new versions of their modulation collimator, which was first developed in this laboratory and has proven to be a powerful and versatile device for the study of cosmic $x$-ray sources.

P reliminary work on the development of instrumentation for an MIT rocket experiment was carried out in anticipation of the receipt of support for a flight during the coming year. The problems of fabricating proportional detectors with ultra thin windows for soft $\mathrm{x}$-rays.were. investigated, and new collimator systems for high resolution size and position measurements . were developed. (H. Bradt, E. Boughan, G. Clark, G. Garmire, W. Mayer, M. Oda, G. Spada, and B. V. Sreekantan)

\section{B. Balloon Experiments}

The apparatus used in the first balloon observation of cosmic $x$-rays in July 1964 was rebuilt and flown several times during the spring and summer of 1965 . Of a total of four flights, three were unsuccessful due to balloon failures and one yielded data, which are being analyzed.

In view of the growing opportunities and interest in balloon $\mathrm{x}$-ray astronomy an expanded program of experiments has been undertaken, which is aimed at the following broad objectives:

(a) General survey of the sky for high energy ( $>15 \mathrm{keV}$ ) $\mathrm{x}$-ray sources.

(b) Study of the spectra, sizes and positions of specific sources.

(c) Search for extra-galactic sources.

Among the instruments being developed for these experiments are proportional detectors with sensitive areas of $700 \mathrm{~cm}^{2}$ and $5000 \mathrm{~cm}^{2}$, and scintillation counter telescopes with telemetric orientation control. (G. Clark, I. Glass, J. Overbeck, W. Smith, B. V. Sreekantan, J. Stein, R. Sullivan and A. Womack)

\section{High Energy Gamma Ray Astronomy}

A. OSO-C P rogram

The OSO-C spacecraft. carrying the MIT gamma ray telescope was launched in August 1965 and failed to orbit. A new spacecraft has been authorized that will carry the backup model 
of the MIT experiment. Meanwhile preparations for the analysis of the anticipated data were completed, and tests on the instrument performance were carried out with a beam of tagged gamma rays at the California Institute of Technology synchrotron. (W. Kraushaar, G. Clark, G. Garmire, M. Greenberg, H. Hinteregger, R. Homonoff, W. Hooker)

B. OAO-A P rogram

During August of 1964, MIT was invited by Dr. James Kupperian to participate in the OAOA program as a backup experiment for the Smithsonian Astrophysical Observatory's U.V. detection system. That system had encountered severe technical difficulties, which involved substantial delays in the program before they could be corrected. Rather than delay the program, it was decided to alter the spacecraft structure to accommodate several "off the shelf" experiments, which could be readied on short notice.

The backup system for Explorer XI was such an experiment and required only minor modifications to adapt it to the OAO structure and electronics. This effort was carried out jointly by MIT's Laboratory for Nuclear Science and the Center for Space Research and completed by January $1,1965$.

The OAO-A is now scheduled for launch in the spring of 1966.

Data Analysis

The data analysis scheme will be similar in principle to that of the OSO-C experiment. Engineering data transmitted in the routine mode will be.extracted from the binary data tape together with the analogue data and displayed in graphical form so that the performance of the instrument can be continuously monitored. The data for each individual gamma ray event will be extracted and listed on separate IBM cards. Celestial exposure will be evaluated by the random event technique that has been used for Explorer XI and OSO-C. The results will be presented in the form of charts and maps giving the intensity of gamma ray events as functions of parameters such as geomagnetic latitude, right ascension and declination, galactic latitude, etc.

Work on the data analysis program was begun in June on the basis of preliminary informa tion about the OAO data formats obtained from the Goddard group. (W. Kraushaar, G. Clark, G. Garmire, M. Greenberg, R. Homonoff, W. Hooker)

\section{Digitized Spark Chamber for Gamma Ray Astronomy}

During the first six months of the past year we worked with a system consisting of a $15^{\prime \prime}$ $x$ 15" multi-strip spark chamber connected to a memory plane of 256 ferrite cores. After an event occurred, the addresses of the flipped cores are recorded in sequence on paper tape by a coincidence half-current readout circuit. After testing several different patterns for the sense winding of the memory plane, we found one which gives a sufficiently large ratio between the signal of an addressed core reset by coincident half-currents and the signals induced by the noncoincident half-currents in the unaddressed cores. Thus, we now have a system for reading out the contents of the $25 \dot{6}$-core memory. 
Difficulty was encountered in achieving reliable performance in flipping the core threaded by the wire from the strip struck by the spark. Although there appeared to be more than adequate current available in a discharge to flip a core, the final state of the core was not always the one expected. We tried various schemes to dampen the oscillations that follow a discharge and may cause the state of a core to alternate. We also investigated the effects caused by the displacement current that accompanies the discharge. Before a satisfactory solution to the various technical problems had been achieved we decided to abandon this line of development in favor of new undertakings in $\mathrm{x}$-ray astronomy. (J. Stein, R. Chang, G. Clark)

\section{Low Energy Gamma Ray Spark Chamber}

Two processes have been suggested to explain the production of high energy cosmic $x$-rays. These are a) production of $\pi^{0}$-mesons in collisions between protons and interstellar gas, and b) electromagnetic processes, e.g., inverse Compton collisions of intergalactic electrons with low energy photons. The gamma ray spectrum from the decay of $\pi^{0}$-mesons has a peak at $70 \mathrm{MeV}$ independent of the $\pi^{0}$ spectrum; the spectrum that results from electromagnetic processes is monotonic. A measurement of the energy spectrum in the region 10 to $70 \mathrm{MeV}$ will distinguish between these two possibilities.

A balloon-borne instrument has been constructed for this purpose. It consists of a multiple gap spark chamber built with plates of thin aluminum foil. The chamber is triggered by an arrangement of scintillation counters and the photon energy estimated by pulse height analysis, multiple scattering, and the opening angles of the electron pairs.

In the fall of 1965 this experiment was transferred to the University of Wisconsin. A flight is expected in 1966. (P. Higbie, W. Kraushaar)

\section{Interplanetary P lasma}

\section{A. Satellite Instrumentation}

Flight instruments for EOGO B, P ioneer A and B and the A-IMP series of spacecraft are currently being prepared for launch. The EOGO system has been modified to improve its performance as much as possible; $P$ ioneer flight unit 1 was integrated into the spacecraft and successfully launched; the A-IMP prototype and first flight unit have been delivered for integration. (H. Bridge, A. Lazarus, and J. Davis, LNS; R. McMahon and J. McCarron, Lincoln. Lab. ; R. Baker, R. Butler, R. Lamson, and R. Rasche, J. Maxwell, Center for Space Research)

\section{B. Plasma Detector Testing}

- The proton acceleration source has been improved to the point of providing a stable beam with an energy spread of $\sim 50$ volts. D. C. testing on plasma cups showed unexpected currents due to production of secondary electrons from the energy determining grid but none of these 
currents should influence the measurements in space. (H. B ridge, J. Davis and A. Lazarus)

\section{Mariner 4 Results}

A report on preliminary analysis of the data was presented at the AGU meeting in Washington. The main features observed were:

1. The velocity of the solar wind fluctuates considerably from day to day. The values observed were never as great as those seen two years previously on Mariner 2.

2. The $\alpha$-particle to proton ratio also varies markedly though not much detailed analysis has been done as yet on this point.

3. No obvious effects were seen when the spacecraft passed into the earth's wake at $\sim 3000$ earth radii behind the earth.

4. Some signals were observed inside the magnetosphere as the spacecraft left the earth. A detailed study of the IMP -2 data will be necessary before it can be determined whether or not this phenomenon is a transitory effect. Further analysis is continuing.

(H. B ridge, J. Davis and A. Lazarus)

\section{E. Analysis of Plasma Measurements}

The analysis of the IMP -I data obtained by the MIT Faraday cup has been continued. The work outlined in the previous P rogress Report has been extended to include the later orbits of . the satellite. These orbits cover the portion of the transition region which lies on the dawn side of the magnetosphere (the sun -earth -satellite angle ranging typically between $40^{\circ}$ and $120^{\circ}$ ). As reported by A. Egidi, this portion exhibits a gradually increasing streaming of plasma protons with a directional distribution that is in agreement with a physical picture of a streaming fluid about a blunt body. Detailed numerical analysis with the computers has shown that one can achieve a good agreement between the calculated and the observed values of the proton fluxes in all five energy channels if one makes use of the empirical distribution function quoted in the previous P rogress Report. The agreement seems to be equally satisfactory for the energy spectra and the angular distribution of protons. However, best fitting has been obtained with surprisingly high densities (50-500 particles per c.c.) and low streaming velocities $(30-100 \mathrm{~km} / \mathrm{sec})$. The most probable "thermal" speeds seem to decrease very slowly as one approaches the dawn side of the transition region (dropping from $\sim 180$ to $\sim 100 \mathrm{~km} / \mathrm{sec}$ ). A cursory pre-publication com parison of the above results with those obtained by the Ames group with an electrostatic analyzer, although suggestive of possible inconsistencies between both detectors, is as yet inconclusive. A meaningful and unambiguous comparison can only be afforded after the electrostatic analyzer data have been subjected to the șame type of mathematical analysis as were those obtained by the Faraday cup. Such a parallel analysis is now being prepared for the computer program. The program will be kept sufficiently general so as to make it applicable to other similar detectors, such as that used by the Los Alamos group on the Vela satellite series. (G. Moreno, S. Olbert) 


\section{Deuterium Detector}

\section{A. Introduction}

Although there have been a number of attempts to detect deuterium in the solar atmosphere, using the isotope shift in the spectral lines of deuterium relative to ordinary hydrogen, it has not been detected. The observations were able to put an upper limit of between $4 \times 10^{-5}$ and $10^{-4}$ on the deuterium abundance relative to hydrogen.. This is significantly lower than the observed abundance of deuterium on the earth and in meteorites, where it is about $1.4 \times 10^{-4}$ relative to hydrogen. Indeed, one of the major problems in astrophysics is to determine the origin of the terrestrial (and meteoritic) deuterium.

Although deuterium is produced in thermonuclear reactions inside stars, it is rapidly burned up by further thermonuclear reactions. Thus, for example, its relative abundance in the solar core is only about $10^{-17}$. Burbidge and others have considered the possibility that flare activity on stars may produce deuterium (and also lithium, beryllium, and boron) through bom-bardment of the stellar surface material by high-energy particles accelerated during the flare. There is some indication that this may happen on the sun, although the experimental evidence is not conclusive. For example, spectroscopic observations of a solar flare on February 10, '1956, by Goldberg and co-workers indicated a possible deuterium concentration of 5 to $10 \%$ in the flare. However, this result was not confirmed by other observers.

Satellites and space probes now offer the possibility of obtaining information on the relative abundances of elements on the surface of the sun through direct analysis of the solar wind. This report describes an experiment to detect deuterium in the solar wind, and distinguish it from other nuclear species, which will be present in far higher abundance.

\section{B. The Experiment}

The principle of operation of the instrument is the following:

Solar-wind particles enter the instrument through an aperture several inches in diameter. A radial electric field accelerates and focuses the positive ions onto a titanium target containing about 1 curie of tritium absorbed in its surface. Nuclear reactions between incident deuterons and the tritium produce $3.6 \mathrm{MeV}$ alpha particles, which are detected by a solid-state detector placed close to the target. At a deuteron energy of $100 \mathrm{keV}$, the cross-section for the deuteriumtritium reaction is about 5 barns, which is many orders of magnitude larger than the cross-sections for other nuclear reactions that can occur between other components of the solar wind and the tritium. Also, since the alpha particles from the deuterium-tritium reaction are practically monoenergetic, they are easily distinguished from extraneous events that may be recorded by the solid-state detector.

C. Laboratory Tests

A prototype model of the deuterium detector has been tested, and the results of these tests indicate that the technique is feasible for satellites and space vehicles. 
A counting rate of about one alpha particle per minute would be observed for a relative deuterium abundance of $10^{-5}$ in the solar wind. In the tests, the instrument detected quite easily the deuterium present in ordinary laboratory hydrogen, where it has a relative abundance of about $1.5 \times 10^{-4}$. During operation of the detector over a period of a few months, no background or extraneous events were observed under ordinary laboratory conditions.

\section{Further Work}

1. Investigation of background.

We bel ieve that the background will be negligibly small in this experiment because the detector discriminates very strongly against false events. The results obtained so far have been encouraging in this respect, but further tests will be carried out this spring and summer:

(a) The detector will be exposed to a beam of low-energy $\mathrm{He}^{3}$ ions, to study the background due to nuclear reactions between $\mathrm{He}^{3}$, which may also be present in the solar wind, and the tritium in the target.

(b) The detector will be exposed to beams of energetic particles ( 1-20 MeV), to evaluate the background due to cosmic rays and energetic solar particles in space.

2. Engineering Development.

Further engineering development of the instrument is required to produce a prototype flight model. The electronic instrumentation will not present any significant problems, but the high voltage required for this experiment will make the design a difficult electrical and mechanical problem.

Indeed, the high-voltage supply will probably require the major part of the effort to develop a flight model. The compact high voltage supply that we have built for the laboratory instrument has shown that a unit can be built with the necessary mechanical strength for a space experiment, and an operating unit has been delivered as of February 1966 to the Space Center and is undergoing tests. The flight unit has been completed and is undergoing tests. (F. Scherb, now at University of Wisconsin; F. Floyd, MIT Space Center) (Work being performed as a joint experiment between MIT Space Center and the University of Wisconsin).

\section{Bolivian Air Shower Joint Experiment (BASJE)}

In December 1964 the enlarged detector array (20 density detectors over an area of $150 \mathrm{~m}$ radius) and the automatic fast timing apparatus were tested and found to be operating satisfactorily. It thus became possible (1) to record with reasonable efficiency Extensive Air Showers (EAS) up to a size of about $10^{8}$ particles $\left(\sim 2 \times 10^{17} \mathrm{eV}\right)$, and (2) to process the BASJE data without the 
Dr. Y. Toyoda left La Paz in February 1965, and Dr. K. Kamata arrive in May 1965. During the interim period, Dr. V. Domingo was the senior physicist with BASJE in La Paz. In June, Dr. Domingo arrived at M.I.T. for a two-month visit to complete his contribution to the BASJE. He is preparing a proposal, in conjunction with the Japanese group, which requests support for an emulsion spark chamber to be used in a detailed study of the EAS nuclear core at Mt. Chacaltaya.

Three papers were presented to the Ninth International Conference on Cosmic Rays in London (September 1965). (H. Bradt, G. Clark, M. LaPointe, A. Krieger, S. Rappaport and C. Forline)

\section{Composition of Primary Cosmic Rays}

The work in this area has been concentrated on determining the suitability of CsI(Tl) as a detector for distinguishing primaries by means of its fluorescence decay time characteristic. A. Womack completed his Master's thesis, concluding that the method was not suitable for distinguishing low $-z$ particles amid the high counting rate of primary protons. Nevertheless, the mechanism by which the decay time varies is of interest itself. Womack's thesis work provided data on $\mathrm{H}^{1}, \mathrm{H}^{2}, \mathrm{He}^{3}, \mathrm{He}^{4}, \mathrm{~B}^{11}, \mathrm{C}^{12}$, and $\mathrm{O}^{16}$ particles stopping in a crystal with energies up to $10 \mathrm{MeV} /$ nucleon. All these particles produce curves of effective fluorescent decay time vs $\ln \mathrm{E}$ that are straight lines and whose slopes and intercepts are characteristic of the stopping particles. Two other theses were done by seniors to investigate 1) if two particles of the same $\mathrm{dE} / \mathrm{dx}$ but different mass traverse a thin scintillator, are the decay times identical?, and 2) how does a variation in $\mathrm{Tl}$ content affect the decay time? The answer to the first question (D. Grimes), while not completely unambiguous, appeared to be negative. $7.5 \mathrm{MeV}$ protons and 12-15 MeV deuterons from the MIT Cyclotron were sent through an 11 mil CsI(Tl) crystal. Although the proton's dE/dx was not strictly constant, the light it produced seemed to have a significantly longer decay time than that from a $13.8 \mathrm{MeV}$ deuteron (which has the same average $\mathrm{dE} / \mathrm{dx}$ ).

The second investigation (D. Slevin) gave the surprising result that increasing $\mathrm{Tl}$ concentration increased the decay time of light produced by deuterons passing through the scintillator whereas the opposite phenomenon was observed for light from stopping $\alpha$-particles. In both cases, the total light produced increased with increasing $\mathrm{Tl}$ concentration.

An interesting feature common to both cesium and sodium iodide scintillators has been observed. We noticed that the total light output of CsI(Tl), though known to be non-linear with energy and to be dependent upon the type of incident charged particle, could be described by a linear function of $E_{0} \beta_{0} / Z^{2 / 3}$ where $E_{0}$ is the initial .energy of a nuclide which stops in the scin tillator, $Z$ its nuclear charge and $\beta_{0}$ its initial velocity. The relationship holds for ions with $\mathrm{Z}=5$ to 8 . P rotons show a marked deviation from this relationsh $\mathbf{p}$. The same relationship holds for scintillation light from $\mathrm{NaI}(\mathrm{Tl}) \mathrm{Z}=5$ to 10 . Although the mechanism responsible for this 
necessity of scanning projections of photographs of the fast timing oscilloscopes. With this arrangement the detector array has operated nearly continuously since January 1965 , with two basic trigger requirements: (1) a low-mu requirement of less than 4.0 particles recorded in the shielded $60 \mathrm{~m}^{2}$ detector array, and (2) a large shower requirement, which preferentially selects showers in the size interval of $10^{7}-10^{8}$ particles.

At the end of December of 1965, approximately 40,000 large showers and an equal number of low -mu showers had been accumulated. The majority of these showers have been processed at the University of Maryland and M.I.T. Analysis of the processed data indicates the continued existence of a class of mu-less EAS of relative frequency $5 \times 10^{-4}$ and with arrival directions that suggest an anisotropy in right ascension $w$ ith the maximum toward 200-220 degrees. Examination of the presently analyzed sample of about 15,000 large EAS indicates no evidence as yet for a special low-mu class of EAS in the region of $10^{17} \mathrm{eV}$. This places an upper limit upon the rate in intergalactic space of the gamma ray production mechanism:

$$
\mathrm{p}+\gamma_{\text {starlight }} \underset{\pi_{0} \rightarrow 2 \gamma}{\rightarrow} \pi_{0}+\mathrm{p}
$$

In turn, this places an upper limit upon the starlight and cosmic ray densities in intergalactic space. At present the upper limits derived from our data are comparable to or somewhat greater than the currently assumed densities. It is our hope that within a year the upper limit can be reduced to several times less than the assumed values. This result will be of significance because of our present nearly complete lack of knowledge about these quantities, and because of their relation to cosmological theories.

Mr. A. Krieger continues to build up the experimental apparatus for a measurement of the pool of Cerenkov light emitted by the electrons of an EAS during its traversal of the atmosphere. Thirteen detectors, each with about $900 \mathrm{~cm}^{2}$ of sensitive photocathode surface, will be positioned in a circular pattern of $600 \mathrm{~m}$ diameter on Mt. Chacaltaya. The data from these detectors in conjunction with that from the present array should yield results concerning shower development high in the atmosphere. In particular, it should be possible to select a group of showers that contains a high proportion of photons relative to electrons and therefore preferentially derive from heavy primaries. The thirteen detectors have been constructed, and the design of the circuitry is nearly complete. The equipment should be ready for operation in May 1966, and preliminary measurements will be taken in the summer of 1966 .

A Monte Carlo simulation of the nuclear core of an EAS has been performed upon an electronic computer at MIT. Simulations using Fe and proton primaries as well as différing models of nuclear interactions have been compared to the data from the $60 \mathrm{~m}^{2}$ shielded detector array. The coarseness of the spatial resolution of these fifteen $4 \mathrm{~m}^{2}$ detectors renders difficult the identification of showers arising from a given primary particle, though our early results indicate that in certain instances it may be possible to do so.

At MIT, we derived from the data an energy spectrum with improved statistics, and with fluctuations in the nuclear cascade taken into account. Dr. LaPointe is continuing th is work at the University of Maryland. 
relationship is not understood, it does seem to be related to the effective charge of the stopping particle (which is also proportional to $\beta / \mathrm{Z}^{2 / 3}$ ). The deviation of protons from this general relationship should provide an important clue to the nature of the scintillation mechanism. A more complete discussion will be published in the Physical Review.

We feel that further progress in understanding the nature of the scintillation process would require a significant commitment of time and financial resources. At, the present time we cannot make such a commitment and intend to carry on such work on a sporadic basis. (D. Grimes, A. Lazarus, S. Liu, D. Slevin and A. Womack)

\section{Theoretical Studies of Cosmic Rays as a Relativistic Gas}

The theoretical studies reported under the above heading in previous Progress Reports have been expanded to include a new feature in the structure of the galactic magnetic fields, the so-called "neutral sheets". One can present theoretically plausible arguments that a nearly antiparallel configuration of neighboring magnetic lines may be quite common in the equatorial portions of the galactic arms. If this be the case, there must exist confined regions where the magnetic field is extremely weak while the electric field may. well retain its large-scale typical strength. Such a situation introduces a new form of an acceleration mechanism provided there are a sufficient number of scattering irregularities in the medium. The energization of cosmic rays comes about essentially at the expense of the magnetic field energy that is destroyed within the neutral sheet by dissipative processes of the inflowing (partially ionized) galactic gas. Since the recent radio astronomical observations support the hypothesis of neutral sheets we have undertaken to analyze the behavior of cosmic rays under such conditions in a quantitative manner. The pertinent transport equations are per force more complex than those commonly used by others. Nevertheless, it is hoped that, at least for some idealized geometries, the problem can be pursued sufficiently far to insure definite conclusions concerning the importance of this phenomenon. (A. Klimas, S. Olbert)

\section{Infrared Astronomy}

A continuing program of infrared observations of selected regions of the sky is in progress at 1.61 and 2.15 microns. A sixty inch rhodium mirror of $f / 0.4$ is being used, and a limiting magnitude of $8.5 /(\min )^{2}$ has been achieved. (G. Garmire) 
A balloon-borne detector for the detection of solar neutrons and gamma rays was designed, and certain facets of its operation were tested by Mr. P. Oddone as his senior thesis project.

Another detector with a rough directional response is being tested by Mr. G. Schleickert, as his senior thesis project. However, at the present time, we do not have plans to carry out a major search for solar neutrions and gamma rays by means of balloon-borne detectors. (H. B radt, P. Oddone, G. Schleickert, and S. Rappaport)

\section{Time Variations of Cosmic Rays}

Continuous observations of cosmic ray meson intensities are made in the $\mathrm{P}$ enthouse of Building 26. The data obtained are being punched on IBM cards, tabulated and plotted automatically.

The analysis of the daily variation of meson intensity has revealed that proper temperature correction has to be applied before it can be compared with the neutron intensity from other stations. The data from Deep River neutron monitor have been subjected to Fourier analysis and the smoothed out daily variation built up from the first two harmonics. It is found that for the period Nov. 27, 1963, to Feb. 17, 1964, the time of maximum and time of minimum are nearly along the 1800 and 0900 directions respectively outside the influence of the earth's field. The time of minimum appearing along the 0900 direction coincides with the garden hose direction from the sun. These results have been published in Nature. Further analysis using the meson data from Deep River and meson and neutron data from a network of stations for an extended period is in progress.

The study of the short period variations using the data from the MIT meson monitor has shown that the short period variations are not prominent above the $0.2 \%$ level, which is barely above the statistical limit. Similar analysis using data from Canadian stations has confirmed the above results. Some theoretical investigation about this problem is underway and the results will be published soon as a paper.

A study of the relation between plasma parameters, geomagnetic activity and the cosmic ray intensity daily variation is also being planned using the data collected from the IMP satellites and the cosmic ray monitor. (G. L. Pai, M. Wada and V. Sarabhai)

\section{Supernova X-Rays}

The continuous spectrum of $\mathrm{x}$-rays from a couple to fifty kilovolts from the Crab Nebula, a supernova remnant of the year 1054, was described as the emission from hot plasma which gained heat from radioactivity or from internal shocks, and which could lose it mainly by 
radiation. As the plasma expands, the loss rate is reduced because of the lower density, since loss is via bremsstrahlung. Thus the plasma warms up, radiating faster at the higher temperature until a near steady-state sets in at $\mathrm{x}$-ray temperatures.

This theory was published in the Physical Review Letters, May 10, 1965. Experiment since then has shown a thermal spectrum, as predicted by the theory.

Work is in progress on a review article for the Annual Reviews of Astrophysics on $x$-ray sources. (P. Morrison, with L. Sartori of the Science Teaching Center, MIT)

\section{Supernova Visible Light at Times Close to Outburst}

The light curve and optical spectrum of the first two years after the outburst of a type I supernova are explained in some detail. Here radioactivity has no role to play at all. The key point is that the light is fluorescence, : mainly from HelI, induced by a quick burst of $u-v$ from the explosion. The slow regular visible decay is a kinematic consequence of this mechanism, taking a nearly uniform medium to surround the star. The fit to the observations given by this theory is excellent. Details of the spectrum are also plausibly explained.

Work will continue on other related phenomena, in other supernovae types and in quasars. (P. Morrison)

\section{P UB LICATIONS}

G. Clark, "Balloon Observation of the X-ray Spectrum of the Crab Nebula above $15 \mathrm{keV}$ ", Phys. Rev. Letters 14, 91 (Jan. 1965). G. Clark, "Positions of Three Cosmic X-ray Sources in Scorpio and Sagittarus", Nature 207,
584, (1965).

G. Garmire and W. L. Kraushaar, "High Energy Cosmic Gamma Rays", Space Sci. Rev. 4, 123 (Feb. 1965).

G. Garmire, "Intergalactic Photon Density", Ninth International Conference on Cosmic Rays, London (1965).

R. Giacconi, H. Gursky and J. Waters; G. Clark and B. Rossi, "Observation of Two Sources of Cosmic X-rays in Scorpius and Sagittarius", Nature 204, 981 (Dec. 1964).

W. L. Kraushaar, G. W. Clark, G. Garmire, H. Helmken, P. Higbie, and M. Agogino, "Explorer XI Experiment on Cosmic Gamma Rays", Astrophys. J. 141, 845 (April 1965).

M. Oda, G. Clark, G. Garmire, and M. Wada; R. Giacconi, H. Gursky, and J. Waters, "Angular Sizes of the X-ray Sources in Scorpio and Sagittarius", Nature 205, 554 (Feb. 1965).

V. Sarabhai, G. L. Pai, and M. Wada, "Anisotropy of Galactic Cosmic'Rays and the Interplanetary Magnetic Field", Nature 206, 703 (May 1965).

\section{ADDRESSES AND INVITED P AP ERS}

H. Bradt, "Extensive Air Showers at Mountain Altitudes: A Search for High Energy Cosmic Gamma Rays", invited paper presented at the Summer meeting of the American Physical Society in New York? June.25, 1965). 
H. Bradt, "The Bolivian Extensive Air Shower Experiment"' MIT Physics Colloquium, (Nov. 18, 1965)

H. Bradt, "A Monte Carlo Simulation of the Nuclear Active Cascade in EAS", Paper EAS-11 at Ninth International Conference on Cosmic Rays, Longon (September 1965).

H. Bradt, G. Clark, M. LaPointe, "The Primary Energy Spectrum from $8 \times 10^{14} \mathrm{eV}$ to $4 \times 10^{17} \mathrm{eV}$ ", Paper EAS-31 at Ninth International Conference on Cosmic Rays, London (1965).

A. Lazarus, H. B ridge, (with C. Snyder, 'JP' L), "P lasma P robe Observations from Mariner 4". A.G.U. 46thi Annual Meeting, Washington (April 19, 1965).

G. Clark, "Observational Work on Cosmic Gamma Rays", Second Texas Symposium on Relativis - · tic Astrophysics, (November 1964).

G. Clark, "Observations of Cosmic Gamma Rays", American Physical Society, Berkeley Meeting (Dec. 1964).

G. Clark, "X-ray and Gamma Ray Astronomy", Columbia University Physics Colloquium, (Feb. 1965).

G. Clark, "Recent Observations of X-Ray Sources", Royal Astronomical Society and the Royal Irish Academy, Dublin (Sept. 6-8, 1965).

G. Clark, "Observations of Cosmic X-Rays from Balloons", 9th International Conference on Cosmic Rays, London (Sept. 1965).

G. Garmire, "Intergalactic Photon Density", 9th International Conference on Cosmic Rays, London (Sept. 1965).

M. Oda, "X-Ray and Gamma-Ray Astronomy", 9th International Conference on Cosmic Rays, London (Sept. 1965).

S. Olbert, "Interpretive Analysis of the MIT Solar Wind Data", invited paper at Ames-NASA, Stanford University Conference on Shock Waves in Collision-free P lasmas at - Moffett Field, California (March 3, 1965).

S. Olbert, "Solar Wind - Recent Experimental Results and their Interpretation", invited talk at Cornell University, Department of Astronomy (March 19, 1965).

S. Olbert, "Solar Wind and its Interaction with the Magnetosphere" Invited paper, Am. Phys. Soc., Central States Meeting at Chicago (Oct. 29, 1965. Invited paper, Physics Colloquium, University of Wisconsin (Nov. 1, 1965).

B. Rossi, "Cosmic Ray Particles of Highest Energy" Invited paper at the Texas Symposium on Relativistic Astrophysics, Austin, Texas (Dec. 1964). Physics Colloquium, B randeis, Waltham, Mass. (April 1965).

B . Rossi, six lectures on "The P roperties of Collisionless Plasma with Application to the Solar Wind and to Geomagnetically Trapped Particles", Scuola di Perfezionamento di Fisica, University of Rome (May 1965).

B. Rossi, "X-Ray Astronomy", Haverford Colloquium, Haverford College, Pa. (October 1965).

F. Scherb, "The Solar Winds", Colloquium talk at the University of Wisconsin, (March 26, 1965).

\section{THESES}

R. P. H. Chang, "The Effect of Space Charge on Solar Wind Protons", B.S. (June 1965).

Mary Ellen Coffey, "Effects of Radiation on the Lifetime of Silicon Solar Cells", B.S. (May 1965)

J. L. Elliot, "A Stellar Interferometer for Faint Astronomical Objects", M.S. (May 1965).

C. W. Forline, "The P rimary Energy Spectrom of Cosmic Rays from $2 \times 10^{14} \mathrm{eV}$ to $4 \times 10^{16} \mathrm{eV}^{\text {" }}$ B.S. (June 1965).

D. Grimes, "An Investigation of the Dependence on Specific Energy Loss of the Decay Time of Scintillations in CsI(TI) excited by Charged Particles," B.S. (June 1965).

G. Herzlinger,"Diffraction Patterns from Lunar Occultations", B.S. (June 1965).

H. Harounian, "Propagation of Low Frequency P lasma Waves in the Solar Wind", B.S. (June 1965).

S. J. Hynek, "Cosmic Ray Observations on a Multi-Gap, Glass-Metal Shower Spark Chamber", B.S. (May 1965).

Karen A. Kolling, "A System for Measuring 8 to 14 Micron Radiation Flux from Celestial Objects", B.S. (June 1965).

C. Leong, "Search for the Anisotropy of Cosmic Rays", B.S. (Jan. 1965). 
P. G. Oddone, "The Detection of Solar Cosmic Ray Neutrons and Gamma Rays", B.S. (June 1965).

L. D. Pressman, "Studies of Stellar Scintillation and Atmospheric Turbulence", B.S. (June 1965).

D. Slevin, "The Effect of Thallium Concentration on the Fluorescent Response of CsI(TI)", B. S. (June 1965)

A. S. Tanenbaum, "A Method for Determining the Relative Positions of Two Celestial Radiation Sources"; B.S. (May 1965)

A. Womack, Jr., "A Study of the Fluorescent Decay of CsI(Tl) as a Means of Distinguishing Charged Particles", M.S. (June 1965).

K. B. W. Yip, "An Investigation of Infrared Radiations from the Andromeda Nebula", B.S. (June 1965). 


\section{ACCELERATOR P HYSICS COLLABORATION}

During this period we completed the exposure at BNL to extend our study of $\pi^{-}$interactions to $4 \mathrm{BeV} / \mathrm{c}$.

Our previous experiment, from $600 \mathrm{MeV} / \mathrm{c}$ to $1200 \mathrm{MeV} / \mathrm{c}$, studied the reaction $\pi^{-}+\mathrm{p} \rightarrow$ $\mathrm{N}+$ neutrals.

This experiment extends this to $4 \mathrm{BeV} / \mathrm{c}$. The exposure was taken in our high-z spark chamber at the A.G.S. at B rookhaven National Laboratory, and analysis has been starled.

We also have proposed an experiment at the Cosmotron at BNL to study the branching ratios of the $\eta^{0}, \rho^{0}$, and $\omega^{0}$. This experiment should start in the summer of 1966 .

The photoproduction work at C.E.A. in the $12^{\prime \prime} \mathrm{H}_{2}$ chamber is continuing. A number of papers have been published (see publication list) and the final work should be completed by the summer of 1966 .

PEPR has progressed to the point where several events have been processed by the complete system. We will attempt to analyze 150,000 events of $\bar{p} p$ interactions in the next year. Production should start in the early summer of 1966.

P rogress on the $40^{\prime \prime}$ hydrogen chamber has been delayed by the accident at the C.E.A. A detailed examination of the chamber has indicated that it will be relatively inexpensive to repair. Plans to install the 40" chamber at Argonne National Laboratory are being investigated.

\section{P UB LICATIONS}

P. Bastien, T. Watts, R. Yamamoto, M. Alston, A. H. Rosenfeld, F. Solmitz, H. Taft, "P rogramming for the PEPR System", Methods in Computational Physics, 5 (Feb. 1966).

B. Brabson, L. Rosenson, et al. , "Improvements to a Spark Chamber System for High Energy $\gamma$-Ray Detection", Rev. Sci. Instr. 37, 118 (January 1966).

G. K. Karageorge, "Investigation of 'Scotchlite' as a Reflective Surface in a Liquid Hydrogen Bubble Chamber", Rev. Sci. Instr. 36, No. 6 (June 1965).

I. A. Pless, L. Rosenson, G. Salandin, "Spark chamber System for High Energy Gamma Ray Detection", Rev. Sci. Instr. 35, 1642 (1964)

I. A. Pless, "P EPR System", I. E.E.E. Transactions on Nuclear Science, (August 1965).

\section{CONFERENCES AND MEETTNGS}

\section{Abstracts}

Cambridge Bubble Chamber Group, "Photon Interactions in a Hydrogen Bubble Chamber in the Energy Range 0.5 - 5.9 BeV", DESY Conference, Hamburg, Germany, June 1965:

A. M. Shapiro (B rown), "Cross Sections and Particle Distributions"

B. T. Feld, (MIT), "Strange Particle Production"

M. E. Law (Harvard), "Vector Meson Production"

Y. Eisenberg (Weizmann), "Nucleon Isobar Production"

L. Guerriero (Padova), "Multipion P roduction and other Complex Reactions" 
Addresses and Invited P apers

I. A. Pless, "P.roduction of $\mathrm{N}^{* *}$ and Eta-mesons", Erevan Summer School, Erevan, U.S.S.R., (May 1965), to be published.

I. A. Pless, "Automatic Scanning and Measuring of Spark and Bubble Chamber Events", Erevan Summer School, Erevan, U.S.S.R., (May 1965), to be published.

I. A. Pless., "P.EPR System", Purdue Conference on Instrumentation for High Energy Physics, Purdue University, (May 1965).

\section{THESES}

W. J. Podolsky, "A Search for the $E^{0}$ and other Scalar Meson Resonance", B.S. (June 1965).

A. H. Rogers, Jr., "P roduction of Rho-Mesons by High Energy Photons in Hydrogen", Ph. D. (June 1965).

C. W. Rogers,. "Analysis of $\mathrm{N}^{*}(3 / 2,3 / 2)$ P roduction as seen in $(\gamma, \rho)$ Interactions", Ph. D. (June 1965).

C. Schneider, "Corrections of the Energy and Spark Chamber Cascade Properties of Gamma Rays", M.S. (May 1965). 
During this period, analysis was begun on data collected on the angular distribution of photoneutrons from Beryllium. Programs have been written for the LNS IBM 7044 computer to extract cross sections and angular distribution parameters from the data.

The data obtained with a $5.3 \mathrm{MeV}$ electron beam was analyzed with the computer programs. A fore-aft asymmetry, in this case backward peaking, in the angular distribution of the photoneutrons was found in the region of excitation of $\mathrm{Be}^{9}$ from 3.2 to $4.6 \mathrm{MeV}$. This confirms the preliminary results described in the last progress report. The data have not been corrected for target scattering, which would tend to decrease the asymmetry. The corrected results there fore show an even greater fore-aft asymmetry. An asymmetry can occur only when there is an interference between neutron production amplitudes from states of different parity. A fore-aft asymmetry is therefore interpreted as evidence for the existence of an odd parity level in the region of excitation of $\mathrm{Be}^{9}$ from 3.2 to $4.6 \mathrm{MeV}$.

The remaining data taken at electron beam energies from $5 \mathrm{MeV}$ to $12 \mathrm{MeV}$ in steps of $1.5 \mathrm{MeV}$ at angles of $25^{\circ}, 76^{\circ}, 155^{\circ}$ is being analyzed. On completion, this analysis will yield photoproduction cross-sections and angular distributions of photoneutrons for excitations leaving the $\mathrm{Be}^{8}$ daughter nucleus in its ground state and also in excited states accessible with these energies. (W. Bertozzi, S. Kowalski, T. Phillips, C. P. Sargent, and W. Turchinetz)

\section{Photoneutron Systematics}

The energy spectra of photoneutrons from $\mathrm{Bi}, \mathrm{Pb}^{208}, \mathrm{~Pb}^{207}, \mathrm{~Pb}^{206}, \mathrm{Tl}, \mathrm{Hg}, \mathrm{Au}, \mathrm{W}, \mathrm{Ta}, \mathrm{Er}$, Ho, Sm, Pr, La, I, Sn, and In were measured at $24^{\circ}, 75^{\circ}$, and $156^{\circ}$ using the MIT Linear Accelerator with 10 meter flight paths and $8 \mathrm{~ns}$ resolution. The bremsstrahlung endpoints used were $15 \mathrm{MeV}$ and $14 \mathrm{MeV}$. Subtracting the $14 \mathrm{MeV}$ runs (properly normalized), from the $15 \mathrm{MeV}$ runs yielded neutron spectra resulting from an equivalent photon spectrum peaked at $14 \mathrm{MeV}$ with a FWHM of $2 \mathrm{MeV}$.

The angular distributions of these, difference spectra are isotropic at low neutron energies. At higher energies they are given by the form

$$
\mathrm{W}(\theta)=\mathrm{a}_{0}+\mathrm{a}_{2} \mathrm{P}_{2}(\cos \theta) \quad \mathrm{a}_{1}=0
$$

The anisotropy $-\mathrm{a}_{2} / \mathrm{a}_{0}$ begins to increase at about $2-3 \mathrm{MeV}$ and reaches a maximum listed in Table 6.I. The anisotropy reaches a value of one-half of the maximum value at $31 / 2-41 / 2 \mathrm{MeV}$. 
Table 3.I

Preliminary Results

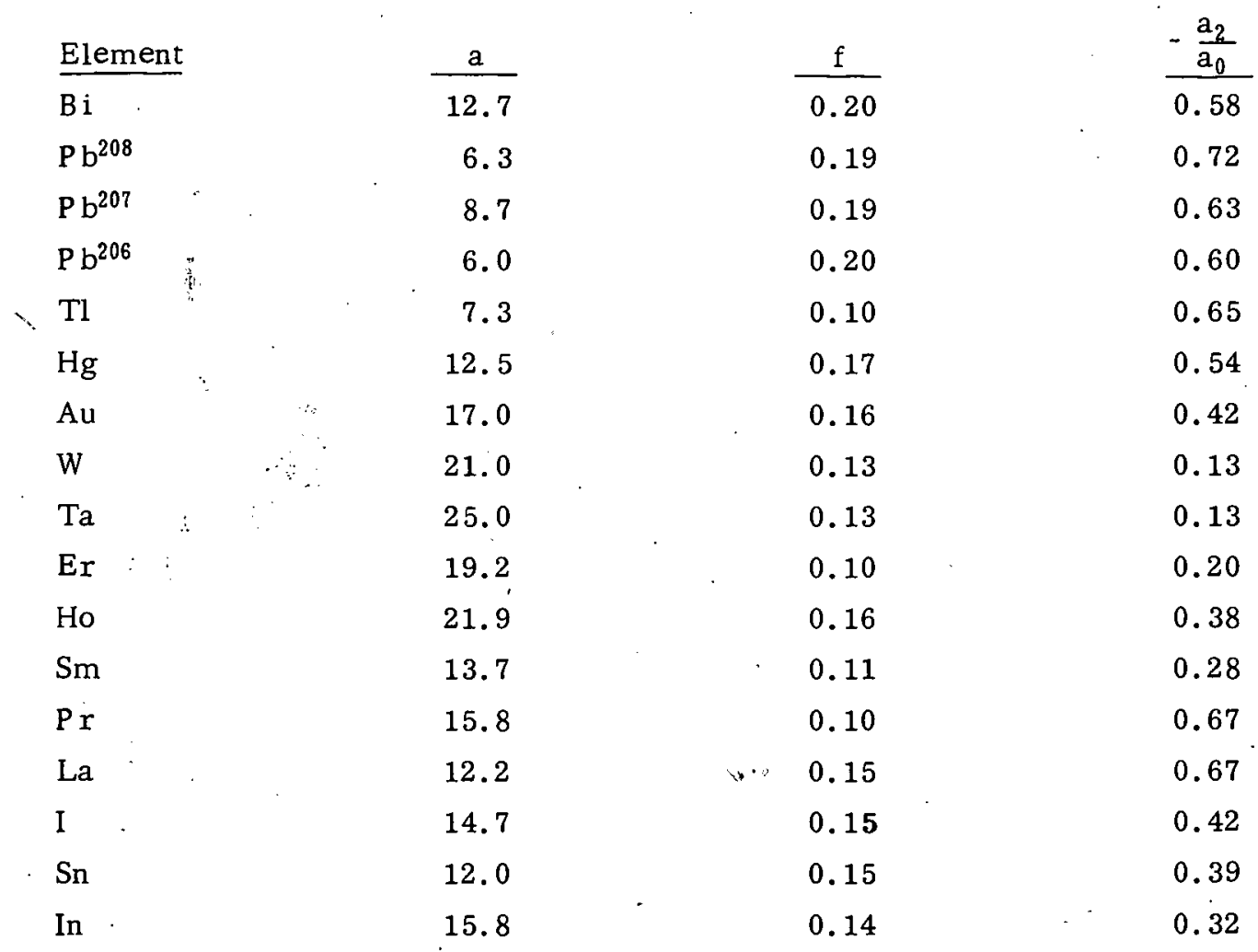

At low neutron energies, the difference spectra were fitted by an evaporation spectrum of the form

$$
\frac{d N}{d E_{n}} \propto E_{n} \sigma_{c} \frac{e^{2 \sqrt{a U}}}{(U+t)^{2}}
$$

$U=E^{*}-\delta p$, the excitation energy of the residual nucleus minus the energy required to break a neutron or.proton pair if $\mathrm{N}$ or $\mathrm{Z}$ is even.

$\sigma_{c}=$ the inverse capture cross section, which is estimated from optical model calculations. $\mathrm{a}=$ the Fermi gas level density parameter, which is determined by a least squares fit to the data between 1.0 and $3.0 \mathrm{MeV}$.

$t=$ the nuclear temperature defined by $U=a t^{2}-t$.

All of the data fit this curve well up to approximately $3 \mathrm{MeV}$ neutron energy, at which point the spectra exhibited an abrupt change in slope and an excess of high energy neutrons. This abrupt change in slope corresponds to the same energy as the midpoint of the angular distribution curves.

Therefore the data is characterized by an isotropic evaporation component dominating at low neutron energies, and a direct component with a strong angular distribution dominating at high neutron energies. The fraction $f$ of direct neutrons divided by total neutrons was calculated 
by subtracting the evaporation neutrons from the total spectrum.

Table 6. I lists the elements used, the Fermi gas level density parameter, a, the fraction, $f$, and the maximum value of $-a_{2} / a_{0}$ measured in this experiment. (W. Bertozzi, S. Kowalski, G. Mutchler, C. P. Sargent, W. Turchinetz)

\section{P ola rimeter}

For the past few months we have been making extensive tests on scattering corrections to data taken with the polarimeter. The instrument is a helium-xenon gaseous scintillation counter described in earlier reports. The tests have been made with a beam of $14.7 \mathrm{MeV}$ neutrons from the $\mathrm{t}(\mathrm{d}, \mathrm{n}) \mathrm{He}^{4}$ reaction using a Cockcroft-Walton owned by the Nuclear Engineering Department.

The first result of these tests was a correction to the calibration of the gas counter. The $\mathrm{Po}^{210}$ alpha sources gave a pulse height about $5 \%$ low for a $5.3 \mathrm{MeV} \mathrm{He}$ recoil. This is a pressure effect, being only $3 \%$ for a gas counter pressure of $400 \mathrm{psi}$ and $5 \%$ at $800 \mathrm{psi}$. The polarimeter runs of last year are being re-analyzed using this calibration correction.

The other results of these tests concern the scattering corrections to date and are obtained in the form of polarimeter response to a $14.7 \mathrm{MeV}$ neutron beam. The observed spectra are compared with computer calculations and provide a check on the theory of the instrument. With this check we expect to calculate the corrections to better than $50 \%$, which will significantly reduce the error bars on our data on deuterium. At present, uncertainty in these corrections has as large an effect on error bars as counting statistics.

The work on scattering corrections is planned to be finished by the end of summer, as is data analysis. (W. Bertozzi, F. Hanser, S. Kowalski, R. Rudzinski, C. P. Sargent, W: Turchinetz)

\section{Collaboration with the University of Saskatchewan (Canada)}

$\underline{\mathrm{Li}^{6}}(\gamma, \mathrm{np})$

The $\operatorname{Li}^{6}(\gamma, \mathrm{np})$ experiment was set up at the Saskatchewan Linear Accelerator. The six teen plastic scintillator proton detectors were mounted in an array covering from $50^{\circ}$ to $150^{\circ}$, with ten counters in the reaction plane and the remainder out of the plane. The four neutron detectors (the liquid scintillation counters used in MIT Linac time of flight work) were placed at $30^{\circ}, 50^{\circ}, 70^{\circ}$, and $90^{\circ}--$ angles determined by the target area and shielding geometry.

The electron beam used for this experiment was $140 \mathrm{MeV}, 600$ pulses $/ \mathrm{sec}, 1 \mu \mathrm{sec}$ pulse width, with peak currents between $1 \mathrm{~mA}$ and $20 \mathrm{~mA}$. The bremsstrahlung target was located at the end of the accelerator, inside the machine vault. The gamma-ray beam was carried through a series of lead collimators surrounded by lead,iron, and concrete shielding, producing a spot about 2 inches in diameter at the $(\gamma, \mathrm{np})$ target 75 feet from the bremsstrahlung target. The beam 
is buried in a lead brick $91 / 2$ feet further downstream; the beam dump is enclosed by about a three foot cube of iron. 'Two beam monitors were used: a scintillation counter in the gamma-ray beam to monitor the peak current and pulse shape, and a thin ionization chamber to measure the average current, which also was integrated.

The first approximately 50 hours of beam time were spent in improving the collimation and shielding -- reducing the beam -associated background seen by the proton counters -- and studying the spectra from $\mathrm{CD}_{2}, \mathrm{CH}_{2}$, and $\mathrm{Li}^{6}$ targets, trying to distinguish protons from electrons. A one-half inch thick plastic scintillator was placed in front of the five inch thick " $E$ " counter to serve as a "dE/dx" counter. By using a signal from the thin counter to drive the logic circuitry, with a threshold set at about $10 \mathrm{MeV}$ energy loss, it was possible to reduce the electron back ground considerably.

When the proton spectra were reasonably well understood and seemed to be consistent with expectations (comparing, for example, the $\mathrm{CD}_{2}$ results with the angular distributions quoted by Partovi (Ann. Phys. 27, 79, 1964) for the deuteron and me asured by Whitehead, et al., (Phys. Rev. 110, 941, 1958)) for carbon, we decided to try the coincidence experiment. The first runs (about 8 hours' worth) were made with eight " $E$ " counters alone, with four of them set at the proper angles to observe coincidences from the deuteron with the four neutron counters, for photons between 90 and $130 \mathrm{MeV}$. The time of flight scale for a given pair of counters was calibrated by attaching light pulsers driven from a common source to each counter. An additional timing check is provided by observing coincidences between the two $0.5 \mathrm{MeV}$ gamma-rays from $\mathrm{Na}^{22}$ with a nel tron counter and a proton counter at $180^{\circ}$. The proton pulse height scale was . established also with the light pulser, calibrated against the $\mathrm{Na}^{22} 1 \mathrm{MeV}$ Compton edge.

Neutron -proton coincidences from the deuteron, using the $\mathrm{CD}_{2}$ target, were easily detected. Coincidences from $\mathrm{Li}^{6}$ were also observed, centered about the deuteron-correlated angles, but the accidental rate was unfortunately high. In view of this, "dE/dx" counters were installed in front of all " $E$ " counters, with two aims -- to remove the electron contribution to the accidentals in the coincidence experiment and also to allow us to obtain believable proton singles spectra at the same time.

The 4096 -channel analyzer used for recording the singles data was operated with three parameters: eight counter, 16 channels of " $\mathrm{dE} / \mathrm{dx}$ " pulse height, and 32 channels of " $E$ " pulse height. The coincidence analyzer recorded the "E" pulse height only. When the singles spectra were displayed in this way, it was observed that a large part of the background was due to protons not from the target, passing through to the " $\mathrm{dE} / \mathrm{dx}$ " counter only. Accordingly, the accidentals were further reduced by requiring a $\mathrm{dE} / \mathrm{dx}$ - E coincidence to drive the logic.

Only a few hours of data were obtained with this new system before the accelerator had to be shut down. At this time the coincidence experiment looks quite favorable, and analysis of the singles data is in progress. We hope to get the 50 to 100 hours of beam time necessary to finish the experiment sometime within the next few months. ' $W$. Bertozzi, S. Kowalski, J. Matthews, C. P. Sargent, and W. Turchinetz) 
"W. Bertozzi, "Spectra and Polarization of Photoneutrons", University of Rochester, October 4, 1965.

\section{PUB LICATIONS}

\section{Abstracts}

G. S. Mutchler, W. Bertozzi, S. Kowalski, C. P. Sargent, W. Turchinetz, "P hotoneutron Spectra from Heavy Elements", Bull: Am. Phys. Soc. 10, 542 (1965).

T. W. Phillips, W. Bertozzi, S. Kowalski, C. P. Sargent, W. Turchinetz, "P hotoneutron Cross Sections and Angular Distributions from Be $e^{9 ",}$ Bull. Am. Phys. Soc. 10, 541 (1965).

$\underline{\text { Papers }}$

W. Bertozzi, P. T. Demos, S. Kowalski, F. R. Paolini, C. 'P : Sargent, W. Turchinetz, "Photoneutron Spectrometry by Tirne of Flight", Nucl. Instr. and Methods, 33, 199 (1965).

C. P. Sargent, W. Bertozzi, P. T. Demos, J. L: Matthews, and W. Turchinetz, "P rompt Neutrons from Thorium Photofission", Phys. Rev. 137, 1B, B 89 (1965).

\section{THESES}

D. Trevvett, "Threshold Photoneutrons from Barium and Lanthanum", B.S.

R. Rudzinski, "Measurement of Response Function of a Neutron Polarimeter Spectrometer", B.S.

G. S. Mutchler, "The Angular Distributions and Energy Spectra of Photoneutrons from Heavy Elements", Ph.D. 
exchange processes, as well as scattering, a minimum is seen at $\mathrm{t} \sim 0.7(\mathrm{BeV} / \mathrm{c})^{2}$ with a subsequent maximum at $\mathrm{t} \sim 1.2(\mathrm{BeV} / \mathrm{c})^{2}$. It is of some interest to see whether the same situation obtains for photoproduction; apparently, at this energy, it does not.

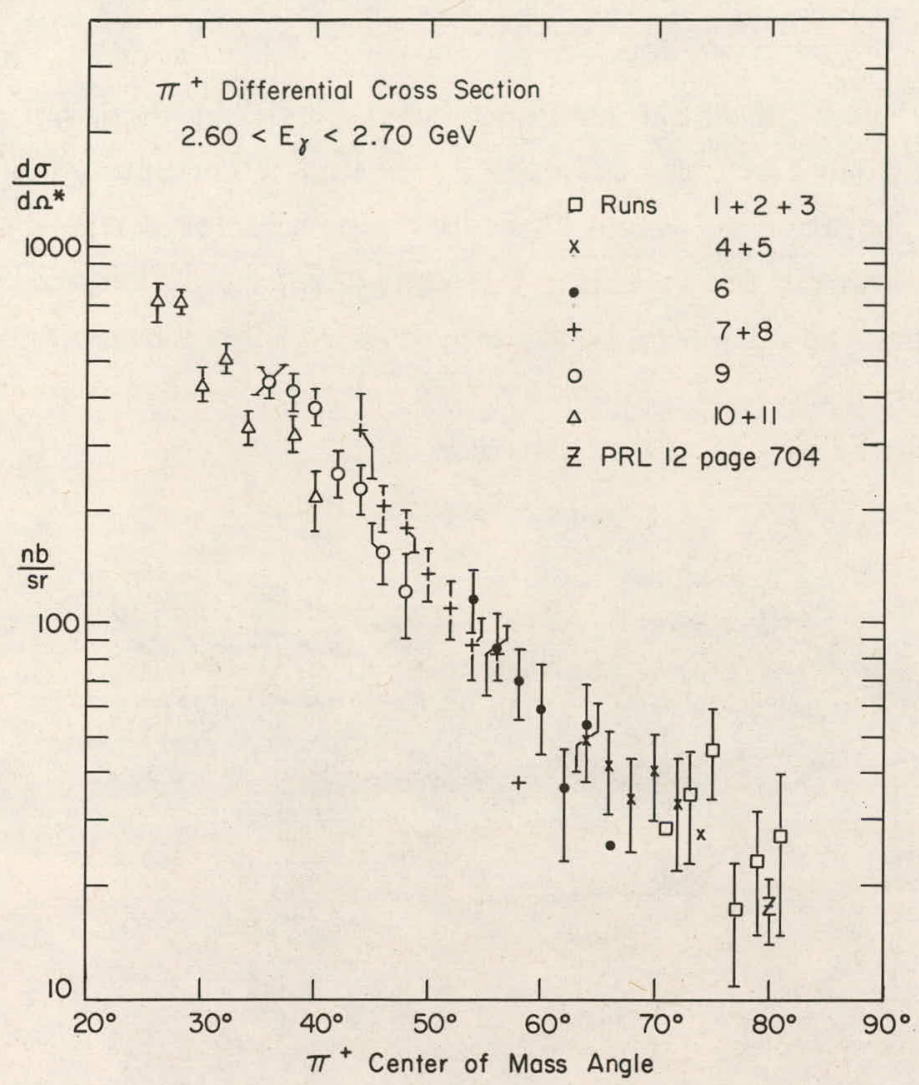

Figure 4.2

Photoproduction of $\pi^{+}$Mesons

The $\mathrm{K}^{+}$experiments measured the following reactions (see Fig. 4.3):
a) $\gamma+\mathrm{p} \rightarrow \mathrm{K}^{+}+\Lambda^{0}$
b) $\rightarrow \mathrm{K}^{+}+\Sigma^{0}$
c) $\quad \rightarrow \pi^{+}+\mathrm{n}$

This was done for $\mathrm{k}=3.7 \mathrm{BeV}$ and $\theta$ (center-of-mass) $=45^{\circ}$. This energy was picked to be at our previously discovered resonance. From SU(3) one predicts that an 8 resonance decays so that, for the matrix elements of processes $b$ and $c$,

$$
\mathrm{M}_{\mathrm{b}}=\mathrm{M}_{\mathrm{c}}
$$

We note with satisfaction that the cross sections are indeed equal within statistics. A further SU(3) prediction gives

$$
\mathrm{Mc}=-\frac{1}{2} \mathrm{Mb}-\frac{\sqrt{3}}{2} \mathrm{Mq}
$$

This gives rise to a triangular inequality between the three cross sections which clearly holds in view of the comparable cross sections. We have pursued these measurements with further runs at smaller angles and over a greater energy spread. 


\section{HIGH ENERGY ACCELERATOR PHYSICS GROUP}

I. Photoproduction Work

In the past period we finished the installation of the "tail" on the large spectrometer (Moby Dick) at the Cambridge Electron Accelerator; we finished mounting the counters and brought the system into operation in March. By deflecting particles vertically we can make use of the larger angular aperture of the system in the plane perpendicular to the deflecting plane, i.e., the horizontal plane, and thus get a better bite at an angular distribution in one setting $\left(7^{\circ}\right)$. Furthermore, by decoupling the $\theta$ and momentum measurement we obtain a better accuracy in calculating the photon energy by a factor $\sqrt{2}$ (see Fig. 4.1).

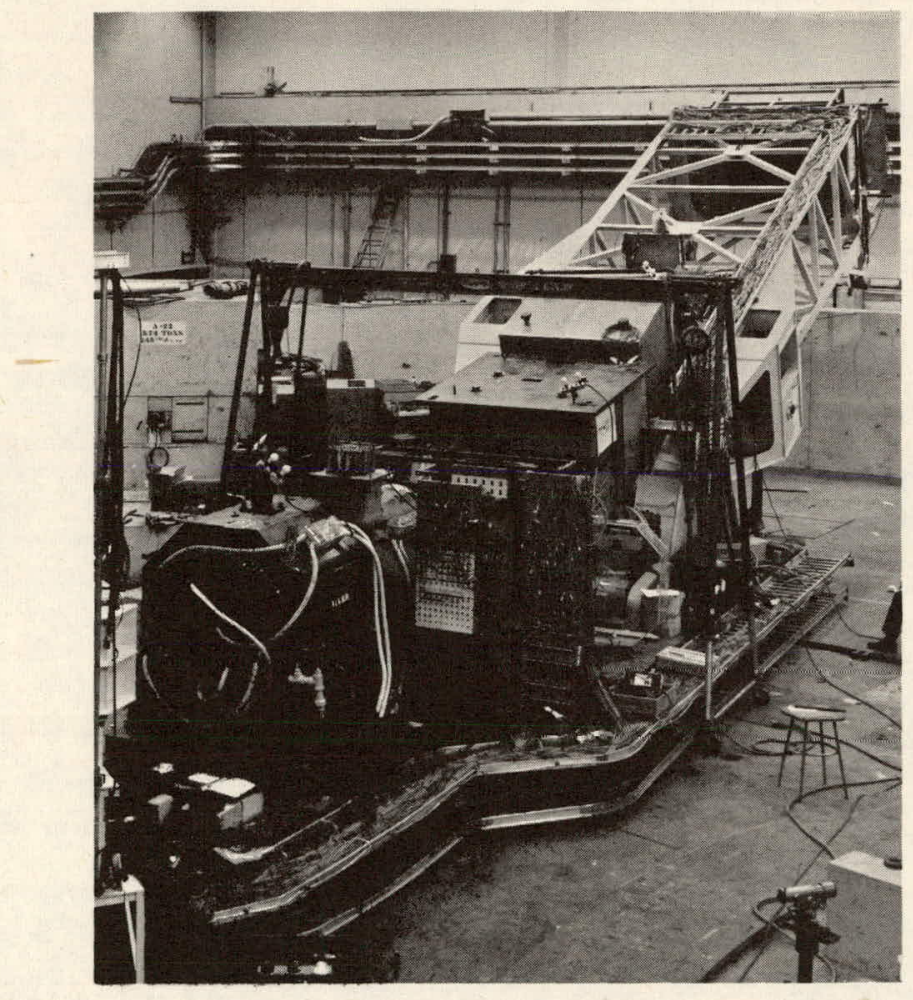

Figure 4.1

Photograph of Spectrometer

We also made improvements in the gas Cherenkov counter optical system so that it can detect a larger spread of velocities; this was needed to measure $\mathrm{K}^{+}$photoproduction efficiently.

During the spring we had runs on $\pi^{0}$ photoproduction with $\pi^{+}$photoproduction as a by -product, and on $\mathrm{K}^{+}$and $\pi^{+}$photoproduction. The $\pi^{0}$ photoproduction data are still being analyzed. The by product results on $\pi^{+}$photoproduction are shown in Figure 4.2. It shows the expected drop-off with momentum transfer that is also obtained in $\pi$-nucleon scattering. However, in charge- 


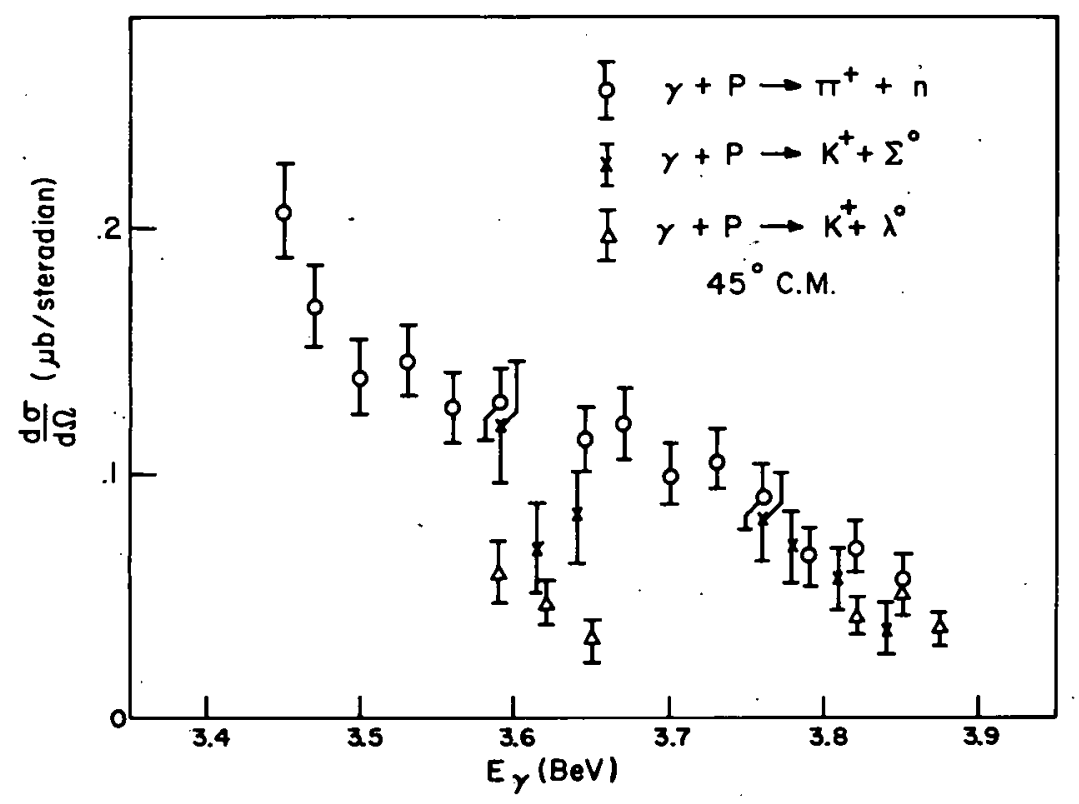

CENTER OF MASS DIFFERENTIAL ĆROSS SECTION.

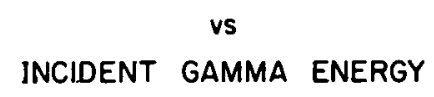

Figure 4.3

Photoproduction of $\pi^{+}$and $\mathrm{K}^{+}$Mesons

We are continuing with the installation of the on-line connection of the IBM 7044 to our Linc computer. We should have this system operating by fall.

The wire spark chamber, a prototype of a general system to be placed throughout our spectrometer is operating although the gap efficiency is not as high as desirable. We are working on this. (R. Alvarez, R. Fessel, V. Elings, D. Garelick, S. Homma, R. Lewis, W. Lobar, D. Luckey, L. Osborne, J. Uglum, Z: Bar-Yam, W. Kern)

\section{Large Angle $\pi^{+}$and $\mathrm{K}^{+}$Photoproduction}

Work is continuing on the construction and testing of equipment. The steel platform upon which the spectrometer and detecting apparatus is to be mounted has been designed and is on order. Wide-gap spark chambers and the associated high voltage pulsing appratus have been built and tested. The scintillation counters are in the final stages of construction. Electronics for the fast triggering logic have been assembled and tested. A narrow gap, aluminum plate spark chamber for separating $\pi^{+}$and $\mathrm{K}^{+}$mesons, by virtue of their differing ranges, is being built commercially and should be delivered in the near future.

The major remaining tasks, in addition to final alignment of the detecting system, are construction of a hydrogen target cell and the optical system for the front spark chambers.

(R. Alvarez, G. Cooperstein) 


\section{Spark Chamber Work}

A. $\pi^{-}+p \rightarrow \pi^{0}+n$. Charge Exchange Scattering at High Energies

The differential and total cross sections for elastic charge -exchange scattering $\pi^{-}+\mathrm{p}^{\rightarrow} \rightarrow \pi^{0}+\mathrm{n}$ has been measured at incident laboratory pion momenta of $6,8,10,12,14,16$, and $18 \mathrm{GeV} / \mathrm{c}^{1}{ }^{1,2}$

The differential cross sections at 6 through $16 \mathrm{GeV} / \mathrm{c}$ are shown in Figure 4.4. The main features of the differential cross sections are the same at all incident momenta over the range of momentum transfer measured: $0 \geqslant t \geqslant-0.5(\mathrm{GeV} / \mathrm{c})^{2}$. The region between $\mathrm{t}=0$ and about $\mathrm{t}=-0.1$ $(\mathrm{GeV} / \mathrm{c})^{2}$ is rather flat, although there appears to be a slight dip near $t=0$, which persists when the data are plotted in intervals with half the width of those used in Figure 4.4. Beyond $t=-0.1$ $(\mathrm{GeV} / \mathrm{c})^{2}$, there is an approximately exponential decrease, with slopes which closely approximate those observed in the elastic scattering of $\pi^{-} \mathrm{p}$ and $\pi^{+} \mathrm{p} .^{3}$

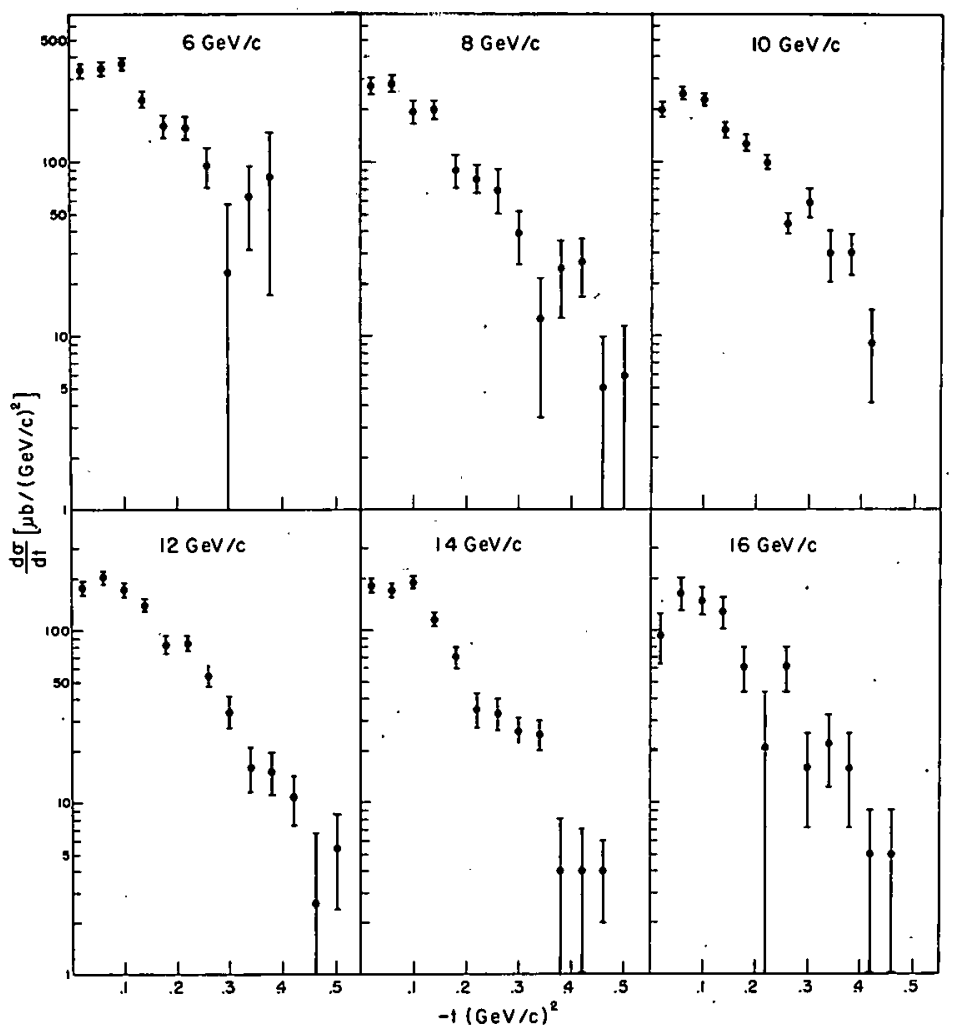

. Figure 4. 4

The differential $\pi^{-}+\mathrm{p} \rightarrow \pi^{0}+\mathrm{n}$ charge -exchange cross sections at $6,8,10,12,14$, and $16 \mathrm{GeV} / \mathrm{c}$. The errors shown are statistical only. The systematic uncertainties are $\pm 8 \%$.

Since $d \sigma / d t$ is flat within statistics from $t=0$ to about $t=-0.12(\mathrm{GeV} / \mathrm{c})^{2}$, we approximated $(\mathrm{d} \sigma / \mathrm{dt})_{\mathrm{t}=0}$ by the average value over this region. For all $\mathrm{p} \pi^{-}$the value of $(\mathrm{d} \sigma / \mathrm{dt})_{\mathrm{t}=0}$ is well above that of the square of the imaginary part of the charge-exchange amplitude as calculated using the optical theorem, assuming charge independence, from the difference between the $\pi^{-} \mathrm{p}$ and $\pi^{+} \mathrm{p}$ 
total cross sections. ${ }^{4}$ This implies a non-zero difference between the real parts of the $\pi \mathrm{p}$ and $\pi^{+}$p elastic-scattering amplitudes.

In Table 4.I, Column 1 is the incident-pion lab morentum. Column 2 is the difference $\Delta \sigma$ between the $\pi^{-} p$ and the $\pi^{+} p$ total cross sections. ${ }^{4}$ Columin 3 shows the square of the imaginary part of the charg-exchange amplitude as calculated from $\Delta \sigma$. Column' 4 contains our values of $(\mathrm{d} \sigma / \mathrm{dt})_{\mathrm{t}=0}$ for the charge-exchange cross section, including the systematic as well as statistical errors. Column 5 is the square of the real part of the charge-exchange amplitude, given by the difference between the values in Columns 4 and 3 . Column 6 gives the absolute value of the difference between the real parts of the $\pi^{+} p$ and $\pi^{-} p$ elastic-scattering amplitudes at $t=0$, given by the square root of two times the value listed in Column 5 .

Table 4. I

The calculation of the absolute value of the difference between the real parts of the $\pi^{+} p$ and $\pi^{-} p$ elastic-scattering amplitudes at $t=0$, using our data and the total $\pi^{+} p$ and $\pi^{-} p$ cross sections from reference 4.

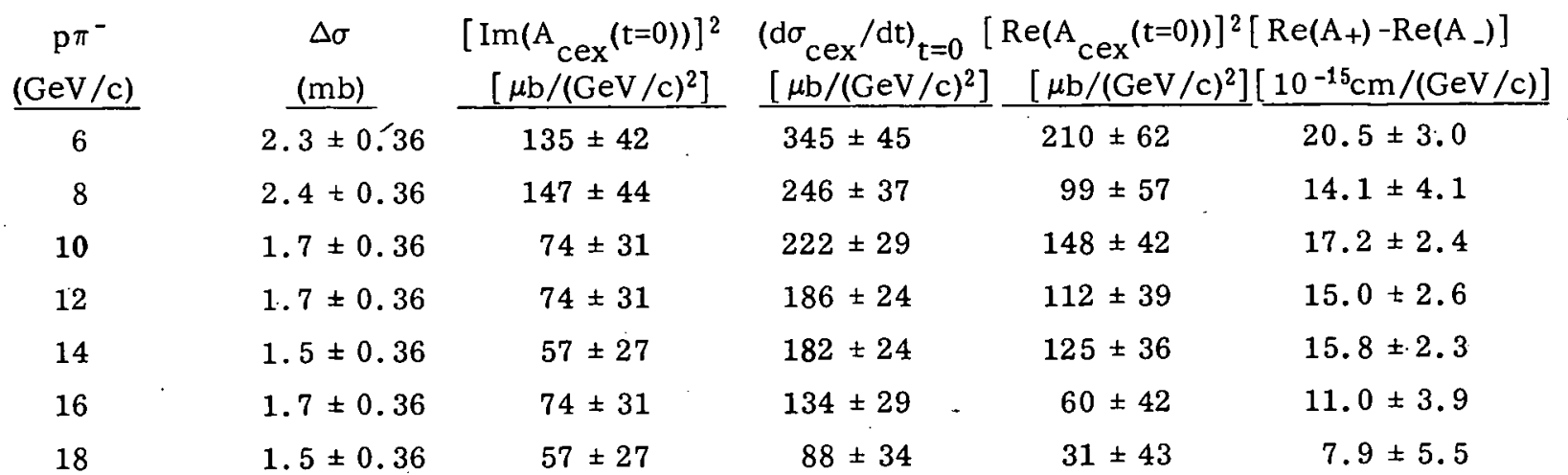

The differential cross section $(\mathrm{d} \sigma / \mathrm{dt})_{\mathrm{t}=0}$ decreases approximately as $1 / \mathrm{p}_{\pi}$ - and the real and imaginary parts have about the same magnitude (Fig. 4.5 (a) and Table 4. I). This is consistent with dispersion theory, which predicts equality of the real and imaginary parts when $(d \sigma / d t) t=0$ falls off as $1 / \mathrm{p}_{\pi^{-}}$.

In Figure 4.5 (b) are shown the values of the differential charge-exchange cross sections integrated between $t=0$ and $t=-0.4(\mathrm{GeV} / \mathrm{c})^{2}$. These integrated values include about 95 percent of the total charge-exchange cross sections if the differential cross sections, as shown in Fig. 4.4, continue to decrease exponentially for larger values of $-t$. 
High Energy Accelerator Physics Group

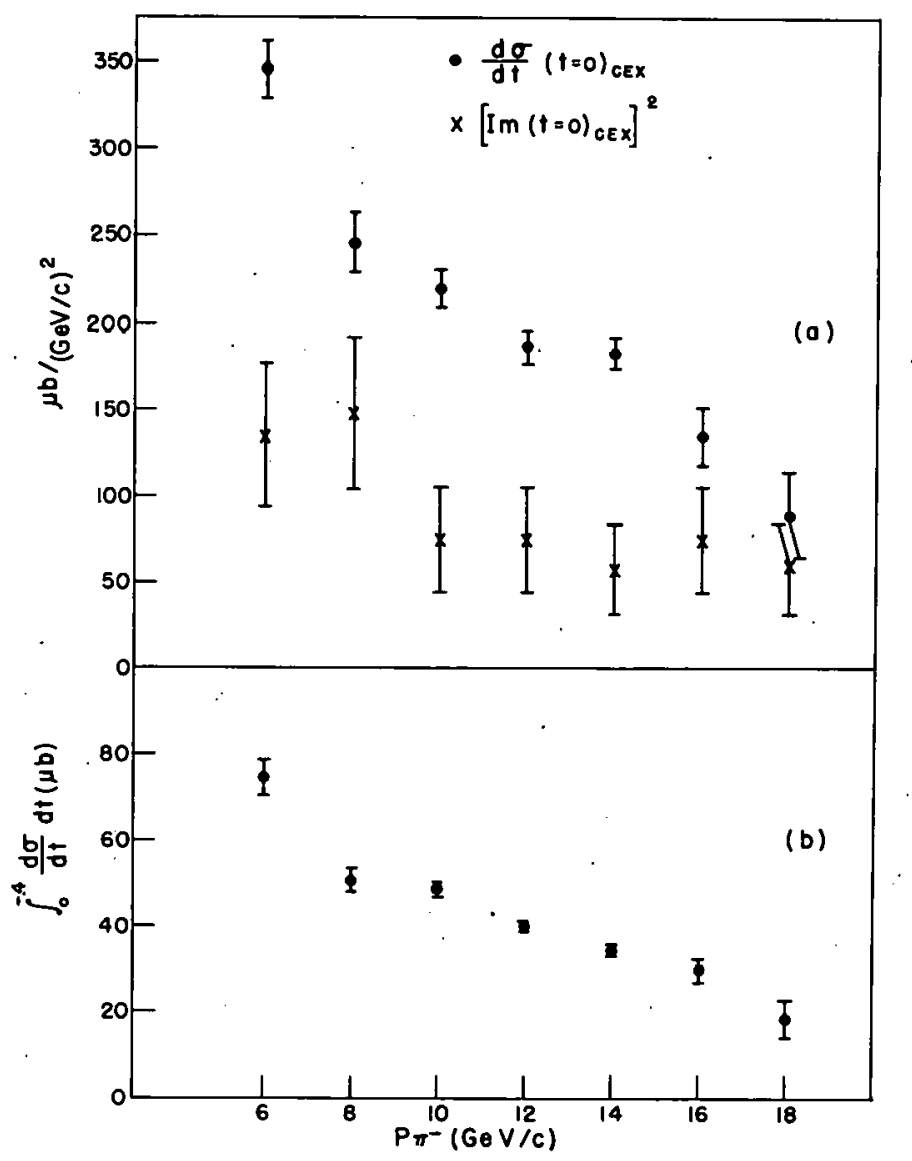

Figure 4.5

(a) The differential charge-exchange cross section at $t=0$ as a function of incident-pion lab momentum. As explained in the text, these values are obtained from the data in Fig. 4.4 by averaging the first three data points at each momentum. The errors shown are statistical only. For comparison, we have plotted the square of the imaginary part of the charge -exchange amplitude at $t=0, o b$ tained from data in reference 4.

(b) The values of the differential charge-exchange cross sections integrated between $t=0$ and $t=-0.4 \mathrm{GeV} / \mathrm{c}^{2}$. If the differential cross sections, as shown in Fig. 4.4, continue to decrease exponentially for larger values of $-t$, these integrated values include about 95 percent of the total charge-exchange cross sections.

Work continues on the angular distribution for elastic charge exchange in the region 2.4 $3.8 \mathrm{GeV} / \mathrm{c}^{5}{ }^{5}$ (M. Wahlig, L. Sodickson, I. Mannelli)

B. The $2 \pi^{0}$ Mass Spectrum and Determination of the Spin of the $\mathrm{f}^{0}$

The properties of the $\pi^{0}-\pi^{0}$ system have been studied using the interaction $\pi^{-}+p \rightarrow \pi^{0}+\pi^{0}+n$ at $10 \mathrm{GeV} / \mathrm{c}^{6,7}$

There is no unambiguous way to reconstruct the $2 \pi^{0}$ kinematics from the angles of the four gamma rays. However, with the following method and assumptions, a good approximation to the kinematics can be made on a restricted sample of the data. 
Assuming that each $\pi^{0}$ decays such that the opening angle between its two gammas is at a minimum (corresponding to a 90 -degree decay in the $\pi^{0}$ rest frame), the total energy of the $\pi^{0} \mathrm{~s}$ for each pairing of the gammas is calculated. The most likely correct pairing has its total energy nearest to the incident $\pi^{-}$energy $(10 \mathrm{GeV})$. This pairing is used in the subsequent analysis.

To determine the kinematics, one $\pi^{0}$ is assumed to decay with minimum opening angle and the other $\pi^{0}$ is assumed to have its direction approximated by the bisector of its gammas. Under this hypothesis, one kinematics solution can be calculated. With the roles of the two $\pi^{0} \mathrm{~s}$ reversed, a second kinematics solution is obtained. The two solutions are each used with a weight of $\frac{1}{2}$.

To validate this method and its assumptions, for both solutions the opening angle of the gammas from the $\pi^{0}$ not assumed to be at minimum was required to be within 0.9 times minimum to 1.2 times minimum (in the $\pi^{-p} \mathrm{c} . \mathrm{m}$.) if the event was to be retained. This restricted the sample of data events used to 19 percent of the total.

The $\pi^{0}-\pi^{0}$ mass spectrum is shown in Fig. 4.6 and shows two prominent enhancements over phase space. The first is the $\mathrm{f}^{0}$ meson, whose existence in the $2 \pi^{0}$ mass spectrum was the subject of an earlier paper. ${ }^{8}$ This peak is centered at $1275 \pm 25 \mathrm{MeV}$ and is consistent with a full width at half maximum of $100 \mathrm{MeV}$. B reit-Wigner ( $\mathrm{B}-\mathrm{W})$ fits to this $\mathrm{f}^{0}$ peak, generated by a Monte Carlo calculation using a width of $100 \mathrm{MeV}$, are shown drawn in on top of the phase space curves in Fig. 4.6.

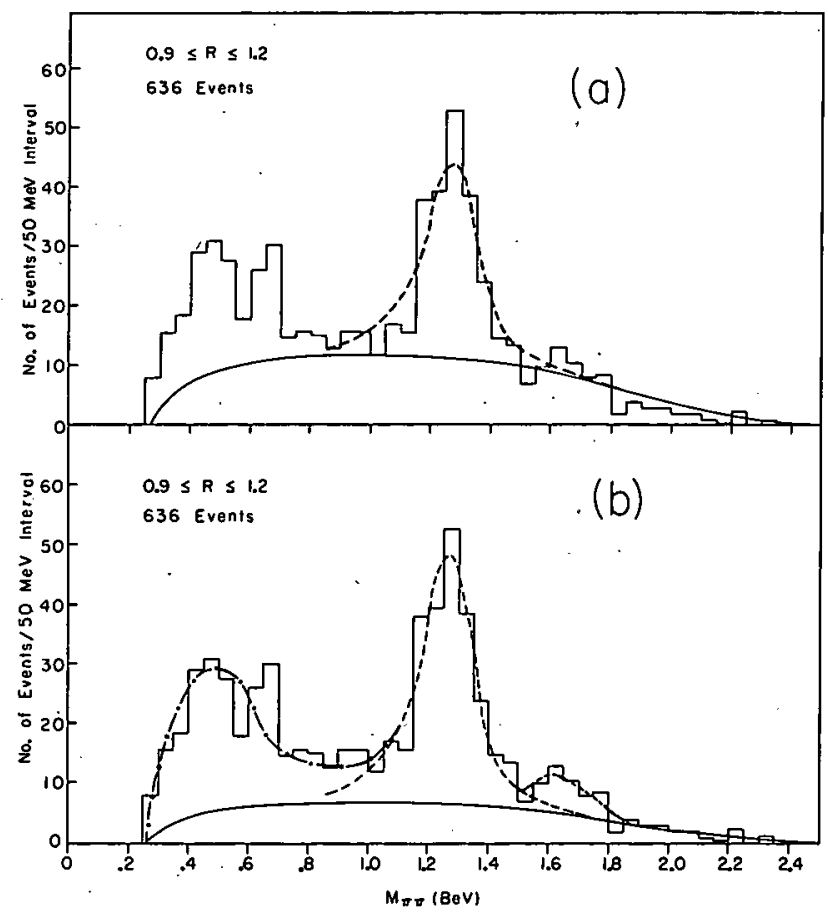

Figure 4.6

The $2 \pi^{0}$ mass spectrum. (a) Best fit using only phase space (solid line) plus $\mathrm{f}^{0}$ peak (dashed line). (b) Best fit using phase space (solid line), $\mathrm{f}^{0}$ peak (dashed line), low mass enhancement (dot-dash line), and $\mathrm{g}^{0}$ peak (dotted line). 
The second enhancement stretches across the low end of the mass spectrum, extending from threshold up to $\sim 800 \mathrm{MeV}$. We are still attempting to see if this enhancement can be due to contamination from multi-gamma $(>4)$ events, where only four gammas are detected, or $\eta \rightarrow \pi^{0}+\gamma+\gamma$.

The major uncertainty in obtaining a better fit to the mass spectrum is the detailed shape of the LME. Accordingly, as a rough approximation, and mainly for normalization purposcs, a fit to the mass spectrum was made using for the LME a B -W curve generated with its center at $550 \mathrm{MeV}$ and with a full width at half maximum of $300 \mathrm{MeV}$.

In view of the recent discovery of the $\mathrm{g}^{0}$ meson at $1670 \mathrm{MeV}$, which might decay into $2 \pi^{0} \mathrm{~s}$, a $\mathrm{g}^{0} \mathrm{~B}-\mathrm{W}$ curve, generated with its center at $1700 \mathrm{MeV}$ and a width of $100 \mathrm{MeV}$, was also included $\gamma$ in the fit to the mass spectrum. 9

The curve in Fig. 4.6(b) is the least-squares fit to the mass spectrum including these Monte-Carlo-generated $B-W$ curves for the LME, $f^{0}$, and $g^{0}$, as wel $l$ as phase space. This fit gives the following numbers of events: $174 \pm 21$ for the $\mathrm{f}^{0}$, and $135 \pm 11$ for the LME (taking the LME to be all events above phase space from threshold to $825 \mathrm{MeV}$ ).

Since it is impossible to choose, on the basis of these data, whether the fit in Fig. 4,6(a) or that in Fig. 4.6(b) is a better interpretation of the mass spectrum, this uncertainty must be included in the errors assigned to the cross sections. Accordingly, we give the following production cross sections at $10 \mathrm{GeV} / \mathrm{c}: 19 \pm 5 \mu \mathrm{b}$ for the $\mathrm{f}^{0}$ and $2 \pm 2 \mu \mathrm{b}$ for the $\mathrm{g}^{0}$. Since we observe only neutrals, all cross sections given are understood to be the product of the production cross section times the branching ratio for $2 \pi^{0}$ decay. Since one of our fits is consistent with no $\mathrm{g}^{0}$ events, this $\mathrm{g}^{0}$ cross section must be considered as an upper limit.

The $t$ dependence of the production cross section is approximately the same for all $\pi^{0}-\pi^{0}$ masses ( $t$ is the four-momentum transfer squared). Therefore a good approximation to the $\mathrm{f}^{0}$ differential production cross section is obtained by using the events in the 1150 to $1450 \mathrm{MeV}$ mass region and normalizing to the total $\mathrm{f}^{0}$ production cross section $(19 \mu \mathrm{b})$. The same is done for the LME region, using a mass cut between 400 and $700 \mathrm{MeV}$. These distributions are show $\mathrm{n}$ in Fig. 4.7. In both, $d \sigma / d t$ falls off initially as $e^{A t}$, where $A \approx 10$, which is similar to the $\pi^{-} p$ and $\mathrm{p}-\mathrm{p}$ behavior at this energy.

In Fig. 4.8 the $\pi-\pi$ scattering distributions are shown. The angle $\theta \pi \pi$ is defined as the angle between the incident $\pi^{-}$and one of the outgoing $\pi^{0} \mathrm{~s}$, in the di-pion c.m. system. Since the two final $\pi^{0} \mathrm{~s}$ are indistinguishable, these distributions are symmetric about 90 degrees.

When the di-pion system is in a state of $\operatorname{spin} \mathrm{J}$, the $\pi-\pi$ scattering should be described by the distribution $\left(\mathrm{P}_{\mathrm{J}}\left(\cos \theta_{\pi \pi}\right)\right)^{2}$, where $\mathrm{P}_{\mathrm{J}}$ is the $\mathrm{J}^{\text {th }}$ Legendre Polynomial. Figures 4.8(a) and 4.8(b) refer to the $\mathrm{f}^{0}$ mass region. The experimental histogram is compared with the Monte Carlo OP $\mathrm{E}$ predictions of a spin 0 , spin 2 , or spin 4 resonance, normalized to the same area. Here the effects of our small detection solid angle a re very noticeable. If we had full $4 \pi$ detection solid angle, the spin 0 prediction would be a straight horizontal line, and the spin 2 and spin 4 predictions would peak sharply at $\cos \theta_{\pi \pi}=1.0$. 
High Energy Accelerator Physics Group

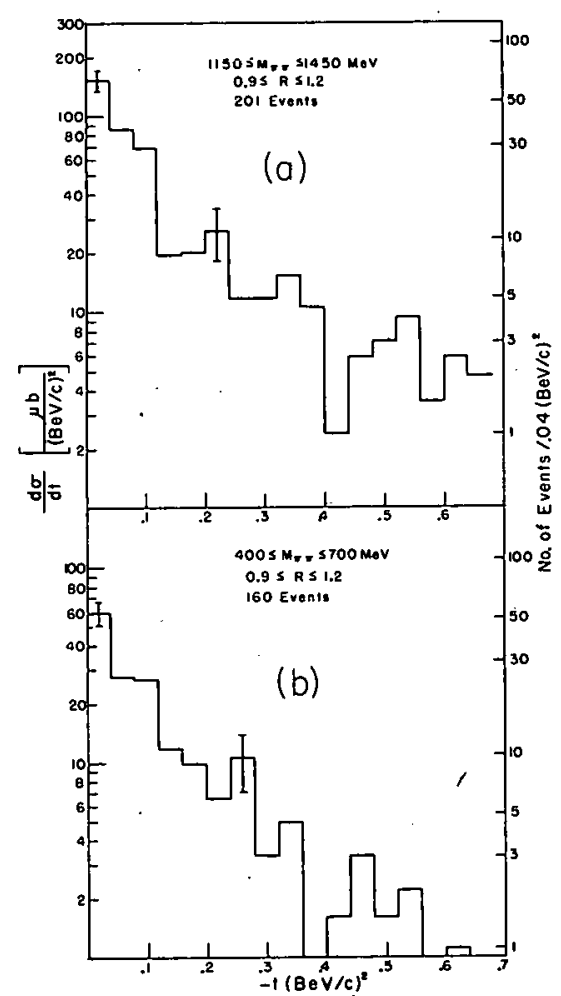

Figure 4.7

Differential production cross sections for (a) the $\mathrm{f}^{0}$ mass region and (b) the low mass region. These are normalized to total production cross sections of $19 \mu \mathrm{b}$ and $7 \mu \mathrm{b}$, respectively. The number of events per $t$ interval and a few statistical error bars are shown.

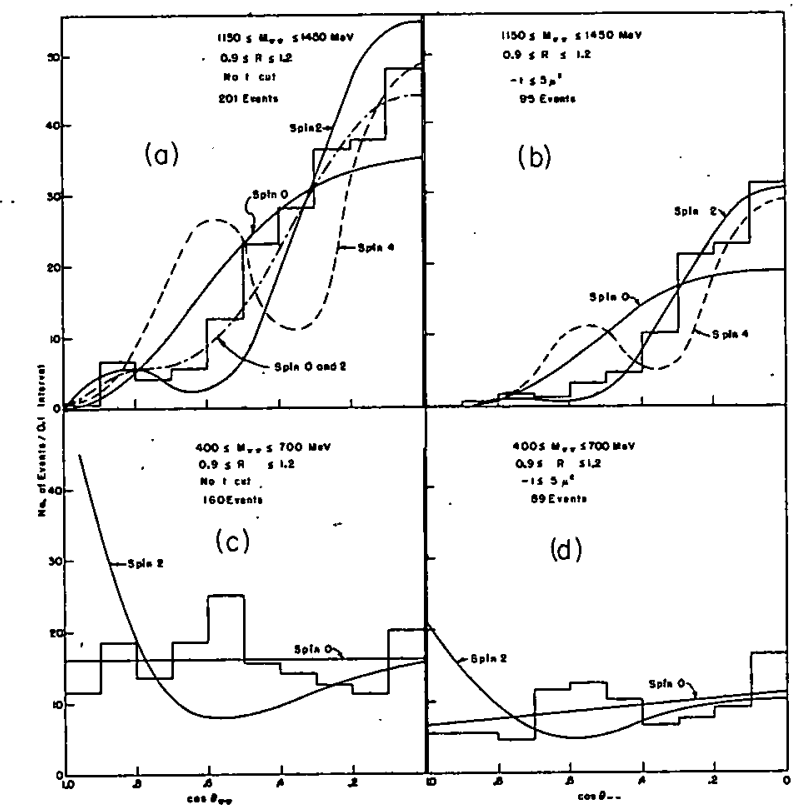

Figure 4.8

Distributions in $\cos \theta \pi \pi$. (a) and (b) refer to the $\mathrm{f}^{0}$ mass region without and with a $t$ cut. (c) and (d) refer to the low mass region without and with a t cut. The curves are predictions of various spin assignments for the $2 \pi^{0}$ system. 
Figure 4.8 (a) shows that, at the $\mathrm{f}^{0}$ mass with no cut in the four-momentum transfer, the $\cos \theta_{\pi \pi}$ distribution falls between the spin 0 and spin 2 curves and is fit very well by an incoherent mixture of approximately equal parts of spin 0 and spin 2 .

In Fig. 4.8 (b), we have made a cut in $-\mathrm{t}$ at $5 \mu^{2}$, where $\mu$ is the mass of the $\pi^{-}$, to limit the data to the region where OPE is most applicable. The figure shows that the data agree with pure spin 2, and are inconsistent with either spin $U$ or spin 4.

We conclude that the spin of the $\mathrm{f}^{0}$ is 2 and that in this mass region the background $\theta_{\pi \pi}$ distribution is predominantly isotropic.

In similar distributions for the $\mathrm{g}^{0}$ mass region, with very limited statistics, the data suggest spin 2 more than spin 0 or spin 4 . This is also what we would expect if these events are merely the high energy tail of the $\mathrm{f}^{0}$. Accordingly, these spin assignments were used in the calculation of the geometric detection efficiencies given above. (M. Wahlig, E. Shibata, D. Gordon, D. Frisch)

C. $\pi^{-}+\mathrm{p} \rightarrow 3 \gamma+\mathrm{n}$ at $10 \mathrm{GeV} / \mathrm{c}$

Work continues on the analysis and we are looking especially for $\omega^{0} \rightarrow \pi^{0}+\gamma$ and $\varphi \rightarrow \eta+\gamma$. (M. Wahlig, E. Shibata)

D. $\pi^{ \pm}+\mathrm{p} \rightarrow \pi^{0}+\pi^{ \pm}+\mathrm{p}$

The analysis of the 35,000 pictures taken at $964 \pm 14 \mathrm{MeV} / \mathrm{c}$ incident momentum has been completed and described in the thesis by $\mathrm{P}$. Mockett. ${ }^{10}$ The $\pi^{-} \pi^{0}$ mass spectrum was consistent with phase space (Figure 4.9), and no evidence for the $\pi^{-} \pi^{0}$ resonance reported around $575 \mathrm{MeV}$ was found. $11,12,13,14$ The $\pi^{-} \pi^{0}$ production cross section was determined to be $5 \pm 1 \mathrm{mb}$ at this energy. Analysis of the pictures at other $\pi^{-}$incident momenta -- about 30,000 pictures around 850 and $1200 \mathrm{MeV} / \mathrm{c}$ each -- and of 150,000 pictures with incident $\pi^{+}$momentum at $964 \pm$ $14 \mathrm{MeV} / \mathrm{c}$ is continuing. (P. Mockett)

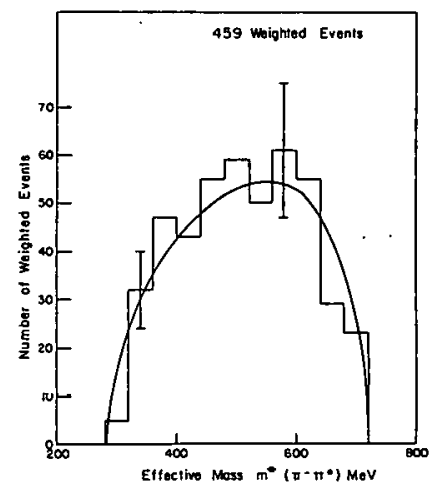

Figure 4.9

$\pi^{+} \pi^{0}$ invariant mass spectrum 


\section{E. $\pi^{-}+\mathrm{p} \rightarrow \mathrm{K}^{0}+\overline{\mathrm{K}}^{0}+\mathrm{n}$}

The Mascot system, consisting of very large solid angle spark chambers and scintillation counter hodoscopes in a uniform magnetic field (Argo magnet), was tested at BNL. It was operated for a short run of $10^{5}$ pictures to observe the reaction $\pi^{-}+\mathrm{P}$ goes to $\mathrm{V}$ particles plus neutrals. An illustrative picture is shown in Fig. 4.10 (a) and its reconstruction in real space in Fig. 4.10 (b).
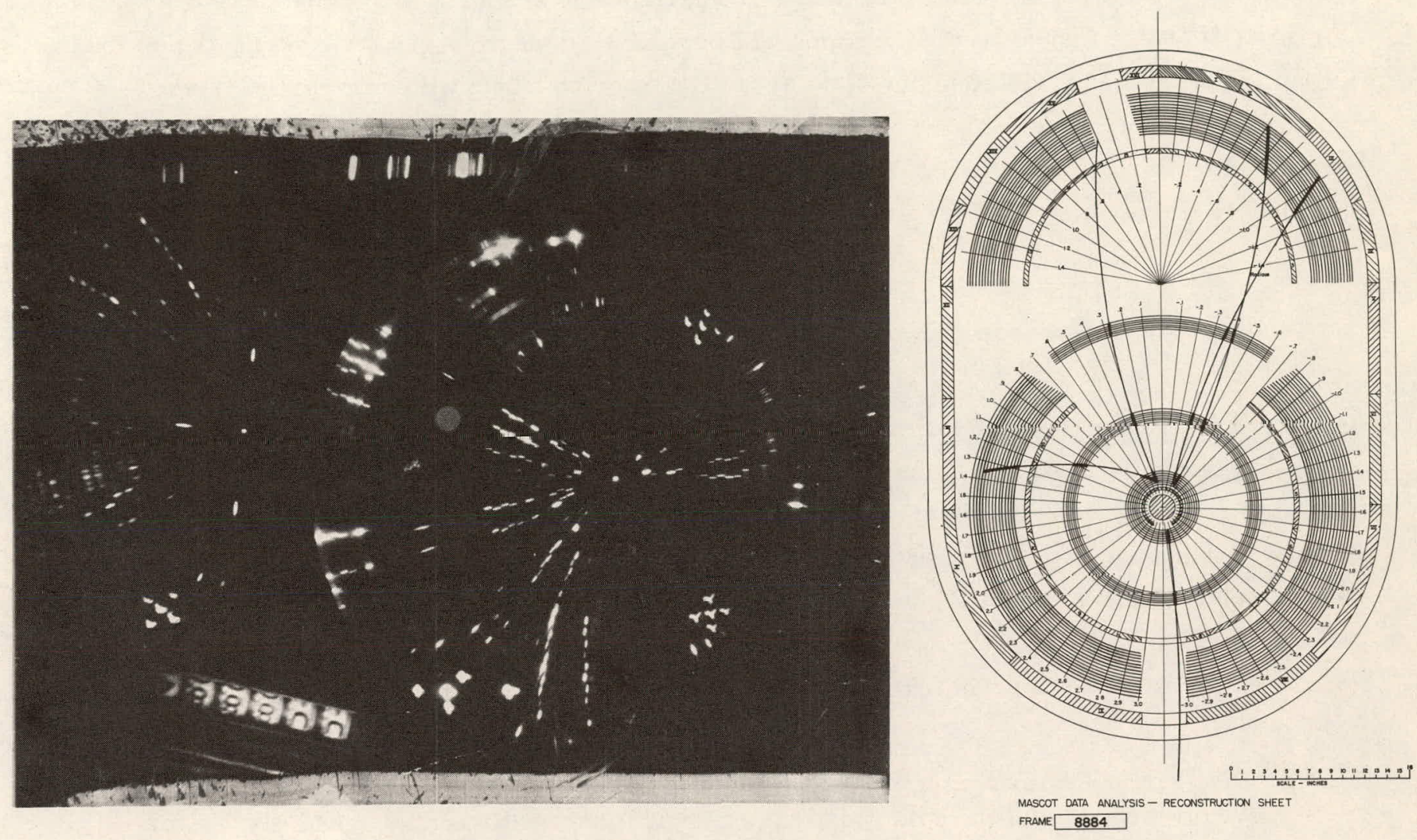

Figure 4.10

Illustration of the reaction $\pi^{-}+\mathrm{P}$ goes to $\mathrm{V}$-particles plus neutrals in (a) and its reconstruction in real space in (b).

Approximately 75 percent of these pictures has been scanned and measured. The programs for reconstruction of events from the film, for fitting and identification, and for efficiency determination are mostly complete.

A 300 -hour run with 8 to $12 \mathrm{MeV} / \mathrm{c} \mathrm{K}^{-}$has been approved and scheduled for November 1965 at $\mathrm{BNL}$. The reaction $\mathrm{K}^{-}+\mathrm{P} \rightarrow$ neutrals will be studied. (A. Buffington, C. Ward, S. Smith)

\section{F. CEA Internal Tagged Gamma Beam}

The detailed design work is almost complete for all major components of the internal tagged gamma beam. The rough design for the detection equipment to study the reaction $\gamma+C \rightarrow e^{+}+e^{-}$ 
(or $\mu^{+}+\mu^{-}$) $+\mathrm{C}$ is complete. The special donut section is under construction and the necessary electronics components are about half complete. (S. Gray, S. Smith)

G. $\Delta S=\Delta Q$ Rule, $C P$ and $C$ Violations in $K_{1}^{0}$ Decays

A 50 -hour test run at BNL to study the production characteristics of $\mathrm{K}^{0} \mathrm{~s}$ from the reaction $\mathrm{K}^{+}+\mathrm{W} \rightarrow \mathrm{K}^{0}+$ neutrons or short-ranged charged particles in a tungsten target has been approved for late fall 1965. Hopefully, this reaction will provide an intense source for investigation of the $\Delta \mathrm{S}=\Delta \mathrm{Q}$ rule, $\mathrm{CP}$ and $\mathrm{C}$ violations in $\mathrm{K}$ lecays. The tungsten target with wrap -around veto scintillation counter is now under construction and the spark chambers and scintillators for insertion in the large (Argo) magnet and the electronics are being designed. (O. Fackler, A. Hanson, H. Sartorio, D. Underwood)

\section{Electron and Photon Interaction Work}

\section{A. The Photoproduction of Wide Angle Muon Pairs}

We are now analyzing the data from a second set of measurements of the photoproduction of $\mu$-pairs from carbon, carried out in collaboration with physicists from Northeastern University. The second experiment yielded 400,000 analyzable events as compared to 10,000 from our first experiment. We thus expect to improve considerably the limits placed by our first experiment on a possible breakdown in the theory of quantum-electrodynamics as applied to the pair production process. (J. I. Friedman, G. Glass)

\section{B. Elastic Electron-Deuteron Scattering}

We have completed our data runs, having measured the differential cross section for elastic electron deuteron scattering in the range of four momentum transfer $3.75-6.2 \mathrm{f}^{-1}$. A preliminary analysis of the data at momentum transfers up to $4.75 \mathrm{f}^{-1}$ indicates good agreement of the measured form factor with predictions from models of the $n-p$ interaction having a repulsive core and about 7 percent $\mathrm{D}$ state. The analysis is being continued to the higher momentum transfers, and is being extended to test additional models, such as boundary value and finite repulsive core models. (J. de Pagter (CEA), J. Elias, J. I. Friedman, G. Hartman, H. W. Kendall, L. Van Speybroeck)

\section{The Two-Nucleon Correlation Function}

All of the primary data for this experiment have been analyzed with the use of our radiative correction computer programs. The experiment will soon be written up for publication. (Experiment in collaboration with Dr. D. Isabelle, Orsay) (H. W. Kendall) 


\section{Phase-Shift Analysis of Inelastic High Energy Scattering}

An initial version of this program has been tested and debugged. Square well and delta function nuclear charge densities were employed. A set of subroutines which will calculate the required potentials for arbitrary point specified densities has been written and debugged. The predictions of the calculation will soon be compared with experimental scattering results.

(J. I. Friedman, H. W. Kendall)

E. Search for the $e^{\prime}$

Low ${ }^{15}$ has suggested that a heavy electron $e^{\prime}$ might exist which could be formed in the reaction $\mathrm{e}+\mathrm{p} \rightarrow \mathrm{e}^{\prime}+\mathrm{p}$.

A search was carried out for this reaction by observing the spectrum of recoil protons. A preliminary analysis of this data indicates that the cross section for this reaction is less than about 5 percent of the elastic scattering cross section for an $e^{\prime}$ mass up to about $1100 \mathrm{MeV}$. We are now in the midst of a final analysis of the data. (A. Broadfoot, J. Elias, J. Friedman, G. Hartman, H. Kendall, P. Kirk, M. Sogard, L. Van Speybroeck)

\section{F. External Beam Facilities}

The quadrupole spectrometer with which we carried out the elastic electron deuteron scattering program is to be moved from its present location in the accelerator tunnel to an external electron beam. We are making preparations for the following experiments to be done with the new facility.

1. Elastic electron $-\alpha$ particle scattering up to four momentum transfers of $6 \mathrm{f}^{-1}$.

2. Coherent photoproduction of $\pi^{0}$ mesons from the deuteron at photon energies of $1-3 \mathrm{BeV}$.

3. Measurements of the final state interaction in the electro-disintegration of the deuteron.

4. Measurements of the photo-production of the $\eta^{0}$ and $\rho^{0}$ mesons at large momentum transfers.

(A. Broadfoot, J. Elias, J. I. Friedman, G. Hartman, H. W. Kendall, P. Kirk, M. Sogard,

L. Van Speybroeck)

\section{G. SLAC Collaboration}

, We are now making preparations for a collaboration with the group at SLAC that will be engaged in the study of electron -proton scattering. Our group will be responsible for the design and construction of the particle detector hodoscopes and the associated electronics to be employed in the 8 and $20 \mathrm{BeV}$ spectrometers which are being designed for the electron scattering program. Our efforts are now directed to the following objectives.

1. The design and construction of the hodoscopes to be placed in the focal planes of the spectrometers.

2. The design and construction of a detector that will be able to distinguish electrons and $\pi^{-}$ mesons of the same momenta in the range of $20 \mathrm{BeV} / \mathrm{c}$ to about $1 \mathrm{BeV} / \mathrm{c}$ : 
3. The design and construction of the electronic logic associated with the detectors and the slow electronic logic used to read the information into an on -line computer.

4. The construction of a radiative correction program to be used as an aid in designing experiments.

(M. Breidenhach, J. I. Friedman, H. W. Kendall, L. Van Speybroeck)

\section{REFERENCES}

1. I. Mannelli, A. Bigi, R. Carrara, M. Wahlig, and L. Sodickson, Phys. Rev. Letters 14 , 408 (1965).

2. MIT Laboratory for Nuclear Science Progress Report, Nov. 1, 1964, MIT-2098-142.

3. S. J. Lindenbaum, P roceedings of the International Conference on Nucleon Structure at Stanford University, 1963, ed. R. Hofstadter and L. I. Schiff (Stanford University Press, Stanford, California, 1964), p. 105.

4. W. Galbraith, E. W. Jenkins, T. F. Kycia, B. A. Leontic, R. H. Phillips, A. L. Read, and R. Rubinstein (to be published).

5. M. A. Wahlig, I. Mannelli, L. Sodickson, O. Fackler, C. Ward, T. Kan, and E. Shibata, Phys. Rev. Letters 13,103 (1964).

6. M. A. Wahlig, L. Sodickson, I. Mannelli, D. Frisch, and O. Fackler, Phys. Rev. Letters 12 , (April 1964).

7. LNS Progress Report, op. cit.

8. M. A. Wahlig (to be published).

9. CERN, Ecole Polytechnique, and Orsay, Milan, Saclay Collaboration, Phys. Letters 17, 354 (1965).

10. P. M. Mockett, High $-Z$ Cylindrical Spark Chamber Study of the Reaction $\pi^{-}+\mathrm{P} \rightarrow \pi^{-}+\pi^{0}+\mathrm{P}$ at $964 \mathrm{MeV} / \mathrm{c}, \mathrm{Ph} . \mathrm{D}$. Thesis, Physics Department, MIT, May 14, 1965.

11. E. Pickup, F. Ayer, and E. O. Salant, P hys. Rev. Letters 5, 161 (1960).

12. R. Berloutaud, J. Heughebaert, A. Leveque, and J. Meyer, $\overline{\mathrm{P}}$ hys. Rev. Letters $\underline{8}, 32$ (1962).

13. C. Peck, L. Jones, and M. Perl, Phys. Rev. 126, 1836 (1962).

14. S. Zorn, Phys. Rev. Letters 8,262 (1962).

15. F. E. Low, Phys. Rev. Letters, 14, 238 (1965).

\section{PUB LICATIONS}

H. Brechna, D. A. Hill, B. M. Bailey, "A 150 kOe liquid Nitrogen Cooled Pulsed Flux-Concentrator Magnet", Rev. Sci. Instr. 36, No. 11, 1529-1535 (Nov. 1965).

D. O. Caldwell, J. P. Dowd, K. Heinloth, M. D. Rousseau," "Tagging System"for High Energy Photons", Rev. Sci. Instr. 36, No. 3, 283-288 (March 1965).

B. T. Feld, L. D. Roper, R. M. Wright, "Energy Dependent Pion-Nucleon Phase Shift Analysis", Phys. Rev. 138, No. 1B (April.1965).

I. Marelli, A. Bigi, R. Carrara (P isa, Italy), M. Wahlig, L. Sodickson (MIT), $" \pi^{-}+p \rightarrow \pi^{0}+n$ Charge Exchange Scattering at High Energy", Phys. Rev. Letters 14, No. 11, 408 (15 March 1965).

\section{CONFERENCES AND MEETINGS}

\section{Abstracts}

R. Alvarez, Z. Bar-Yam, D. Garelick, S. Homma, W. Kern, R. Lewis, W. Lobar, L. S. Osborne, J. Uglum, "P hòtoproduction of Neutral P ions at GeV Energies", DESY Conference, Hamburg, Germany (June 1965). 
M. Deutsch, J. Cleetus, E. Loh; P. Patel, "Elastic Photon-P roton Scattering in the Range 1.2 to $2.0 \mathrm{GeV}$ ", Am. Phys. Soc. Meeting, Washington, D. C. (April 1965).

D. Gordon, E. Shibata, D. Frisch, L. Sodickson, and M. Wahlig, "Study of $\pi^{-}+p \rightarrow 2 \pi^{0}+n$ and $\pi^{0}+\gamma+n$ at $10.0 \mathrm{~B} \mathrm{eV} / \mathrm{c}^{\prime \prime}$, Am. Phys. Soc. Meeting, Washington, D. C. (April 1965)

R. Lewis, D. Luckey, "A Total Absorption Gamma Ray Hodoscope", DESY Conference, Hamburg (June 1965).

J. R. Uglum, R. Alvarez, A. Bar-Yam, D. Garelick, S. Homma, W. Kern, R. Lewis, D. Luckey, L. Osborne (MIT); R. Fessel (CEA), "P hotoproduction of Charged and Neutral $\pi$ Mesons", Am. Phys. Soc. Meeting, Washington, D. C. (April 1965).

Addresses and Invited P apers

R. Alvarez, V. Elings, Z. Bar-Yam, D. Garelick, S. Homma, W. Kern, R. Lewis, D. Luckey, L. Osborne, J. Uglum, "A P hotoproduction Experiment using a Small Computer", presented at Purdue Conference on Instrumentation for High Energy Physics (May 1965).

J. DePagter (CEA), J. Friedman, H. Kendall, L. VanSpeybroeck, J. Elias, G. Hartman, "Elastic Electron Deuteron Scattering at High Momentum", Conference P roceedings, International Conference on Electron and P hoton Interactions, Hamburg, Germany (June 1965).

M. Deutsch, "Guided Flying Spot Systems for Spark Chambers", invited talk given at the P.urdue Conference on Instrumentation for High Energy Physics (May 1965), IEEE Trans actions on Nuclear Science, Vol. NS-12, No. 4 (August 1965).

L. S. Osborne, "Photoproduction of Mesons in the GeV Range", P roc. of the Conference on International Symposium on Electron and Photon Interactions at High Energies, DESY, Hamburg, Germany (June 1965); Springer Tracts in Modern Physics, Vol. 39 (Springer-Verlag, B erlin: 1965).

\section{THESES}

W. R Alves, Jr., "Differential Cross Section of $\pi^{+}$Photoproduction at $2.7 \mathrm{~B} \mathrm{eV}$ Incident Photon Energy" (with J. J. Nagy, Jr.), B.S. (June 1965).

S. Apsell, "Cross Section of the Photoproduced $1238 \mathrm{MeV} \mathrm{N}$ * Particle", B.S. (June 1965).

D. M. Bellenger, "Wire Spark Chamber using a Coincident Current Memory", B.S. (June 1965).

M. Breidenbach, "P ion-Electron Separation in an Ultra-Relativistic Momentum Selected Beam" B.S. (May 1965).

D. S. Cannell, "Coherent Photoproduction of $\pi^{0}$ Mesons from Deuterium", B.S. (May 1965).

S. B. Deutsch, "A Study of Bremsstrahlung Subtraction", B.S. (May 1965).

V. B. Elings, "P hotoproduction of Single $\pi^{+}$and $\mathrm{K}^{+}$Mesons from Hydrogen at Incident Energies near 4.0 BeV", Ph.D. (December 1965).

D. Ernst, "Resolution in a $2 \mathrm{~cm}$ Gap Spark Chamber" (with S. Gray), B.S. (June 1965).

R. K. Fisher, "The Construction and Operat ion of Thin-Foil Narrow-Gap Spark Chambers", B.S. (June 1965).

B. Goodman, "Magnetic Spectrometer Calculations for Large Angle $\pi^{+}$Photoproduction", B.S. (June 1965).

S. W. Gray, "Resolution in a $2 \mathrm{~cm}$ Gap Spark Chamber", B.S. (June 1965) (with D. Ernst).

E. S. Groves, "A Test of a Gas Differential Cerenkov Counter", B.S. (June 1965).

G. R. Johnson, "P ion Photoproduction", B.S. (May 1965).

R. J. Long, "Construction and Testing of a Broad Response Cerenkov Counter", B.S. (June 1965).

P. M. Mockett, "High Z Cylindrical Spark Chamber Study of the Reaction $\pi^{-}+p \rightarrow \pi^{-}+\pi^{0}+p$ at $964 \mathrm{MeV} / \mathrm{c}^{\prime \prime}, \mathrm{Ph} . \mathrm{D}$. (May 1965).

J. J. Nagy, Jr., "Differential Cross Section of $\pi^{+} \mathrm{P}$ hotoproduction at $2.7 \mathrm{BeV}$ Incident $\mathrm{P}$ hoton Energy" (With W.R. Alves, Jr.), B.S. (June 1965).

S. A. Schutz, "Construction of a Photographic Two Dimensional Pulse Height Analyzer", B.S. (June 1965).

A. R. Stottlemyer, "An Analysis of the Magnetic Field in a Spark Chamber from Straight Through Particle Tracks", B.S. (May 1965). 
- High Energy Accelerator Physics Group

M. W. Strovink, "The CP -Violating Decay K $\rightarrow 3 \pi$ ", B.S. (May 1965).

M. R. Teague, "Elastic Electron-Deuteron Scattering", B.S. (May 1965).

J. R. Uglum, Jr., "P hotoproduction of $\pi^{0}$ Mesons from 2.1 to $4.8 \mathrm{~B} \mathrm{eV}$ and $\pi^{+}$Mesons from 3.2 to $4.5 \mathrm{~B} \mathrm{eV}^{\prime \prime}$, Ph.D. (May 1965).

L. P. Van Speybroeck, "Elastic Electron Deuteron Scattering at High Momentum Transfer", Ph.D. (June 1965).

R. Wilson, "Determination of the Optimum Base for the 6910-A Photomultiplier Tube", B.S. (May 1965). 


\section{$\cdots$ \\ O.N.R. GENERATOR GROUP \\ O.N. \\ I. Introduction}

During the period covered by this report, a number of exposures have been made with $\mathrm{He}^{3++}$, deuteron, and proton beams. The data for most of these exposures are being analyzed here, but some have been sent to other universities; for example, the University of Bergen in Norway, University of Mexico, Yale University, Providence College, and the University of $\mathrm{Pittsburgh}$. The work with the $\mathrm{He}^{3}$ beam has proved to be extremely interesting. However, .... . with the old type ion source, very long exposure times have been required because the single charged $\mathrm{He}^{3}$ ions have outnumbered the doubly charged by more than 100 to 1 . Without terminal analysis, it is therefore necessary to run a quite intense $\mathrm{He}^{3+}$ beam in the tube in order to obtain a few nanoamps of $\mathrm{He}^{++}$. This causes extensive wear on the tube, and this is the main reason why further $\mathrm{He}^{3}$ work has been postponed until a new ion source has been installed. A new ion source with terminal analysis is now being tested.

In anticipation of a future acquisition of a Tandem accelerator, some design studies have been initiated on improved magnetic instruments for such a facility. These instruments include a 185-degree absolute-energy beam analyzer. ${ }^{1}$ The effect of coil geometry on the field in such a magnet has been worked out with a view toward minimizing second-order aberrations. The second instrument that is being studied is a high-resolution, multiple-gap magnetic spectrograph. An instrument with a resolving power of 1 part in 10,000 is being aimed for. A single-gap prototype for such an instrument is now being designed and will be erected outside the multiple-gap spectrograph vacuum tank and operated in conjunction with this latter equipment (see Section XIV).

The electronic components of the automatic nuclear-track scanner (Cyclops) have been completely revamped, improving the reliability of the circuitry and increasing the speed (band width) to match the new disk scanner. Operation with simulated track signals recorded on an unused channel of the drum memory indicates that the logic and memory perform in a satisfactory manner. The mechanical stage operates well when coupled to the revamped system. The major components of the optical link and illuminators have been obtained and assembled. Progress is now under way on aligning and testing the optical system; when this is completed, scanning tests will be initiated.

\section{Studies of Energy Levels in the $\mathrm{f}_{7} / 2$ Shell}

\section{A. The $\mathrm{Ca}^{42}(\mathrm{~d}, \mathrm{p}) \mathrm{Ca}^{43}$ Reaction}

The $\mathrm{Ca}^{42}(\mathrm{~d}, \mathrm{p}) \mathrm{Ca}^{43}$ reaction has been studied at 7.00 - and $7.20-\mathrm{MeV}$. bombarding energies. Eighty transitions were observed up to an excitation energy of $5.0 \mathrm{MeV}$. The angular distributions

1. H. A. Enge, Nucl. Instr. and Methods 28, 119 (1964). 
showing stripping character were analyzed in the distorted-wave approximation to yield $\ell_{n}$ values and spectroscopic strengths. A sum-rule analysis indicated that all of the available $1 f_{7} / 2,1 d_{3} / 2$, and $2 p$ strengths were observed. The beginnings of the $1 g_{9} / 2,1 f_{5} / 2$, and $2 d_{5} / 2$ transitions were recorded. The data suggest that neither the neutron nor the proton $1 \mathrm{~d}_{3} / 2$ shell is filled in the $\mathrm{Ca}^{42}$ ground state. The stripping data are presented in Fig. 5.1 in the form of a strength-function diagram. The results of this work have been submitted for publication to the Physical Review.

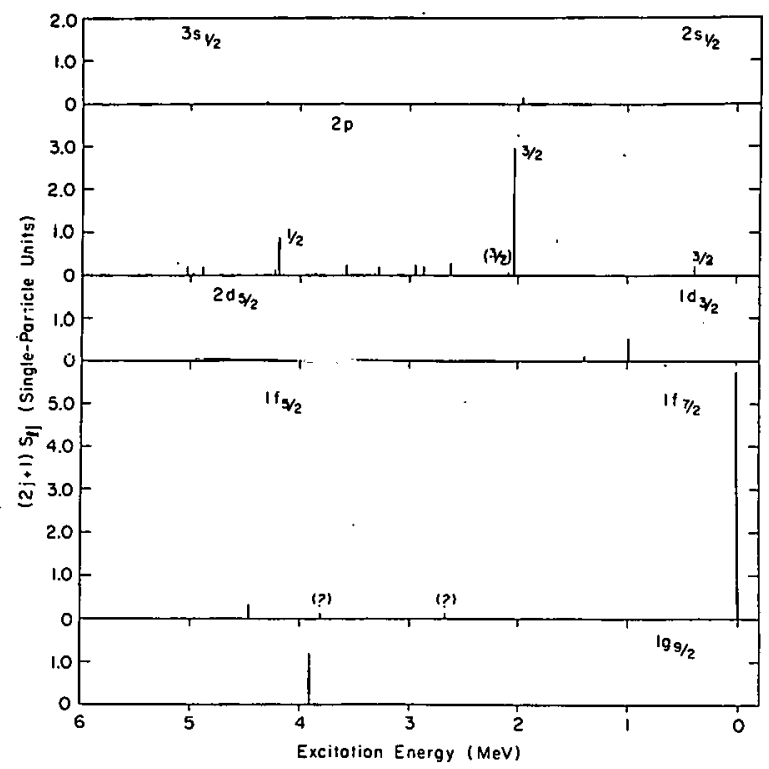

Figure 5.1 Strength function for $\mathrm{Ca}^{43}$. The values of $(2 \mathrm{~J}+1) \mathrm{S}_{\ell j}$
are plotted vs excitation energy.

B. Inelastic Deuteron Scattering

Eleven nuclei in the $f_{7} / 2$ shell have been investigated by the inelastic scattering of deuteruns. The favorable background conditions of the spectrograph have allowed us to study the weaker transitions to excited $0^{+}$states, to second $2^{+}$states, and to $4^{+}$states, in addition to the strong transitions to first $2^{+}$states and $3^{-}$states in the even nuclei. Together with the (d, d') work ${ }^{1}$ the present program covers all the $\mathrm{If}_{7} / 2$ shell nuclei available as targets, with the exception of $\mathrm{Ca}^{48}$. Energy levels up to $4 \mathrm{-MeV}$ excitation were studied.

In Fig. 5.2, the angular distributions from the transitions to the first excited states of $\mathrm{Ca}^{42}, \mathrm{Ca}^{44}, \mathrm{Ti}^{46}, \mathrm{Cr}^{50}, \mathrm{Fe}^{54}$, and the $912-\mathrm{keV}$ level of $\mathrm{V}^{50}$ are shown. In the first four cases, these states have measured $2^{+}$character. The shapes and cross-section magnitudes of the angular distributions for these transitions establish the direct character of the reaction mechanism at this energy. The results for $\mathrm{Fe}^{54}$ support the expected $2^{+}$character of this state. The $\mathrm{V}^{50}$ data suggest that a rather pure quadrupole transition is involved, thus limiting the possible spin values

1. T. A. Belote, J. H. Bjerregaard, Ole Hansen, and G. Satchler, Phys. Rev. 138, B1067 (1965). 
for the $912-\mathrm{keV}$ state to $4,5,6,7$, or 8 and positive parity, since $\mathrm{V}^{50}(0)$ has $\mathrm{J}^{\pi}=6^{+}$. A preliminary report of this work was presented at the Conference on Bases for Nuclear Spin-Parity Assignments, Gatlinburg, Tennessee, November $\overline{11-13,1965}$.

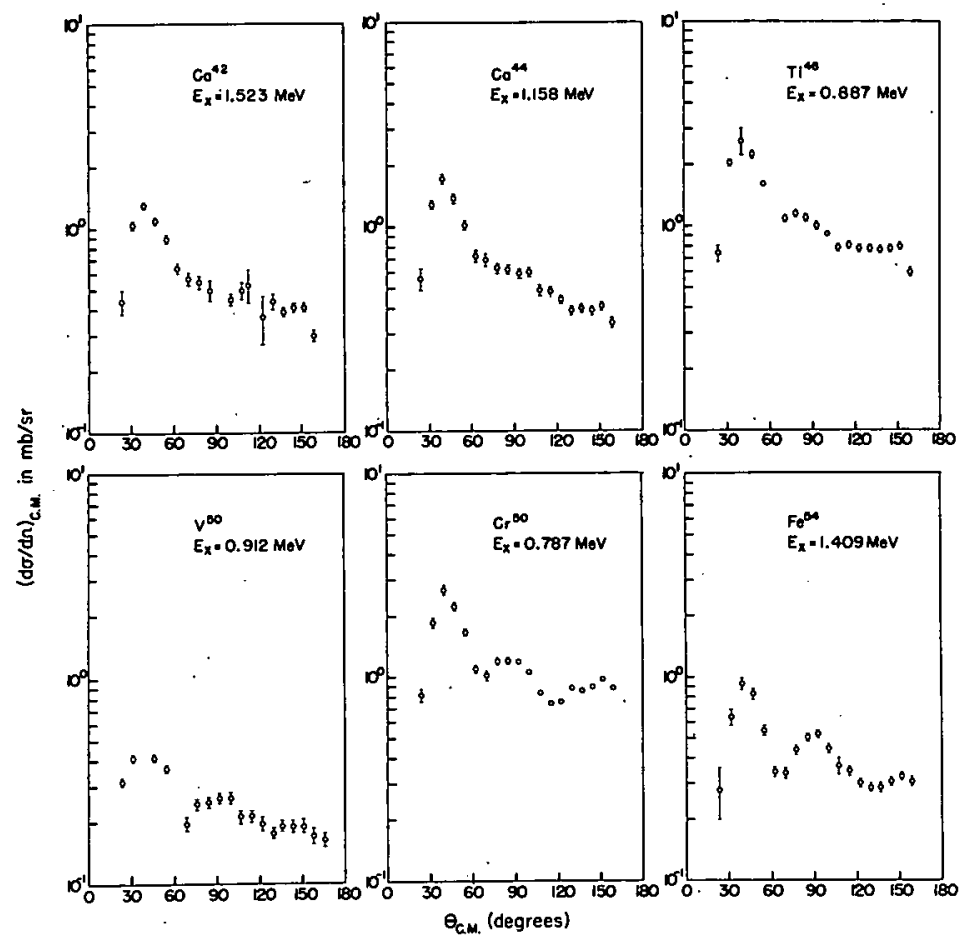

Figure 5.2

Angular distribution for $\mathrm{L}=2$ transitions. The angular distribution data for the $\mathrm{L}=2$ transitions are shown to the first excited state of $\mathrm{Ca}^{42}, \mathrm{Ca}^{44}, \mathrm{Ti}^{46}, \mathrm{Cr}^{50}$, and $\mathrm{Fe}^{54}$ and to the $912-\mathrm{keV}$ level of $\mathrm{V}^{50}$.

C. The $\mathrm{Ti}^{48}\left(\mathrm{p}, \mathrm{p}^{\prime}\right) \mathrm{Ti}^{48}$ Reaction

The results on this reaction have been published in $\mathrm{P}$ hysics Letters ${ }^{1}$. Of special interest is the new level in $\mathrm{Ti}^{48}$ at $3.004-\mathrm{MeV}$ excitation that has $0^{+}$character.

D. The $\mathrm{Ca}^{42}(\mathrm{~d}, \mathrm{p}) \mathrm{Ca}^{43}, \mathrm{Ca}^{44}(\mathrm{~d}, \mathrm{p}) \mathrm{Ca}^{45}$, and $\mathrm{Ti}^{46}(\mathrm{~d}, \mathrm{p}) \mathrm{Ti}^{47}$ Reactions to Low -lying $5 / 2^{-}$Levels

The $(d, p)$ reactions leading to the low-lying $5 / 2^{-}$levels in $\mathrm{Ca}^{43}, \mathrm{Ca}^{45}$, and $\mathrm{Ti}^{47}$ have been studied. Since these levels are mainly of an $\left(f_{7} / 2\right)^{ \pm 3}$ configuration, they cannot be excited by simply the transfer of an $f_{7} / 2$ neutron. Evidence has been obtained that suggests that these transitions are direct in character and that the final-nucleus excitation mode is a possible mechanism for these reactions. The data for these transitions are shown in Fig. 5.3. The results of this work have been published in Nuclear Physics. ${ }^{2}$

1. T.A. Belote, W. E. Dorenbusch, Ole Hansen, and A.Sperduto, Phys. Lett. 14, 323 (1965)

2. T. A. Belote, W. E. Dorenbusch, Ole Hansen, and J. Rapaport, Nucl. Phys. $\overline{73}, 321$ (1965). 


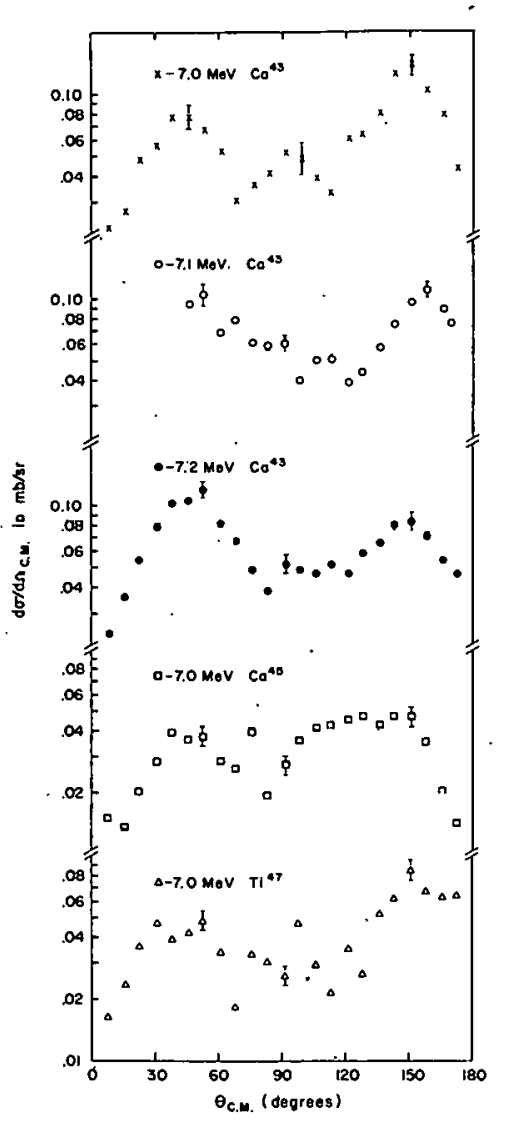

Figure 5.3

Angular distributions from $(d, p)$ transilions to the $5 / 2^{-}$states of $\mathrm{Ca}^{43}, \mathrm{Ca}^{45}$, and $\mathrm{Ti}^{87}$.

E. The $V^{50}(d, p) V^{51}$ Reaction

An exposure of $2500 \mu \mathrm{C}$ of $7.5-\mathrm{MeV}$ incident deuterons has been made. The analysis of this reaction is being done in conjunction with the group at the National University of Mexico. Angular distributions for about 120 levels have been extracted and are now being analyzed to obtain spectroscopic information.

F. The $\mathrm{V}^{50}(\mathrm{~d}, \alpha) \mathrm{Ti}^{48}$ Reaction

The $\mathrm{V}^{50}(\mathrm{~d}, \alpha) \mathrm{Ti}^{48}$ reaction has been examined at 7.50-MeV bombarding energy. Energy levels, several of which are new, up to $5.7 \mathrm{MeV}$ of excitation were identified. It is suggested that those $\mathrm{Ti}^{48}$ levels that are strongly excited in the $(\mathrm{d}, \alpha)$ reaction have spin between 6 and 10 . The results of this work have been submitted for publication to Nuclear Physics.

G. The $\mathrm{Ca}^{44}(\mathrm{~d}, \mathrm{p}) \mathrm{Ca}^{45}$ Reaction

An exposure of $5000 \mu \mathrm{C}$ at $7.0-\mathrm{MeV}$ bombarding energy has been made. The analysis is being carried out in conjunction with Dr. J. Rapaport of the University of Chile (presently at MIT). 
H. The $\mathrm{Ca}^{46}(\mathrm{~d}, \mathrm{p}) \mathrm{Ca}^{47}$ Reaction

Twenty-seven excited levels have been identified in $\mathrm{Ca}^{47}$ below an excitation energy of $6.1 \mathrm{MeV}$ from the $\mathrm{Ca}^{46}(\mathrm{~d}, \mathrm{p}) \mathrm{Ca}^{47}$ reaction. An $\ell_{\mathrm{n}}=2$ transition to the $2.580-\mathrm{MeV}$ level has been analyzed to give information on the ground-state configuration of $\mathrm{Ca}^{46}$. It is suggested that this state has a configuration

$$
\begin{aligned}
& \left|\mathrm{Ca}^{47}(2) 3 / 2+\begin{array}{l}
\mathrm{T}=7 / 2 \\
\mathrm{~T}_{\mathrm{z}}=7 / 2
\end{array}\right\rangle=\sqrt{\frac{8}{9}} \cdot\left\{\begin{array}{ll}
1 \mathrm{~d}_{3}^{-1} / 2 & \mathrm{~T}=1 / 2 \\
\mathrm{~T}_{\mathrm{z}}=-1 / 2
\end{array}\right\}\left\{\begin{array}{ll}
1 \mathrm{f}_{7 / 2}^{8} & \begin{array}{l}
\mathrm{T}=4 \\
\mathrm{~T}_{\mathrm{z}}=4
\end{array}
\end{array}\right\} \\
& -\sqrt{\frac{1}{9}}\left\{\begin{array}{ll}
1 \mathrm{~d}_{3 / 2}^{-1} & \mathrm{~T}=1 / 2 \\
\mathrm{~T}_{\mathrm{z}}=1 / 2
\end{array}\right\}\left\{\begin{array}{ll}
1 \mathrm{f}_{7 / 2}^{8} & \mathrm{~T}=4 \\
\mathrm{~T}_{\mathrm{z}}=3
\end{array}\right\} .
\end{aligned}
$$

This assumption leads to an admixture of about $0.2\left\{1 \mathrm{~d}_{3}^{-2}{ }^{2} \mathrm{~T}=1\right\} \times\left\{1 \mathrm{f}^{8} / 2^{. T=4}\right\}$ in the $\mathrm{Ca}^{46}$ ground state wave function. The results of this reaction have been published in the Physical Review. ${ }^{1}$.

\section{The Enriched Calcium Target Experiment}

In order to obtain more nearly accurate transition strengths for the even-calcium $(d, p)$, odd-calcium ground-state transitions, a special target containing approximately equal amounts of $\mathrm{Ca}^{40}, \mathrm{Ca}^{42}, \mathrm{Ca}^{44}, \mathrm{Ca}^{46}$, and $\mathrm{Ca}^{48}$ has been prepared. Exposures. at 7.0 and $7.2 \mathrm{MeV}$ have been made, and the data are presently being scanned. The targets were specially prepared by A. P. Luongo and were about $12 \mu \mathrm{g} / \mathrm{cm}^{2}$ in thickness.

\section{J. Targets Produced in an Isotope Separator}

An association with G. Sidenius of the Isotope Separator at Princeton University and with Professor R. Nauman of the Princeton Chemistry Department has been initiated. Better than 99 percent enriched targets of $V^{50}$ have been produced. The $(d, p)$ and $(d, \alpha)$ reactions on these targets are described above. The $\mathrm{V}^{50}\left(\mathrm{~d}, \mathrm{~d}^{\prime}\right) \mathrm{V}^{50}$ scattering process is now being studied. (T. A. Belote, J. Rapaport, Ole Hansen, S. T. Scott, F. T. Dao, J. O. Ottesen, J. L. Mikesell, and B. A. Wolff)

\section{The $\mathrm{Ba}^{138}(\mathrm{~d}, \mathrm{p}) \mathrm{Ba}^{139}$ Reaction}

The $\mathrm{Ba}^{138}(\mathrm{~d}, \mathrm{p}) \mathrm{Ba}^{139}$ reaction has been studied using the multiple-gap spectrograph at a deuteron bombarding energy of $7.5 \mathrm{MeV}$. The data analysis has been completed and the results published in Physics Letters. ${ }^{2}$ However, it was considered important to continue work on this reaction and to check experimentally whether Coulomb stripping plays an important role in this

1. T.A.Belote, H.Y.Chen, Ole Hansen, and J. Rapaport,. Phys. Rev.142, No. 3, 624 (1966)

2. J. Rapaport and W. W. Buechner, Phys. Lett. 18, 299 (1965). 
case. Therefore, several new exposures at 5.0, 5.5,6.0,6.5,7.0, and 7.5 MeV incident deuteron energy have been made, and the data analysis is now in progress. (J. Rapaport, E. R. Chalbaud, H. A. White)

\section{The $\mathrm{Cl}^{37}(\mathrm{~d}, \mathrm{p}) \mathrm{Cl}^{38}$ Reaction}

The $(d, p)$ reaction from a target enriched in $\mathrm{Cl}^{37}$ has been studied with the multiple-gap spectrograph using a $7.5 \mathrm{-MeV}$ deuteron beam. A total of 37 states in $\mathrm{Cl}^{30}$ were identified up to an excitation energy of $5.5 \mathrm{MeV}$. All showed an angular distribution characteristic of a stripping process. The ground-state $\mathrm{Q}$-value was measured to be $3.878 \pm 0.010 \mathrm{MeV}$, in agreement with previous measurements. Transition strengths and values of the orbital angular momenta of the transferred neutrons were derived from the measurements by a zero-range, distorted-wave, Born-approximation analysis. These results have been submitted for publication in Nuclear Physics. (J. Rapaport and W. W. Buechner) .

\section{Optical-model Analyses and DWBA Calculations}

A number of deuteron and $\mathrm{He}^{3}$ elastic-scattering and stripping-reaction data were analyzed using the existing computer program codes ABACUS and JULIE. The optical-model parameters used in the DWBA calculations were derived from fitting the elastic-scattering data of the target nucleus at the appropriate bombarding energy. These calculations predicted with fair accuracy the positions and absolute magnitudes (and hence the spectroscopic factors) of the main peaks of the stripping angular distributions and also reproduced reasonably well the variation of peak cross sections with energy and $Q$-values.

The following reactions have been analyzed:

$$
\begin{array}{ll}
\mathrm{Ca}^{42,}{ }^{46}(\mathrm{~d}, \mathrm{p}) \mathrm{Ca}^{43,47} & \mathrm{~S}^{32}\left(\mathrm{He}^{3}, \mathrm{~d}\right) \mathrm{P}^{33} \\
\mathrm{Ni}^{58,60}(\mathrm{~d}, \mathrm{p}) \mathrm{Ni}^{59,61} & \mathrm{Ca}^{43,44}(\mathrm{~d}, \mathrm{p}) \mathrm{Ca}^{44,45} \\
\mathrm{Ca}^{40}\left(\mathrm{He}^{3}, \mathrm{~d}\right) \mathrm{Sc}^{41} & \mathrm{Cr}^{50}(\mathrm{~d}, \mathrm{p}) \mathrm{Cr}^{51} \\
\mathrm{Cr}^{52},{ }^{53}(\mathrm{~d}, \mathrm{p}) \mathrm{Cr}^{53,54} & \mathrm{Sr}^{88}(\mathrm{~d}, \mathrm{p}) \mathrm{Sr}^{89} \\
\mathrm{Te}^{128}(\mathrm{~d}, \mathrm{p}) \mathrm{Te}^{129} & \mathrm{~V}^{50}(\mathrm{~d}, \mathrm{p}) \mathrm{V}^{51}
\end{array}
$$

(H. Y. Chen)

\section{Optical Parameters for $\mathrm{He}^{3}$}

A series of experiments has been performed in an effort to study the variations with mass number and energy in optical parameters for $\mathrm{He}^{3}$ elastic scattering. $\mathrm{He}^{3++}$ ions were scattered elastically from $\mathrm{C}^{12}, \mathrm{O}^{16}, \mathrm{Mg}^{24}, \mathrm{~S}^{32}$, and $\mathrm{Ca}^{40}$ at incident energies of $10,12.4$, and $14 \mathrm{MeV}$. The 
angular distributions from the 10 - and $12.4-\mathrm{MeV}$ experiments were analyzed using the ABACUS code to find the set of optical-model parameters that produced the best fit to the data. In all cases parameters were obtained for both surface and volume absorption. For each nucleus, sets of parameters with real well depths in the neighborhood of $100 \mathrm{MeV}$ were found to give satisfactory agreement between theory and data. Further analysis will be undertaken in order to find the best fit parameters with real well depths near $150 \mathrm{MeV}$ for all three incident energies. (Mrs. Helen J: Young)

\section{The $\mathrm{N}^{14}(\mathrm{~d}, \mathrm{p}) \mathrm{N}^{15}$ Reaction}

Data concerning the $\mathrm{N}^{14}(\mathrm{~d}, \mathrm{p}) \mathrm{N}^{15}$ reaction, taken in 1962 by J. N. Hallock, were reanalyzed and were corrected to take into account the experimental circumstances at that time. In this way, angular distributions were obtained for the seventeen lowest excited states in $\mathrm{N}^{15}$. A DWBA analysis is in progress. (J. Kuperus)

\section{Analog-State Studies in the Strontium Isotopes}

The states in $\mathrm{Y}^{89}$ that are isobaric analog states of the low-lying levels in $\mathrm{Sr}^{89}$ are presently being studied." An excitation function for elastically scattered protons from 4.9 to $7.5 \mathrm{MeV}$ has been measured on $\mathrm{Sr}^{88}$, and four analog resonances have been seen. These resonances have been compared to conventional Breit-Wigner shapes, and the widths $\Gamma_{p}$ are being related to spectroscopic factors from the $\mathrm{Sr}^{88}(\mathrm{~d}, \mathrm{p}) \mathrm{Sr}^{89}$ reaction. The latter reaction has been investigated with the

aid of the multiple-gap spectrograph. Angular distributions from the inelastic $\mathrm{Sr}^{88}\left(\mathrm{p}, \mathrm{p}^{\prime}\right)$ reaction leading to the first $2^{+}$state in $\mathrm{Sr}^{88}$ have also been measured at energies on and off the analogstate resonance. Asymmetries about $90^{\circ}$ have been observed, indicating interference between the resonance amplitude and some other term. (E. R. Cosman)

\section{Stripping Analysis of the $\mathrm{Te}^{128}(\mathrm{~d}, \mathrm{p}) \mathrm{T} \mathrm{e}^{129}$ Reaction}

The reaction $\mathrm{Te}^{128}(\mathrm{~d}, \mathrm{p}) \mathrm{Te}^{129}$ has been investigated by bombarding an enriched $\mathrm{Te}^{128}$ target with 7.5 - MeV deuterons from the generator and analyzing the emitted protons with the aid of the multiple-gap spectrograph. A resolution of about 12 to $15 \mathrm{keV}$ was obtained. This enabled the identification of 39 excited states in $\mathrm{Te}^{129}$ in the energy range 0 to $3.4 \mathrm{MeV}$, including many that had not previously been observed or resolved. The $\mathrm{Q}$-value of the ground state was found to be $3.857 \pm 0.010 \mathrm{MeV}$. The angular distributions of ten relatively intense proton groups have thus far been analyzed by means of the $e^{D W B A}$ program JULIE. Three of the $l_{n}$ values thus obtained 
were in disagreement with previously reported results. 1

X. The Ground-State Transition in $\operatorname{Ag}^{107}(\mathrm{~d}, \mathrm{p}) \mathrm{Ag}^{108}$

The $(\mathrm{d}, \mathrm{p})$ reaction from $\mathrm{Ag}^{107}$ was investigated in this laboratory several years ago using the single-gap spectrograph at 20,50, and 130 degrees and was reported in a previous $P$ rogress Report (November 1964, p.44, by M. Mazari). The highest energy group reported in 1957, and assumed to be the ground-state group, corresponds to a $\mathrm{Q}$-value of $4.973 \mathrm{MeV}$. This value was incompatible with the results from $(n, \gamma)$ work ${ }^{2}$ and indicated that the group observed in the (d,p) reaction probably resulted from a transition to an excited state in $\mathrm{Ag}^{108}$. In an effort to resolve this discrepancy, the experiment was repeated with the multiple-gap spectrograph using a thicker target of more highly enriched $\mathrm{Ag}^{107}$ and five times more exposure. The results revealed an extremely weak proton group $78 \mathrm{keV}$ above the previously reported group (intensity 0.5 percent of first excited state at $37.5^{\circ}$ ). The new ground-state Q-value, 5. $051 \mathrm{MeV}$, is in agreement with the $(n, \gamma)$ results.

The scanning of the nuclear emulsions for protons corresponding to the excited states is currently under way. (A. Sperduto, M. Mazari (Mexico), D. N. Schramm, and M.A.M. Keehner)

\section{Collaboration with Other Universities}

During the period covered by this report, investigators from other universities have made use of the MIT-ONR generator and multiple-gap facility. Professor C. K. Bockelman from Yale University obtained exposures for angular-distribution studies of protons from 7.5-MeV deuteron bòmbardment of $\mathrm{Mn}^{55}$ and $\mathrm{Cd}^{113}$. Also obtained weré angular-distribution spectra of alpha particles and protons inelastically scattered from 7.5-MeV proton bombardment of $\mathrm{Ti}^{47}$ and $\mathrm{V}^{50}$.

A. Graue from the University of Bergen in Norway made exposures with $7.5-\mathrm{MeV}$ incident beams to obtain angular distributions of the $K^{41}(p, \alpha) A r^{38}, K^{d 1}(d, \alpha) A r^{39}$, and $\operatorname{Te}^{130}(d, p) T^{131}$ reactions.

Dr. B. Cohen from the University of Pittsburgh obtalned an angular-distribution exposure for the $\mathrm{Ge}^{70}(\mathrm{~d}, \mathrm{p}) \mathrm{Ge}^{71}$ reaction.

1. R. K. Jolly, Phys. Rev. 136, B 683 (1964).

2. G. A. Bartholomew and B. B. Kinsey, Can. J. Phys. 31, 1025 (1953). 
are reported elsewhere in this report and partial results were presented at the Gatlinburg Conference in Tennessee last November.

The energies of the levels in the even isotopes of chromium had not been intensively surveyed by means of the ( $\left.p, p^{\prime}\right)$ reaction. Only one level in each of $\mathrm{Cr}^{50}$ and $\mathrm{Cr}^{54}$ and five levels in $\mathrm{Cr}^{52}$ had previously been reported. Angular distribution of inelastic protons from enriched targets of each of the four stable isotopes of chromium have been obtained using 7.5-MeV incident protons. Only five spectra at $52.5,67.5,82.5,97.5$, and 120 degrees have thus far been analyzed in each case. The results of the energy levels measured are listed in Table 5.I.

Table 5.I

Energy Levels of the Chromium Isotopes from ( $\left.p, p^{\prime}\right)$ Reactions

\begin{tabular}{l}
$\begin{array}{l}\text { Level } \\
\text { Number }\end{array}$ \\
\hline 1 \\
2 \\
3 \\
4 \\
5 \\
6 \\
7 \\
8 \\
9 \\
10 \\
11 \\
12 \\
13 \\
14 \\
15 \\
16 \\
17 \\
18 \\
19 \\
20 \\
21 \\
22 \\
23 \\
24 \\
25 \\
26 \\
\end{tabular}

\begin{tabular}{r}
$\mathrm{Cr}^{50}$ \\
\hline 0.778 \\
1.875 \\
2.919 \\
3.156 \\
3.320 \\
3.590 \\
3.606 \\
3.621 \\
3.694 \\
3.788 \\
3.839 \\
3.869 \\
3.890 \\
3.929 \\
\end{tabular}

\begin{tabular}{c}
$\mathrm{Cr}^{52}$ \\
\hline 1.433 \\
2.368 \\
2.645 \\
2.765 \\
2.963 \\
3.113 \\
3.162 \\
3.417 \\
3.474 \\
3.614 \\
3.771 \\
3.947 \\
4.011 \\
4.036
\end{tabular}

\begin{tabular}{l}
$\mathrm{Cr}^{53}$ \\
\hline 0.559 \\
0.995 \\
1.278 \\
1.527 \\
1.964 \\
2.161 \\
2.222 \\
2.310 \\
2.442 \\
2.646 \\
2.661 \\
2.697 \\
2.761 \\
2.815 \\
2.980 \\
3.126 \\
3.172 \\
3.255 \\
3.339 \\
3.423 \\
3.590 \\
3.609 \\
3.685 \\
3.700 \\
3.824 \\
3.960
\end{tabular}

(A. Sperduto, N. M. Rao, E. R. Chalbaud, C. A. Wiedner (Heidelberg), and J. E. Robertshaw (P rovidence College).

$\sim$ XIV. New Spectrograph

A split-pole spectrograph ${ }^{1}$ for very high resolution studies is being designed. It will be mounted outside the vacuum tank of the multiple-gap spectrograph and operated in conjunction

1. H. A. Enge, Nucl. Instr, and Methods, 28, 119 (1964). 
ONR Generator Group

XII. Reaction Studies from the Nickel Isotopes

The $(d, p)$ and ' $\left(p, p^{\prime}\right)$ reactions from the nickel isotopes have been investigated in this laboratory during the past several years. Energy levels in all the isotopes and angular distributions from the $(d, p)$ reactions analyzed with the simple plane-wave theory have been reported in previous Progress Reports. With the recent success of the distorted-wave Born approximation (DWBA), a reanalysis of these data is being made using the code JULIE in an attempt to obtain self-consistent spectroscopic information. Additional bombardments on some of the nickel isotopes were made necessary in order to deduce absolute cross sections and to permit nurmalization of the older data. The first of these analyses, on the $\mathrm{Ni}^{58}(\mathrm{~d}, \mathrm{p}) \mathrm{Ni}^{59}$ reaction, is completed, and a manuscript for publication has been submitted to the Physical Review. The DW analyses of the $(d, p)$ angular distributions from $\mathrm{Ni}^{60}, \mathrm{Ni}^{61}, \mathrm{Ni}^{62}$, and $\mathrm{Ni}^{64}$ are currently under way.

In view of the recent successes in observing characteristic patterns in the angular distributions of $\left(d, d^{\prime}\right)$ reactions, a series of exposures recording the angular distribution of inelastic deuterons from all the nickel isotopes were made using $7.5-\mathrm{MeV}$ incident particles. A DWBA analysis is being attempted. The data from the $(d, p),\left(p, p^{\prime}\right)$, and $\left(d^{\prime} d^{\prime}\right)$ transitions populating the low-lying levels in $\mathrm{Ni}^{61}$ are being compared, and results may provide information on the single-particle and/or collective character of these states. (E. R. Cosman, A. Sperduto, G. Nair, H. A. Enge, A. Sparer, and B. J. O' Brien)

\section{Reaction Studies from the Chromium Isotopes}

Data analysis of the angular distribution of protons from the $(d, p)$ reactions on enriched isotopes of chromium is continuing. The analysis of the $\mathrm{Cr}^{52}(\mathrm{~d}, \mathrm{p}) \mathrm{Cr}^{53}$ and $\mathrm{Cr}^{53}(\mathrm{~d}, \mathrm{p}) \mathrm{Cr}^{54}$ reactions is completed, and the results are currently being prepared for publication. A preliminary analy sis of the $\mathrm{Cr}^{54}(\mathrm{~d}, \mathrm{p}) \mathrm{Cr}^{55}$ reaction for levels up to $3-\mathrm{MeV}$ excitation was reported at the Washington meeting of the American Physical Society in April 1965. Poor resolution in the region of higher excitation in $\mathrm{Cr}^{55}$, resulting from machine difficulties encountered during the course of this experiment, prevents adequate analysis of levels above $3.0 \mathrm{MeV}$. A repeat of this experiment to study the region above $3.0 \mathrm{MeV}$ has been made.

A study of the $\mathrm{Cr}^{50}(\mathrm{~d}, \mathrm{p}) \mathrm{Cr}^{51}$ reaction had first been undertaken using $6.0-\mathrm{MeV}$ incident deuterons at a time when generator difficulties prevented the use of higher energies. (See, November 1964 Progress Report, p. 95). The majority of the nuclear emulsions from this experiment has been scanned in Heidelberg (Germany), and the data analysis is well under way. More recently, when $\left(d, d^{\prime}\right)$ and $\left(p ; p^{\prime}\right)$ experiments (see below) at higher energies on the chromium isotopes were being performed, the $\mathrm{Cr}^{50}(\mathrm{~d}, \mathrm{p}) \mathrm{Cr}^{51}$ reaction was repeated using $7.5-\mathrm{MeV}$ deuterons in order to permit better comparison with the stripping analysis of the $(d, p)$ reactions on the other chromium isotopes. The emulsions from this experiment are being scanned and the data analyzed at Providence College (Rhode Island). The work on the (d, d') reactions on $\mathrm{Cr}^{50}$ and $\mathrm{Cr}^{52}$ 
with the latter. Particles from the multiple-gap targets can reach this new spectrograph through a hole in the yoke at 108.75 degrees. Because of the long flight path to the new spectrograph (4 meters), the solid angle accepted will be small; that is, of the same order of magnitude as the multiple-gap spectrograph, in spite of transverse-plane focusing. The long object distance results in a very small magnification of the spectrograph, which again means that a fairly large uncertainty in beam position and beam size from the target can be tolerated without seriously affecting the resolving power. The new spectrograph will have $\mathrm{Mu}$-metal pole pieces for attaining maximum accuracy and resolving power. (H. A. Enge, J. E. Spencer and R. L. Kusper)

XV. Other $(d, p)$ and $\left(d, d^{\prime}\right)$ Reactions on Titanium Isotopes

A collaboration between Yale University, the University of Copenhagen (Niels Bohr Institute), and M.I.T. on (,$p)$ and $\left(d, d^{\prime}\right)$ reactions on titanium isotopes has been brought to a conclusion. In addition to the reactions on titanium mentioned previously, papers on the $\mathrm{Ti}^{50}(\mathrm{~d}, \mathrm{p}) \mathrm{Ti}^{51}$ reaction ${ }^{1}$, the $\mathrm{Ti}^{47}(\mathrm{~d}, \mathrm{p}) \mathrm{Ti}^{48}$ reaction ${ }^{2}$, the $\mathrm{Ti}^{49}(\mathrm{~d}, \mathrm{p}) \mathrm{Ti}^{50}$ reaction ${ }^{3}$ have been published. The data analysis of the $\mathrm{Ti}^{48} \cdot(\mathrm{d}, \mathrm{p}) \mathrm{Ti}^{49}$ reaction has been concl uded. (A. Sperduto and Ole Hansen)

\section{Reactions on the Zinc Isotopes}

A program has been started to study single-particle and collective states of the isotopes of zinc up to several $\mathrm{MeV}$ excitation. Of particular interest is the study of the $(d, p)$ and $\left(d, d^{\prime}\right)$ transitions to those states that are first-order forbidden in the $(d, p)$ stripping process.

Angular distributions have been taken for $\mathrm{Zn}^{66}(\mathrm{~d}, \mathrm{p}) \mathrm{Zn}^{67^{*}}, \mathrm{Zn}^{66}\left(\mathrm{~d}, \mathrm{~d}^{*}\right) \mathrm{Zn}^{66^{*}}, \mathrm{Zn}^{67}\left(\mathrm{~d}, \mathrm{~d}^{\prime}\right) \mathrm{Zn}^{67^{*}}$, $\mathrm{Zn}^{67}(\mathrm{~d}, \mathrm{p}) \mathrm{Zn}^{68^{*}}$, and $\mathrm{Zn}^{6}{ }^{6}(\mathrm{~d}, \mathrm{~d})$ elastic at $7.5 \mathrm{-MeV}$ deuteron energy. $\dot{A}$ considerable portion of the scanning on the $\mathrm{Zn}^{67}\left(d, d^{\prime}\right), \mathrm{Zn}^{66}(\mathrm{~d}, \mathrm{p})$, and $\mathrm{Zn}^{66}\left(\mathrm{~d}, \mathrm{~d}^{\prime}\right)$ has been completed, both here and at the University of Pittsburgh. Further scanning and analysis of the data will be continued here and at the Niels. Bohr Institute in Copenhagen. Targets are available for $\mathrm{Zn}^{64}, \mathrm{Zn}^{66}, \mathrm{Zn}^{67}$, and $\mathrm{Zn}^{68}$; further $(d, p)$ and $\left(d, d^{\prime}\right)$ angular distributions and $\left(p, p^{\prime}\right)$ spectra are planned to complete the program. (W. H. Moore, Ole Hansen (Copenhagen), and D. J. Pullen (Pennsylvania)).

1. P.D.Barnes, C.K.Bockelman (Yale), Ole Hansen (Copenhagen), and A. Sperduto, Phys. Rev. 136, B 438 (1964).

2. P.D.Barnes and C.K.Bockelman (Yale), Ole Hansen (Copenhagen), and A. Sperduto, Phys. Rev. 138, B 13 (1965).

3. P.D. Barnes and C.K.Bockelman (Yale), Ole Hansen, and A. Sperduto, Phys. Rev. 140, B 42 (1965). 


\section{PUB LICATIONS}

T. A. Belote, J. H. B jerregaard, Ole Hansen, and G. R. Satchler, "Levels in ${ }^{43} \mathrm{Ca}$ and ${ }^{46} \mathrm{Ca}$ Studied by the ${ }^{43} \mathrm{Ca}\left(d, d^{\prime}\right),{ }^{46} \mathrm{Ca}\left(d, d^{\prime}\right)$, and ${ }^{46} \mathrm{Ca}\left(p, p^{\prime}\right)$ Reactions", Phys. Rev. 138 , B 1067 (1965).

T. A. Belote, W. E. Dorenbusch, Ole Hansen, and A. Sperduto, "Energy Levels in ${ }^{48} \mathrm{Ti}$ and ${ }^{50} \mathrm{Ti}$ Observed from Inelastic P roton Scattering at $7 \mathrm{MeV}^{\prime \prime}$, P hys. Lett. 14, 323 (1965).

T. A. Belote, A. Sperduto, and W. W. Buechner, "The Energy-level Structure of Ca ${ }^{41}$ from the $\mathrm{Ca}^{40}(\mathrm{~d}, \mathrm{p}) \mathrm{Ca}^{41}$ Reaction", Phys. Rev. 139, B 80 (1965).

T. A. Belote, W. E. Dorenbusch, Ole Hansen, and J. Rapaport, "Angular Distributions from Shell-model Forbidden (d,p) Transitions to Spin $5 / 2$ States in $\mathrm{Ca}^{43}, \mathrm{Ca}^{45}$, and $\mathrm{Ti}^{477 "}$ Nucl. Phys. 73, 321 (1965).

P. D. Barnes and C. K. Bockelman (Yale), Ole Hansen (Copenhagen), and A. Sperduto, "Inelastic Deuteron Scattering and $(d, p)$ Reactions from Isotopes of Titanium (II): $\mathrm{Ti}^{47}(\mathrm{~d}, \mathrm{p}) \mathrm{Ti}^{48 *}$ Phys. Rev. 138, B13 (1965).

P. D. Barnes and C. K. Bockelman (Yale), Ole Hansen, and A. Sperduto: "Inelastic Deuteron Scattering and $(d, p)$ Reactions from Isotopes of Titanium III: $\mathrm{Ti}^{49}(d, p) \mathrm{Ti}^{50}$ ", Phys. Rev. 140, B 42 (1965).

J. Rapaport and W. W. Buechner, "Stripping Analysis of the ${ }^{138} \mathrm{Ba}(\mathrm{d}, \mathrm{p})^{139} \mathrm{Ba}$ Reactions", Phys. Lett. 18, 299 (1965).

H. A. Enge, "Converting an Ion-Optical Layout into the Design of a P ractical Magnet System," $P$ roc. International Symposium on Magnet Technology 84 (1965).

\section{ADDRESSES}

Ole Hansen, "Nuclear Structure Studies with $(t, p)$ Reactions"

B rookhaven National Laboratory, December 1, 1964;

Princeton University, December 2, 1964.

Yale University, February 15, 1965;

Columbia University, March 12, 1965;

Chalk River, April 16, 1965.

H. A. Enge, "Nuclear Spectroscopy with B road-range Magnetic Spectrography, Present and Future", Seminar, Michigan State University, October 26, 1965.

T. A. Belote, "Nuclear Spectroscopy in the $f_{7} / 2$ Shell", Seminar, Oak Ridge National Laboratory, October 22, 1965.

T. A. Belote, "Level Structure of the Odd Calcium Isotopes", Colloquium, MIT, December 7, 1965.

\section{ABSTRACTS}

Ole Hansen, T. A. Belote, J. H. B jerregaard (Copenhagen), and G. R. Satchler (Oak Ridge), "Inelastic Scattering of Deuterons and Protons from $\mathrm{Ca}^{46} "$, Bull. Am. Phys. Soc. 10,104 (1965).

P. D. Barnes and C. K. Bockelman (Yale), Ole Hansen, and A. Sperduto, "Ti ${ }^{50}$ Levels Excited by the $\mathrm{Ti}^{49}(\mathrm{~d}, \mathrm{p})$ Reaction", Bull. Am. Phys. Soc. 10, 39 (1965).

W. E. Dorenbusch, T. A. Belote, and Ole Hansen, "Energy Levels in Ti ${ }^{48}$ Observed in Inelastic P roton Scattering", Bull. Am. P hys. Soc. 10, 104 (1965).

D. J. Pullen, A. Sperduto, and E. Kashy (Michigan State), "Angular-distribution Measurements from $\mathrm{Ne}^{20}(\mathrm{~d}, \mathrm{p}) \mathrm{Ne}^{21}$ and $\mathrm{Ne}^{22}(\mathrm{~d}, \mathrm{p}) \mathrm{Ne}^{23 "}, \mathrm{~B}$ ull. Am. P hys. Soc. 10, 38 (1965).

T. A. Belote, J. Rapaport (Chile), and H. Y. Chen, "Deuteron Stripping from Ca ${ }^{46 ", ~ B u l l . ~ A m . ~}$ Phys. Soc. 10, 25 (1965).

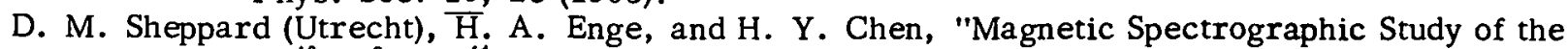
$\mathrm{Ca}^{40}\left(\mathrm{He}^{3}, \mathrm{~d}\right) \mathrm{Sc}^{41}$ Reaction", Bull. Am. Phys. Soc. 10, 25 (1965).

J. Rapaport (Chile), A. Sperduto, and W. W. Buechner, "Stripping Analysis of the $\mathrm{Ti}^{46}(\mathrm{~d}, \mathrm{p}) \mathrm{Ti}^{47}$ Reactions", Bull. Am. Phys. Soc. 10, 511 (1965). 
(Mrs.) H. J. Young, H. A. Enge, and H. Y. Chen, "Elastic Scattering of $\mathrm{He}^{3}$ by Nuclei in the Light-to-Middle Mass Region, "Bull. Am. Phys. Soc. 10, 539 (1965).

T. A. Belote, W. E. Dorenbusch, and Ole Hansen, "Inelastic Scattering of 7.5-MeV Deuterons from Ca44", Bull. Am. Phys. Soc. 10, 539 (1965).

W. E. Dorenbusch, T. A. Belote, and Ole Hansen, "Inelastic Scattering of 7.5-MeV Deuterons from $\mathrm{Ca}^{42 ",}$ Bull. Am. Phys. Soc. 10, 539 (1965).

C. A. Wiedner (Heidelberg), A. Sperduto, H. A. Enge, and W. W. Buechner, "Stripping Analysis of the $\operatorname{Cr}^{54}(d, p) \operatorname{Cr}^{55}$ Reactions, "Bull. Am. P hys. Soc. 10, 512 (1965).

\section{THESES}

(Mrs.) Helen J. Young, "An Experimental Stúdy of Optical-Model Parameters for $\mathrm{He}^{3}$ Elastic Scattering", M.S. (June 1965).

M. A. Skerry, "A Study of the Energy Levels of $\mathrm{Sn}^{119}$ through (d,p) Reactions on $\mathrm{Sn}^{118}$ ", B.S., (June 1965)

A. Sparer, Angular Distribution of P rotons Inelastically Scattered from $\mathrm{Ni}^{61 "}$ 1" B.S. (June 1965).

S. T. Scott, "Excited Levels of $\mathrm{Ti}^{48}$ seen in the $\mathrm{V}^{50}(\mathrm{~d}, \alpha) \mathrm{Ti}^{48}$ Reaction", B.S. (June 1965).

M. A. M. Keehner, "The Ground State and Angular Distributions of Ag108", B.S. (June 1965).

J. A. Ottesen, "Ca ${ }^{42}(\mathrm{~d}, \mathrm{p}) \mathrm{Ca}^{43}$ and Rutherford Slit Calibration", B.S. (June 1965).

B. J. O'Brien, "Investigation of the Stripping Reaction $\mathrm{Ni}^{64}(\mathrm{~d}, \mathrm{p}) \mathrm{Ni}^{65 "}, \mathrm{M} . \mathrm{S}$. (August 1965). 


\section{RADIOACTIVITY GROUP \\ RADIOACTH}

\section{General Research Topics}

A. Measurement of Magnetic and Quadrupole Moments of Short-lived Nuclear States using Angular Correlation and Mossbauer Technique. $\gamma-\gamma$ Angular Correlation.

Precession of correlations through nuclear intermediate states are measured in either external field of about 20,000 gauss (for lifetimes $>10^{-10} \mathrm{sec}$ ) or in internal hyperfine fields (up to $10^{6}$ gauss) for lifetimes $<10^{-10} \mathrm{sec}$.

Systematic magnetic moments for short-lived $\left(\sim 5 \times 10^{-11} \mathrm{sec}\right) 2+$ state in $\mathrm{Pt}^{192}, \mathrm{Pt}^{194}$, and $\mathrm{P} \mathrm{t}^{196}$ have been obtained by using the large internal field acting on $\mathrm{Pt}$ nuclel in ferromagnetic iron dilute Pt alloys. The field has been measured by observation of Mossbauer splitting of $99 \mathrm{keV}$ transition of the odd isotope $\mathrm{Pt}^{195}$ in a Fe-Pt alloy.

Experiments extending this technique to even shorter lives are planned in order to measure for the first time moments of some interesting collective vibrational states, e.g., the octupole vibration 3 - states (as in $\mathrm{Pb}^{208}$ ) and the 2+ vibrational states in spherical nuclei (cadmium, tellurium).

The angular distribution of MUssbauer scattered radiation, for transitions in Os and $W$ isotopes, has been measured. Particularly large effects have been observed in $\mathrm{Os}^{186}$. The precession in external fields has been measured leading to independent values for moments of $2+$ rotational states.

Mơssbauer absorption measurements are being extended to several rare earth (and other) elements in different materials, in order to determine hyperfine interactions. Attempts are made to correlate changes in mean square charge radius of nuclear states within a rotational band with the rotation vibration interaction.

B. Nuclear Spectroscopy using the New High Resolution Germanium Gamma-Counters

This tool is being actively explored and applied to a variety of uses, and, by virtue of high resolution, has also suggested some new problems, on which work has begun -- such as detection of doppler-shifted gamma -rays for lifetime measurements, resolution of theoretically expected doublet structures, because of improved signal to background.

\section{Some Detailed Results}

\section{A. Alpha-Gamma Delayed Coincidence Lifetime Measurements}

Using a silicon surface barrier alpha detector and a lead-loaded $(5 \%)$ plastic scintillator gamma detector, with a conventional fast-slow coincidence spectrometer, lifetimes of excited states populated by alpha decay in the Actinium 227 decay chain are being studied. 
For the $286.1 \mathrm{keV}$ state in $\mathrm{Ra}^{223}$ populated by a $21 \%$ alpha branch of $5.755 \mathrm{MeV}$ from $\mathrm{Th}^{227}$, preliminary measurements give:

$$
\mathrm{T}_{1} / 2=0.56 \times 10^{-9} \mathrm{sec} .
$$

Previous measurements, ${ }^{1}$ using a centroid shift méthod reported a value of $0.77 \mathrm{~ns}$.

For the $351 \mathrm{keV}$ state in $\mathrm{Tl}^{207}$ populated by a $17 \%$ alpha branch of $6.273 \mathrm{MeV}$ from $\mathrm{Bi}^{211}$,

$$
\mathrm{T}_{1} / 2<0.16, \times 10^{-9} \mathrm{sec} .
$$

The $445 \mathrm{keV}$ state in $\mathrm{Rn}^{219}$ which is de-excited by $\mathrm{M} 2$ radiation ${ }^{2}$ and has a single particle lifetime prediction of about 1 ns is currently being studied. Initial experiments indicate a life -: time of less than $1 \mathrm{~ns}$.

Alpha -energy resolution of about $50 \mathrm{keV}$. FWHM and a good knowledge of the decay schemes allow precise alpha channels to be set. Gamma channels are set by first gating the gamma singles spectrum with the selected alpha channel in coincidence with all fast alpha-gamma coincidences. The gamma window was then set, near the high end, on $15-20 \%$ of the approximate continuum spectrum observed.

Fast signals are taken from a fast rise time preserving amplifier on the alpha side and through an emitter follower at a photomultiplier (56 AVP) dynode, into Chronetics fast discriminators and a time to pulse height converter. The time spectrum gated by slow coincidences is analyzed and lifetime slopes measured. Calibrations were made using standard cables with coincident inputs into the time to height converter. (S. G. Cohen, D. Murnick, and W. C. Schick)

B. The Investigation of the Magnetic Moments of First $2^{+}$States in Even-Even Osmium and Tungsten Isotopes

The following is the abstract of $\mathrm{Y}$. $-\mathrm{W}$. Chow's Ph.D. thesis, submitted to the Department of Physics on May 14, 1965.

Two experimental methods for measuring the magnetic moments of nuclear excited states with short lifetime $\left(10^{-9} \mathrm{sec}\right)$ namely, the integral reversed field method of perturbed angular distribution of the Mossbauer scattering radiation (IRFMS) and the measurement of the hyperfine splitting using Mossbauer effect (HS) are described. The IRFMS technique is unique in the sense that there are no recoil, no radiactivity effects and no radiation damage effects. The magnetic moments of first $2^{+}$states of even-even tungsten isotopes and osmium isotopes measured by these two methods are listed in Table 6.I.

1. H. Vartapetian, Comptes Rendus 246, 1680 (1958).

2. R. C. Pilger, Ph.D. Thesis, UCRL-3877, Berkeley (1957). 
Table 6.I

Magnetic Moments of First $2^{+}$States in Even-Even

Tungsten and Osmium Isotopes

\begin{tabular}{|c|c|c|c|c|c|}
\hline Isotope & $\begin{array}{c}\text { Magnetic } \\
\text { Moment nm } \\
\end{array}$ & g-factor & $\begin{array}{c}\text { Energy of the } \\
\text { level keV }\end{array}$ & $\begin{array}{l}\text { Half life } \\
\times 10^{-9} \mathrm{sec} \\
\end{array}$ & Technique \\
\hline $\mathrm{W}^{182}$ & $.465 \pm .054$ & $.233 \pm .027$ & 100 & 1.36 & IRFMS \\
\hline$W^{184}$ & $.5 \pm .052$ & $.25 \pm .026$ & 111 & 1.28 & (HS) \\
\hline $\mathrm{W}^{186}$ & $.676 \pm .065$ & $.34 \pm .033$ & 122 & 1.15 & IRFMS \\
\hline Os $s^{186}$ & $.636 \pm .022$ & $.318 \pm .011$ & 137 & .84 & IRFMS \\
\hline Os ${ }^{188}$ & $.63 \pm .066$ & $.315 \pm .033$ & 155 & .73 & IRFMS \\
\hline
\end{tabular}

The internal magnetic fields of tungsten iron alloy at the tungsten nucleus and of osmium iron alloy at the osmium nucleus were found to be $775 \pm 82$ Kgauss and $1330 \pm 140$ Kgauss, res pectively.

The ratio of the electric quadrupole moments of $\mathrm{W}^{184}$ to $\mathrm{W}^{182}$ is $\mathrm{Q}(184) / \mathrm{Q}(182)=1.0 \pm .12$ compared to $.95 \pm .15$ from coulomb excitation. (Y. $-W$. Chow and L. Grodzins)

\section{Liquid Helium Cryostat for MOssbauer Scattering Experiments}

A simply constructed cryostat designed for Mossbauer $\gamma$-ray scattering experiments is described. The sample volume is part of the transfer line from a liquid helium reservoir to a mechanical pump; the sample is thus cooled by helium flow. The helium vapor exiting from the sample volume cools the transfer line and radiation shield. The temperature of the sample is controlled by the helium flow rate determined by a needle valve at the pump. The operating range is from 9 to $\sim 300^{\circ} \mathrm{K}$. The liquid helium consumption in liters $\hbar$ is 0.3 at $10^{\circ} \mathrm{K}, 0.2$ at $16^{\circ} \mathrm{K}$, and 0.1 at $34^{\circ} \mathrm{K}$. (P. H. Barrett and L. Grodzins)

D. Mossbauer Scattering: The Quadrupole Interaction in Osmium Metal

In Mossbauer scattering of the $137-\mathrm{keV}$ gamma radiation from the first $2+$ state of $\mathrm{Os}^{186}$, the effect, i.e., the M४ssbauer intensity-to-background ratio at zero relative velocity between source and scatterer, is, at $15^{\circ} \mathrm{K}$, approximately $3: 1$. The full width at half-maximum of the velocity spectrum is $1.36 \pm 0.07$ times that theoretically expected. The Debye temperature of the osmium scatterer was determined and appears to increase with increasing temperature, from 4.2 to $80^{\circ} \mathrm{K}$. The average value of $\theta_{\mathrm{D}}$, assuming it to be the same for source and scatterer, is $375 \pm 20^{\circ} \mathrm{K}$. The angular distribution of the scattered radiation was measured using a three -: detector geometry. Assuming that the attenuation of the correlation is due to an axially symmetric electric field gradient ( $E F G$ ) acting on the quadrupole moment of the $2+$ state, we obtain for the quadrupole interaction, $\omega_{E}=(35.6 \pm 3.3) \times 10^{6} / \mathrm{sec}$. From the $B(E 2)$ value of the transition, the value the EFG of hexagonal close-packed (hcp) osmium is deduced to be $q=(3.50 \pm 0.50) \times 10^{17} \mathrm{~V} / \mathrm{cm}^{2}$. 
By comparing this value with the lattice contribution to the EFG as well as to the known EFG of hcp rhenium metal, we conclude that the conduction-electron contribution is significant and probably changes substantially from rhenium to osmium. (L. Grodzins and.Y. -W. Chow)

\section{E. Magnetic Moments of $2+$ States in Pt Nuclei}

From analysis of MUssbauer spectra taken with the $99 \mathrm{keV}$ gamma ray from the $3 / 2^{-}$ state of $\mathrm{Pt}^{195}$, using a $\mathrm{Au}^{195}$ in $\mathrm{Cu}$ source and a ferromagnetic Fe-Pt alloy absorber ( 3 at \% $\left.\mathrm{Pt}\right)$ :

$$
\begin{aligned}
& \mu\left(3 / 2^{\top}\right)=-.55 \pm .08 \mathrm{~nm} \\
& \mathrm{H}_{\text {int }}(\mathrm{Pt})=(1.34 \pm .07) \times 10^{6} \text { gauss. }
\end{aligned}
$$

From analysis of angular correlation experiments using $\operatorname{Ir}^{192}$ and $\operatorname{Ir}^{194}$ sources in ferromagnetic Ir -Fe alloy wire (3 at $\%$ Ir):

$$
\begin{array}{ll}
\mu(2+)=+.61 \pm .06 & 316 \mathrm{keV} \text { level of } \mathrm{Pt}^{192} \\
\mu(2+)=+.55 \pm .06 & 328 \mathrm{keV} \text { level of } \mathrm{Pt}^{194} \\
\mu(2+)=+.30 \pm .06 & 622 \mathrm{keV} \text { level of } \mathrm{Pt}^{194}
\end{array}
$$

$A \mathrm{Au}^{196}$ (carrier free) source in Fe wire gave:

$$
\begin{aligned}
& \mu(2+)=+.42 \pm .10 \quad 355 \mathrm{keV} \text { level of } \mathrm{Pt}^{196} \\
& \mathrm{H}_{\text {int }}(\mathrm{Pt})=(0.70 \pm .10) \times 10^{6} \text { gauss. }
\end{aligned}
$$

It is felt that the $\mathrm{Au}^{196}$, which was diffused into the $\mathrm{Fe}$ wire, may have gone along grain boundaries, accounting for the low field. (A. Buyrn and L. Grodzins)

F: Anisotropic MOssbauer Effect on Magnetic Hyperfine Splittings in Polycrystalline Materials.

Asymmetries in the intensities of components lines of quadrupole doublets have been observed in the recoil-free quadrupole spectra of some polycrystalline materials, particularly in some tin compounds. These asymmetries have been interpreted by Karyagin and Goldanski, et al. , as due to lattice anisotropy in the recoil-free fraction $f$. The present authors suggest that in general one also expects lattice anisotropies to influence the intensity of the lines observed in recoil -free spectra showing |magnetic hyperfine interactions (M.H.I.) for precisely the same physical reasons, namely the interplay between the angular variation of the $f$-factor and the angular distribution of the various components of the multipole electromagnetic radiation emitted between the various magnetic sub-states of the nuclear levels. A theoretical analysis of these effects for typical cases have beën completed. ('S. G. Cohen and P. Gielen - Dept. of Metallurgy)

\section{G. Evidence for Centrifugal Stretching in Deformed Nuclei}

The isomer shift which is proportional to the difference between the average radii of the ground and excited states has been measured for $W^{182,184, ~ 186 . ~ T h e ~ m e a s u r e m e n t s ~ p r o v e ~ t h a t ~ t h e ~}$ $0^{+}$and $2^{+}$members of the rotational bands in these isotopes have different r.m.s. radii. Cal culations indicate that centrifugal stretching (from the rotational-vibrational interaction) is probably important but anti-pairing effects which increase with the spin of the band member are 
also important. In $\mathrm{W}^{182}$ the value of $\frac{\Delta<\mathrm{R}^{2}>}{\mathrm{R}^{2}} \approx 1.3 \times 10^{-4}$. The data was obtained between $\mathrm{W}$ metal and $\mathrm{WCl}_{6}$ - Similar results have now been obtained with tungsten bronzes. Experiments are continuing on $\mathrm{Os}^{188}, \mathrm{Sm}^{152}$, and $\mathrm{Gd}^{154}$. (N. Blum, Y. -W. Chow, S. G. Cohen, L. Grodzins, R. Frankel, and D. Yeboah-Amankwah)

H. Gamma -Ray Decay Schemes of Europium -152, Europium-152m, and Europium -154

The nuclear energy-level spectra of $\mathrm{Sm}^{152}, \mathrm{Gd}^{152}$, and $\mathrm{Gd}^{154}$ have been studied from. the decay of $\mathrm{Eu}^{152}, \mathrm{E} \mathrm{u}^{152 \mathrm{~m}}$, and $\mathrm{Eu}{ }^{154}$ using $\mathrm{Ge}(\mathrm{Li})$ detectors. Coincidence measurements have been made with one $\mathrm{Ge}(\mathrm{Li})$ detector and one $\mathrm{NaI}$ detector. An efficiency curve has been obtained for a $\mathrm{Ge}(\mathrm{Li})$ detector that is accurate to about $5 \%$ between 250 and $1600 \mathrm{keV}$. Energies and intensities of some 80 gamma rays have been measured, about 30 of which are new. Two (and possibly three) new levels are added in $\mathrm{Sm}^{152}$, one in $\mathrm{Gd}^{152}$, and two in $\mathrm{Gd}^{154}$.

Reduced transition probability ratios have been calculated and compared with the predictions of the symmetric-rotor model with band mixing. A small amount of mixing between the gamma-vibrational and ground-state bands is found in $\mathrm{Sm}^{152}$ and $\mathrm{Gd}^{154}$. The four measured transition ratios from the gamma band in each isotope are consistent with a value of the mixing parameter $z_{\gamma}$ of .08. A similar value of $z_{\gamma}$ can be derived from the band-head energies and the Coulomb excitation data. The mixing between the beta-vibrational and ground-state bands is considerably stronger and consistent results are not obtained. K quantum numbers have been assigned to the odd -parity levels in $\mathrm{Sm}^{152}$ and $\mathrm{Gd}^{154}$. (W. E. Schick, Jr., H. Kraner, and A. R. Lewis)

\section{Internal Conversion Coefficient Measurements}

The internal conversion coefficients of transitions from the first excited level to the ground level in some even-even trans-lead elements are being studied. These are, uniformly, E2 transitions within the ground state rotational band. Semi-conductor $\alpha$ and $\mathrm{e}^{-}$detectors are being used and total conversion coefficients and $L: M+N+O$ ratios are desired. (R. Schlenker and R. Evans)

\section{J. Contoured Diffused-Junction Detectors}

1. For about a year we have been evaluating contoured diffused-junction silicon detectors ${ }^{1}$ which exhibit internal pulse amplification up to factors of about 100 for possible applications to nuclear spectroscopy and timing measurements. Several detectors have been kindly supplied by Mr. G. C. Huth of the General Electric Company, whose cooperation is greatly appreciated. An electrodeless nickel back $(\mathbf{n}+)$ contact has been substituted to lower the series resistance of the device and improve pulse rise time. (H. W. Kraner and W. R: Neal)

1. G. C. Huth, J. B. Trice, J. A. Shannon, and R. C. McKinney, I. E. E. E. Transactions on Nuclear Science, NS-12, 275 (Feb. 1965). 
2. Thus $\sim 70 \mathrm{keV}$ conversion electrons have been observed saturating a fast transis torized amplifier with the $\sim 5 \mathrm{~ns}$ system rise time and a pulse height equivalent to $\sim 5 \mathrm{MeV}$ energy loss. Non-saturating pulses from some diodes have had rise times as low as 5 to $10 \mathrm{nsec}$ and preliminary studies of fast timing capabilities have yielded prompt timing spectra with FWHM's on the order of $3 \mathrm{nsec}$ using the $5.77 \mathrm{MeV} \alpha$ of $\mathrm{Bi}^{212}$ (into semiconductor $\alpha$-detector) and the conversion electrons $(\sim 200 \mathrm{keV})$ of the second excited state of $\mathrm{Tr}^{208}$. (H. Kraner and W. R. Neal)

\section{K. Charge Sensitive FET P reamplifiers}

Work has been resumed on the charge-sensitive preamplifier utilizing FETS to obtain good energy resolution with solid state detectors. Energy resolutions of the order of $5 \mathrm{keV}$ (FWHM) have been achieved with a circuit which is currently being used in conjunction with a $\mathrm{Ge}(\mathrm{Li})$ detector, and has been in operation for almost a year. Circuits constructed since that time have not shown any significant improvement.

A cooled FET preamp was fabricated utilizing a Dewar using liquid nitrogen as the coolant. A method was devised to maintain the FET temperature at approximately $120^{\circ} \mathrm{C}$, and it was found that an improvement in room temperature resolution was effected.

It has been determined that a comparatively new Texas Instrument FET is superior to other recent types with respect to noise performance. Work is now continuing on a room temperature version incorporating this transistor. (A. R. Lewis, J. P. Morris, W. R. Neal)

\section{P UB LICATIONS}

P. H. Barrett and L. Grodzins, "Liquid Helium for M४ssbauer Scattering Experiments", Rev. Sci. Instr. 36, No. 11, 1607 (Nov. 1965).

N. Blum, A. J. Freeman, J. W. Shaner, L. Grodzins, "Mossbauer Studies of Spin Flop in Antiferromagnetic Hematite", J. Appl. Phys., Supplement 36, 1169 (April 1965).

Y. W. Chow, L. Grodzins, P. H. Barrett, "Mdssbauer Scattering II: The Gyromagnetic Moments of First $2+$ States in $W^{182}$ and $W^{186}$ and $O s^{186}$ and Os ${ }^{188 ", ~ P h y s . ~ R e v . ~ L e t t e r s ~} 15$, No. 8, 369 (Aug. 23, 1965).

L. Grodzins and J. Alonso, "Measurement of Total Attenuation Cross Sections in Aluminum and Gold for 14.4 keV Gamma Rays", Phys. Rev. 137, No. 3A, A975 (Feb. 1, 1965).

L. Grodzins, with M. Goldhaber and A. W. Sunyar, "Helicity of the Neutrino", in Alpha, Beta, and Gamma Ray Spectroscopy, (K. Siegbahn, ed.), North Holland, p. $\overline{1423-1431(1965)}$.

L. Grodzins and P. H. Stelson, "Nuclear Transition Probability, B (E2)", Nuclear Data 1, 21-102 (1965).

S. Ofer, E. Segal (Hebrew University, Jerusalem), I. Nowik (Israel A. E. C., Yavne, Israel), E. R. Bauminger, L. Grodzins (MIT), A. J. Freeman (Nat. Magnet Lab.), M. Schieber (Harvard), "Hyperfine Interactions in the Ground State and $22 \mathrm{keV}$ State of $\mathrm{Sm}^{149}$ in Ferromagnetic Compounds of Samarium", Phys. Rev. 137, A627 (January 1965).

W. Schick and L. Grodzins, "Spins and Parities of States in Gd ${ }^{152 ", ~ N u c l . ~ P h y s . ~ 62, ~} 254$ (March 1965). 


\section{THESES}

Y. W. Chow, "Investigation of the Magnetic Moments of the First 2+ States in Even-Even Osmium and Tungsten Isotopes", Ph.D. (June 1965).

J. A. Christensen, "A Test of Cold Argon Gas and Argon Liquid as Spark Chamber Working Substances", B.S. (May 1965).

S. H. Drake, "The Design, Construction, and Testing of a Set of Wide Gap Spark Chambers", B. S. (June 1965).

M. Efron, "The Energy Resolution of a Spark Chamber Total Absorption Cerenkov Counter Com bination" (with Dawn L. Friedell), B.S. (June 1965).

Dawn Lorelei Friedell, "The Energy Resolution of a Spark Chamber Total Absorption Cerenkov Counter Combination" (with M. Efron), B.S. (June 1965).

W. C. Schick, "Gamma-Ray Decay Schemes of Eu'152, Eu ${ }^{52 m}$, and Eu"154", Ph.D. (Nov. 1965).

M. D. Shuster, "An Attempt to Measure Forward Scattering Cross Sections in Al of $14.4 \mathrm{keV}$ Gamma-Radiation", B.S. (June 1965). 
I. Cyclotron Operation and Equipment

During the past half year the cyclotron has been given extensive use. Several $\left(\alpha, \alpha^{\prime}\right)$ and $(\alpha, \mathrm{p})$ experiments, an $\left(\alpha, \alpha^{\prime} \gamma\right)$, and an $(\alpha, 2 \alpha)$ experiment have been performed.

The beam optics have been further studied by first-order calculations and by experimental observations. Several new viewing ports were added in the vicinity of the first quadrupole pair. However, the performance of the cyclotron $\alpha$-particle beam has not been improved since last reported in the progress report; i.e., about 0.2 to $0.3 \mu \mathrm{a}$ of beam with an intrinsic overall resolution of 70 to $80 \mathrm{keV}$. To improve this we believe that improvements inside the cyclotron will have to be made. A new pump was installed to improve the cyclotron vacuum. We are also monitoring the stability of the cyclotron oscillator. Other improvements include the installation of antibacklash gears in the scattering chamber to improve angular accuracy.

During the summer the second position allowed by the $30^{\circ}$ analyzing magnet was developed.

- Fig. 7.1 shows a layout of the beam handling system as it now appears. The new side, labeled by SC2 has been used in the $(\alpha, 2 \alpha)$ and $\left(\alpha, \alpha^{\prime} \gamma\right)$ work. A new scattering chamber for $\gamma$ ray work was constructed for this purpose. Our improved knowledge of the beam optics proved useful in reducing the neutron and $\gamma$-ray background on this side.. We have also increased the amount of electronics we have so that both sides have their own systems with the exception of the multichannel analyzer. This has enabled us to use the cyclotron much more effectively. (F. Fay, E. White, H. Tannenbaum, W. J. Kossler, and A. Bernstein)

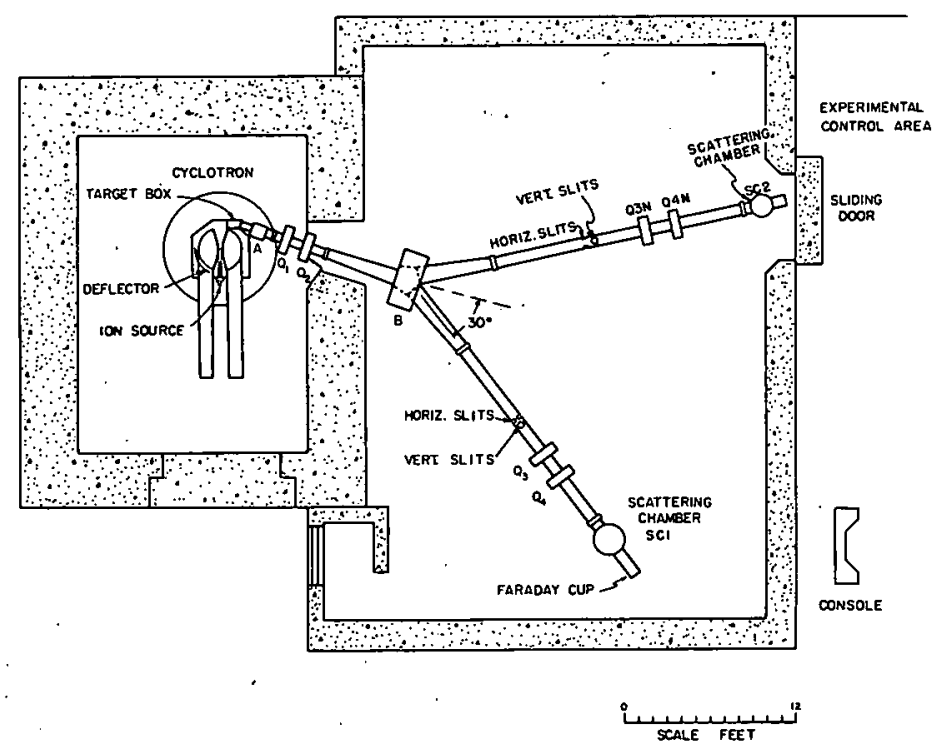

Figure 7.1

Layout of Cyclotron and Beam Handling System 


\section{II. $\mathrm{Ca}^{40}(\alpha, 2 \alpha) \mathrm{Ar}^{36}$ Reaction}

A further measurement on this reaction has been done at $30.8 \mathrm{MeV}$. One solid state detector was kept at $35^{\circ}$ from the beam axis while the other was swept in $5^{\circ}$-steps from $35^{\circ}$ on the other side of the beam to $60^{\circ}$ and then from $35^{\circ}+180^{\circ}$ to $60^{\circ}+180^{\circ}$. The purpose of this measurement is to see whether Bohr's theorem ${ }^{1}$ holds for the intermediate state previously observed. The theorem states that if the intermediate state has unique parity then the angular correlation of the decay from this state will repeat after $180^{\circ}$ in the center of mass coordinate system. These angular measurements were done both with and without counter telescope particle identification on both arms. For particle identification a multiplier circuit was constructed following Radeka's design. ${ }^{2}$ As opposed to the previous measurement all measurements here . were done on one target approximately $1 \mathrm{mg} / \mathrm{cm}^{2}$ thick. The solid angle of each detection was about 4 times greater than previously, thus allowing much faster collection of data. The results obtained with the $1 \mathrm{mg} / \mathrm{cm}^{2}$-thick target and another target approximately twice as thick were compared to see whether there was any unexpected degrading of the out-going alpha particles in the target. No such effect was observed.

At present the data are being transferred from paper tape to magnetic tape, prior to analysis. Preliminary results indicate that the larger number of low energy particles observed previously were protons, while the particles whose kinematics correspond to the $\mathrm{Ca}^{40}(\alpha, 2 \alpha) \mathrm{Ar}^{36}$ first-excited state are indeed alpha particles. (W. Kossler and M. Slade)

\section{Elastic and Inelastic Scattering of Alpha Particles}

\section{A. Ca Isotopes}

The scattering of alpha particles was measured on $\mathrm{Ca}^{42}, \mathrm{Ca}^{44}$, and $\mathrm{Ca}^{48}$. In addition, the previous measurement of $\mathrm{Ca}^{40}$ was repeated with better resolution and better statistics. Elastic data were taken at angles between $14^{\circ}$ and $166^{\circ}$ at intervals of $1.8^{\circ}$. Inelastic da ta were taken between $14^{\circ}$ and $65^{\circ}$, with data on a few of the strònger inelastic states present at larger angles. The analysis of this data is still in progress. Figure 7.2 shows a $\mathrm{Ca}^{42}$ spectrum at $30^{\circ}(\mathrm{lab})$ and an energy level diagram. Spins and parities of the levels above the $6^{+}$state have been assigned by us on the basis of the shapes of the differential cross sections. This angle favors negative parity states of $\mathrm{Ca}^{42}$. In particular we note the discovery of $3^{-}$states at $3.44,4.70$, and 4.98 $\mathrm{MeV}$ and a $5^{-}$state at $4.10 \mathrm{MeV}$. Figure 7.3 shows the scattering to the two known $2^{+}$states at 1.52 and $2.42 \mathrm{MeV}$. The solid lines represent DWBA fits with $\beta_{2}=0.17$ and 0.06 respectively. It is interesting that the much debated $2.42 \mathrm{MeV}$ states is 10 times weaker than the $1.52 \mathrm{MeV}$ state. Scattering to the $4^{+}$state at 2.75 is fit by the DWBA theory. The $6^{+}$state at $3.19 \mathrm{MeV}$ is

1. A. Bohr, Nucl. Phys. 10, 486 (1959)

2. V. Radeka, Fast Analogue Multipliers with Field-Effect Transistors, BNL $\mathbf{7 4 4 8 .}$ 
weak. The $0^{+}$state at $1.84 \mathrm{MeV}$ is excited; and a cross section will be presented in the future.

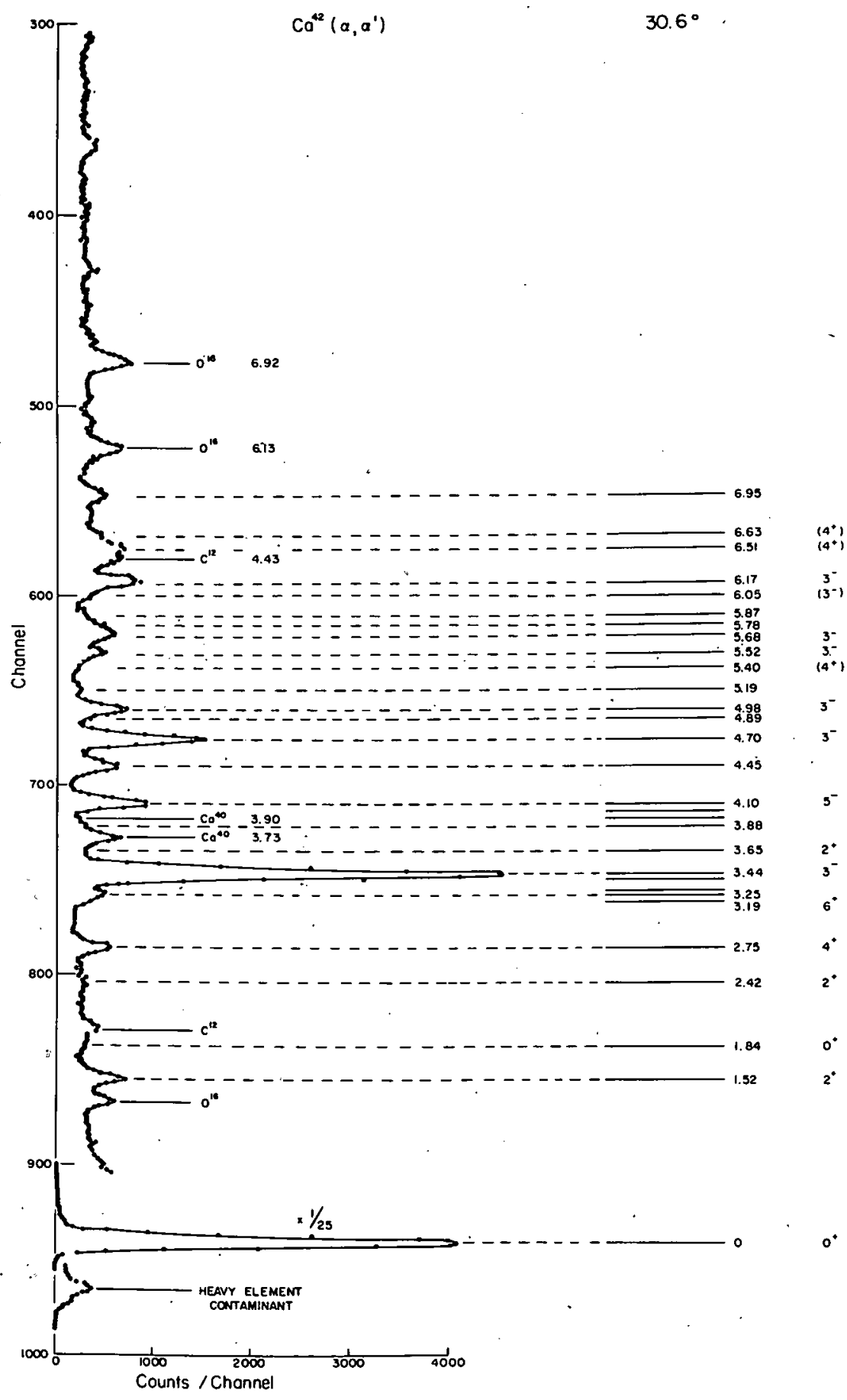

Figure 7.2

Spectrum of inelastic scattering of alpha particles from $\mathrm{Ca}^{42}$ at $30.6^{\circ}$ (lab) with an energy level diagram. This angle is favorable for negative parity states. 
Cyclotron Group

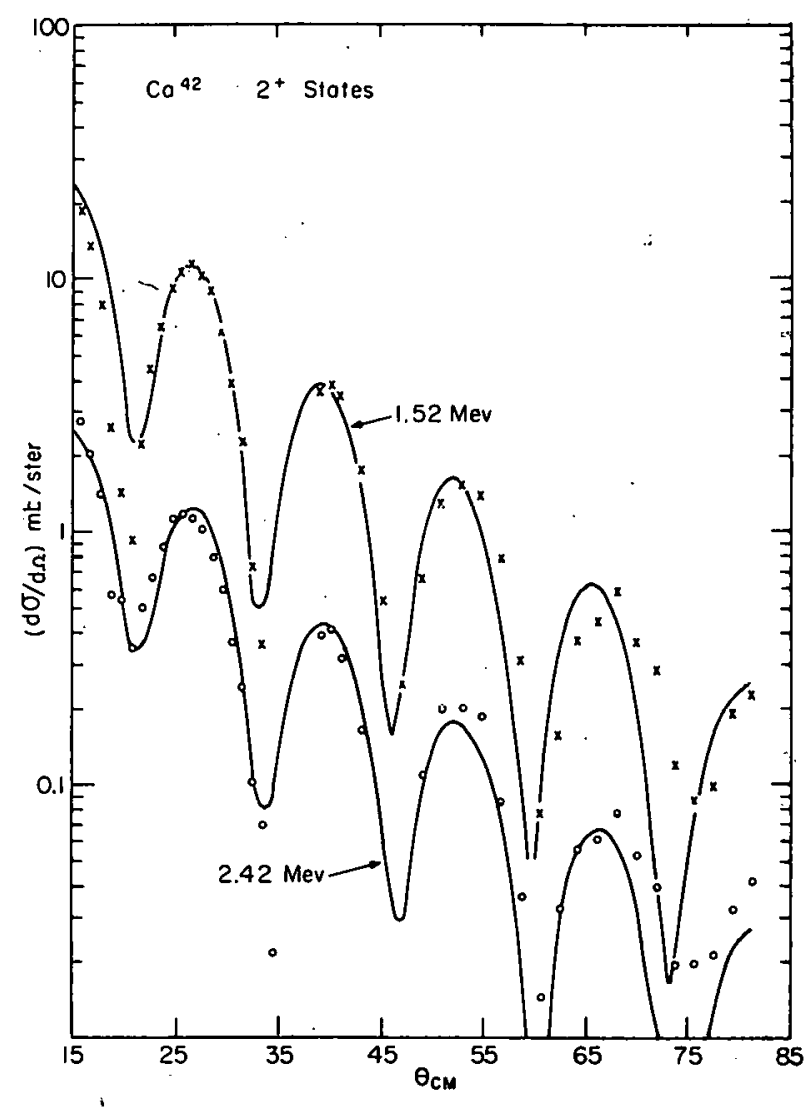

Figure 7.3

Differential cross section for the inelastic scattering of alpha particles to the $2^{+}$states of $\mathrm{Ca}^{42}$ at 1.52 and

2.42 MeV. The solid lines are DWBA fits.

Figure 7.4 shows a comparison of the strongly excited states of $\mathrm{Ca}^{40}$ and $\mathrm{Ca}^{48}$. In $\mathrm{Ca}^{48}$ we found $3^{-}$states at $4.50,5.37$, and $7.68 \mathrm{MeV}$, and a $5^{-}$state at $5.73 \mathrm{MeV}$. Both nuclei show the characteristics of a closed shell structure -- an absence of low lying states. From a variety of nuclear structure information it is becoming clear that $\mathrm{Ca}^{48}$ is a better "core" in the shell model sense than $\mathrm{Ca}^{40}$. It is interesting to look for evidence for this by examining the differences in the excited states of these nuclei. One notices the lowest excited state in $\mathrm{Ca}^{40}$ is $0^{+}$, while in $\mathrm{Ca}^{48}$ it is $2^{+}$. Furthermore there is the possibility of a positive parity rotational band in $\mathrm{Ca}^{40}$, composed of the levels at $3.35 \mathrm{MeV}\left(0^{+}\right), 3.90 \mathrm{MeV}\left(2^{+}\right)$, and $5.27 \mathrm{MeV}\left(4^{+}\right)$which fit an I $(I+1)$ sequence. At the present time we have no such candidates in $\mathrm{Ca}^{48}$. 


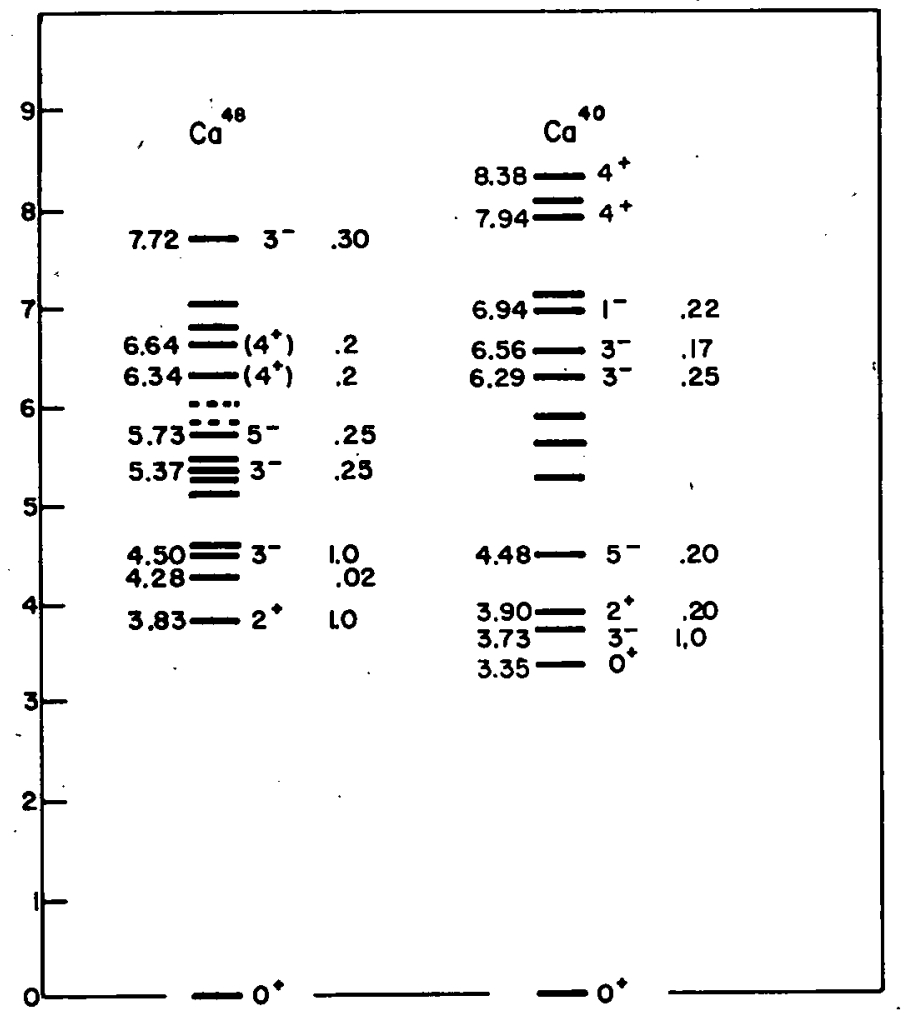

Figure 7.4

A comparison of the states in $\mathrm{Ca}^{40}$ and $\mathrm{Ca}^{48}$ which have been strongly excited by the $\left(\alpha, \alpha^{\prime}\right)$ reaction. These results do not show the full complexity of the spectra, particularly above $6 \mathrm{MeV}$ excitation. The numbers on the right side of each spectrum indicate relative cross sections in each isotope.

We believe that the most interesting results are the large number of $3^{-}$and $5^{-}$states that we have found. Table 7.I gives a summary of the states. Many of these assignments have been confirmed at the University of Washington. ${ }^{1}$ Here we also present the results for $\mathrm{Ca}^{44}$ which have not been previously discussed. We also show the angular distributions to some of these states in Figs. 7.5 and 7.6. Note the degree of similarity of the shapes of the curves for the different isotopes, and the large decrease in magnitude in going.from $\mathrm{Ca}^{40}$ to $\mathrm{Ca}^{48}$. It seems surprising that both the $3^{-}$and $5^{-}$,states decrease by about the same magnitude. This decrease is given numerically in Table 7.I. Here we have taken the ratios of the cross sections at their maximum at $32^{\circ}$, setting $\mathrm{Ca}^{40}(3.73 \mathrm{MeV})$ to $100 \%$. Although detailed shell model calculations have not been performed it seems intuitively clear that the particle hole model would not predict such a large decrease in the strength of the lowest $3^{-}$state, particularly between $\mathrm{Ca}^{40}$ and $\mathrm{Ca}^{42}$, because the octupole state is composed of many shell model states, ${ }^{1}$ so that the partial blocking of the $f_{7} / 2$ shell should not cause too large an effect. The same statement is not true of the $5^{-}$state. ${ }^{1}$ We plan to publish this data as soon as possible. (E. P. Lippincott and A. Bernstein)

1. Vincent Gillet and E. A. Sanderšon, Nucl. Phys. 54, 472 (1964). 


\section{Cyclotron Group}

Table 7.I

$3^{-}$and $5^{-}$States in $\mathrm{Ca}^{40,42,44,48}$ and $\mathrm{Ti}^{50}$

$3^{-}$States

\begin{tabular}{|c|c|c|c|}
\hline Nucleus & Energy & $\begin{array}{l}\text { Cross section }\left(32^{\circ}\right) \\
\text { relative to lowest } \\
3^{-} \text {state } \\
\end{array}$ & $\begin{array}{l}\text { Cross section }\left(32^{\circ}\right) \\
\text { relative to } \mathrm{Ca}^{40} \\
3.73 \mathrm{MeV} \text { state } \\
\end{array}$ \\
\hline $\mathrm{Ca}{ }^{10}$ & $\begin{array}{l}3.73 \\
6.29 \\
6.56\end{array}$ & $\begin{array}{r}100 \% \\
24 \% \\
16 \%\end{array}$ & $100 \%$ \\
\hline $\mathrm{Ca}^{42}$ & $\begin{array}{l}3.44 \\
4.70 \\
4.98 \\
5.52 \\
5.68 \\
6.17\end{array}$ & $\begin{array}{c}100 \% \\
32 \% \\
12 \% \\
7.2 \% \\
10 \% \\
15 \%\end{array}$ & $47 \%$ \\
\hline $\mathrm{Ca}^{44}$ & $\begin{array}{c}3.30 \\
4.38 \\
4.90 \\
5.22 \\
5.65 \\
(7.02)\end{array}$ & $\begin{array}{r}100 \% \\
37 \% \\
18 \% \\
15 \% \\
20 \% \\
10 \%\end{array}$ & $34 \%$ \\
\hline $\mathrm{Ca}^{48}$ & $\begin{array}{l}4.50 \\
5.37 \\
7.72\end{array}$ & $\begin{array}{r}100 \% \\
16 \% \\
30 \%\end{array}$ & $32 \%$ \\
\hline $\mathrm{Ti}^{50}$ & $\begin{array}{l}4.42 \\
7.10\end{array}$ & $\begin{array}{r}100 \% \\
38 \%\end{array}$ & $23 \%$ \\
\hline
\end{tabular}

$5^{-}$States

Nucleus

Energy

Cross section relative

$\mathrm{Ca}^{\mathrm{s}}$

4.48

$\mathrm{Ca}^{42}$

4.10

$100 \%$

$\mathrm{Ca}^{44}$

3.91

$55 \%$

4.55

$12 \%$

$\mathrm{Ca}^{48}$

5.73

$22 \%$

$\mathrm{Ti}^{50}$

None found 
Cyclotron Group

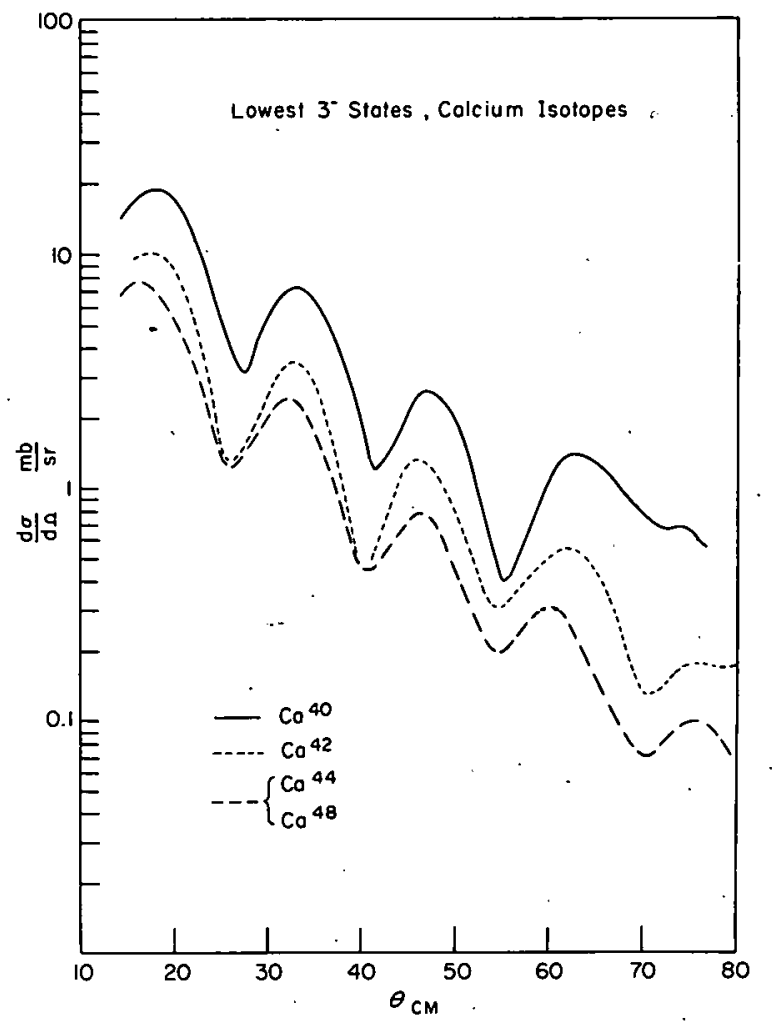

Figure 7.5

Differential cross sections to the lowest $3^{-}$states of $\mathrm{Ca}^{40}, \mathrm{Ca}^{42}$, and $\mathrm{Ca}^{48}$. The curves are lines drawn through the data.

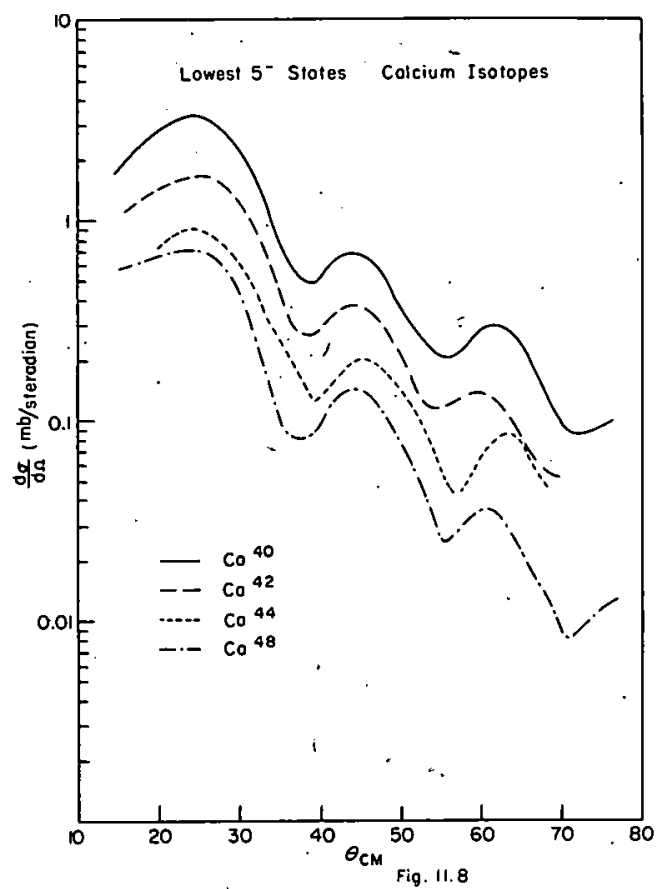

Figure 7.6

Differential cross sections to the $5^{-}$states in $\mathrm{Ca}^{40}, \mathrm{Ca}^{42}$, $\mathrm{Ca}^{44}$ and $\mathrm{Ca}^{48}$. The curves are lines drawn through the data. 
B. $\left(\alpha, \alpha^{\prime}\right)$ on Other Elements

We have taken data_on $\mathrm{Ti}^{46,48}$, and 50 and are presently engaged in data analysis. Also we have done an improved experiment on $\mathrm{Zr}^{90}$. In the interest of brevity these results will not be presented.

The tail of the elastically scattered $\alpha$ particle beam has been reduced from $\approx 10^{-3}$ to $\approx 10^{-4}$. This will enable us to do better inelastic scattering experiments for high $\mathrm{Z}$ elcmente and at small angles for all elements. This has been utilized in the $\mathrm{Zr}^{90}$ experiment and in a $\mathrm{Mo}^{92}$ experiment which is not yet analyzed. (E. Martens, G. Sample, M. Shork, E. Lippincott, J. Alster, and A. Bernstein)

IV. The $(\alpha, p)$ Reaction

A. $\mathrm{Ca}^{42,44}$ and 48 and $\mathrm{Ti}^{48}$ Targets

The investigation of the $(\alpha, p)$ reaction on $f_{7} / 2$ shell nuclei was continued. The experimental arrangement was similar to that used previously for the $\mathrm{Ca}^{40}(\alpha, \mathrm{p}) \mathrm{Sc}^{43}$ data. The total proton resolution was about $150 \mathrm{keV}$. Spectra were taken every 5 degrees from 20 to about 90 degrees and angular distributions were extracted using the P DP -1 data analysis program.

The observed ground state angular distributions are compared in Fig. 7.7. These are all 0 to $7 / 2$-transitions and are quite similar in shape. The solid lines are DWBA fits based on a theory which assumes a three particle stripping reaction to configurations purely in the $\mathrm{f}_{\sqrt{ }} / 2$ shell. ${ }^{1}$ For the case $\mathrm{Ca}^{48}(\alpha, \mathrm{p}) \mathrm{Sc}^{51}$ the. solid line represents the DWBA fit for an assumed $\mathrm{Sc}^{51}$ ground state of $\pi\left(\mathrm{f}_{\sqrt{ }} / 2\right) \nu\left(\mathrm{f}_{5} / 2\right)^{2}$ while the dashed curve assumes a $\mathrm{Sc}^{51}$ ground state of $\pi\left(\mathrm{f}_{7} /{ }_{2}\right) \nu\left(\mathrm{p}_{3} /{ }_{2}\right)^{2}$. As an example of our results we present data in Figs. 7.8 and 7.9 for $\mathrm{Sc}^{47}$ and $\mathrm{V}^{51}$. These spectra are plotted so as to compare the energy levels observed with previous experiments and the theoretical calculations of McCullen, Bayman, and Zamick (MBZ). ${ }^{1}$ For both of these nuclei this theory predicts that only the ground state of the $\mathrm{f}_{7} / 2$ shell contiguration will be seell. This is esoontially what is observed. The paucity of low lying excitations in the $(\alpha, p)$ reaction ends with the onset of p state excitations. As is indicated on the graphs these states occur at $2.42 \mathrm{MeV}$ in $\mathrm{V}^{51}$ and at $0.80,2.91$, and $3.32 \mathrm{MeV}$ in $\mathrm{Sc}^{47}$. Another striking feature is that none of the $\mathrm{d}_{3} / 2$ hole states in this region has been appreciably excited.

The data analysis is presently being completed and a publication of this work will be prepared.

1. B. Bayman and E. Rost, private communication; Bayman, McCullen, and Zamick, Phys. Rev. 134, B 515 (1964). 


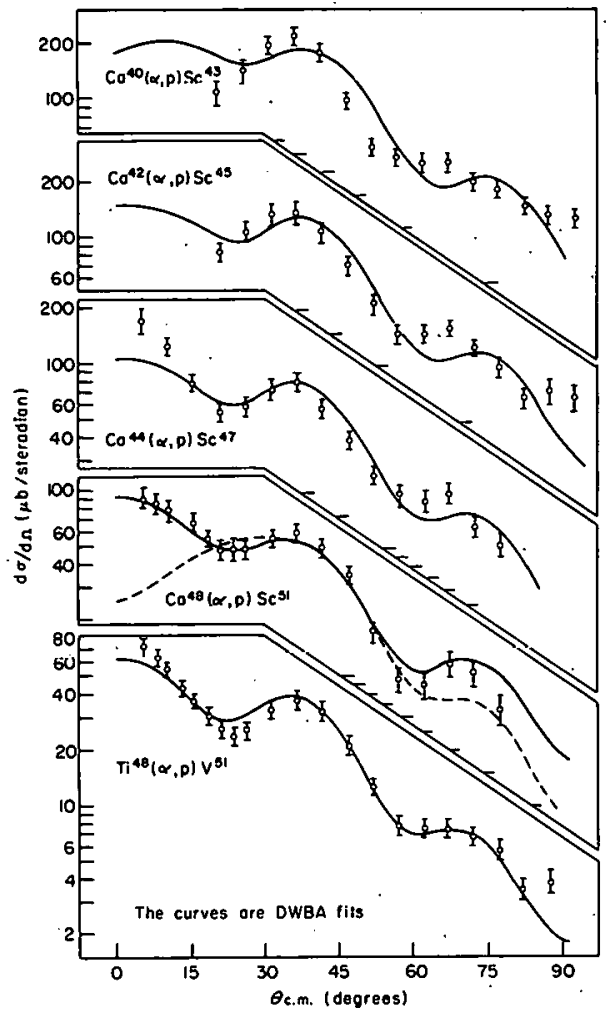

Figure 7.7

Differential cross sections for $(\alpha, \mathrm{p})$ reactions leading to the ground states of the target nuclei. These are all $0 \rightarrow 7 / 2^{-}$transitions. The curves are DWBA fits (see text for details).

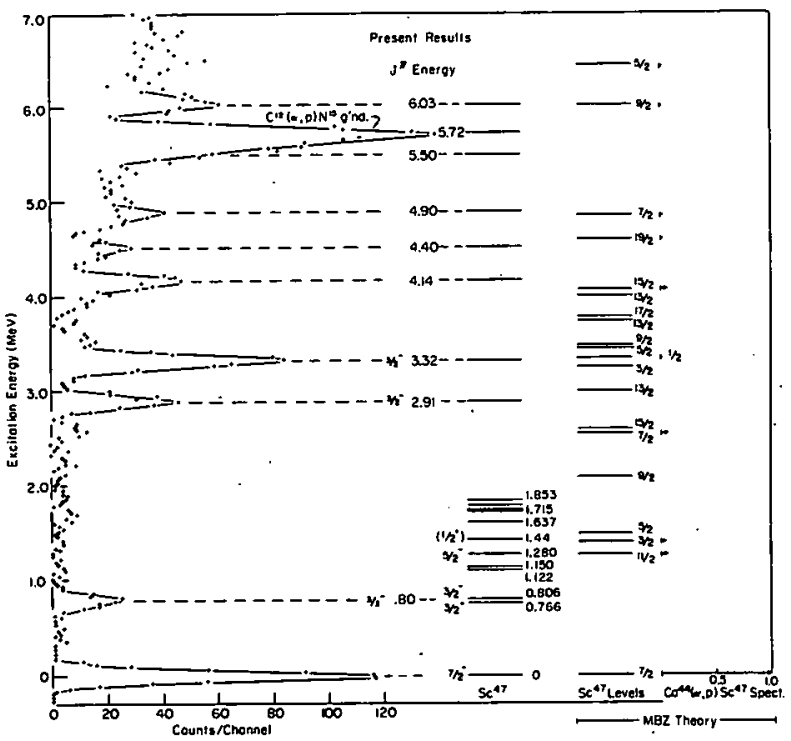

Figure 7.8

Spectrum of the $\mathrm{Ca}^{44}(\alpha, \mathrm{p}) \mathrm{Sc}^{47}$ reaction at the levels marked $\mathrm{Sc}^{47}$ are from previous experiments. The "MB Z Theory" indicates calculated energy levels and spectroscopic factors. (See Ref. previous page) The size of the spectroscopic factors are indicated by the arrows. 


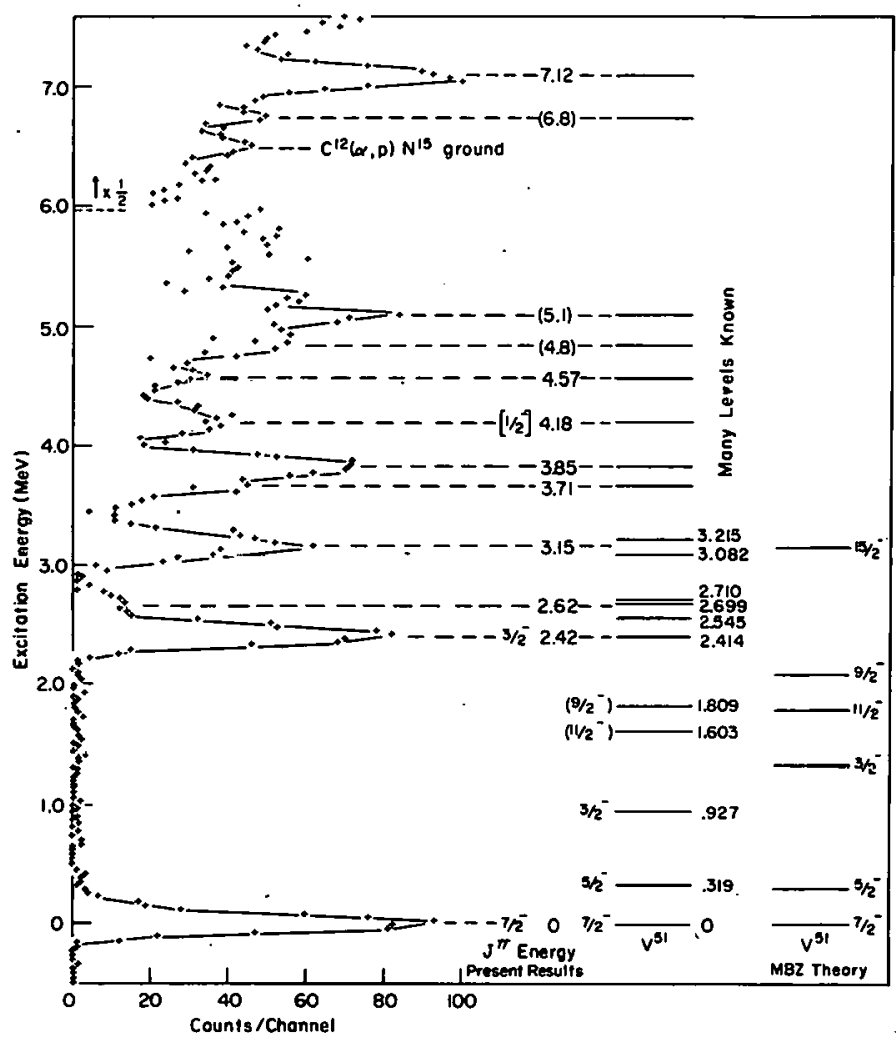

Figure 7.9

Spectrum of the $\operatorname{Ti}^{48}(\alpha, p) V^{51}$ reaction. (See Fig. 7.8 caption for details.)

B. $\mathrm{Fe}^{54}(\alpha, \mathrm{p}) \mathrm{Co}^{57}$

A separate $\left(97.2\right.$ percent) $\mathrm{Fe}^{54}$ target, approximately $1 \mathrm{mg} / \mathrm{cm}^{2}$ thick was used. P roton spectrum was recorded every 5 degrees from a lab angle of 20 to 50 degrees. Angular distributions could not be extracted from the data due to inadequate statistics and resolution. A spectrum is shown in Figure 7.10 with excitation energies for those experimental peaks that could be consistently identified in spectra obtained over an angular region of $20^{\circ}$. The stronis level at $4.60^{\circ} \mathrm{MeV}$ appears to be a doublet with a spacing of less than $60 \mathrm{keV}$. The positions of the lowest levels agree with the states found by August, et al., ${ }^{1}$ which are also indicated in Fig. 7.10.

The $\mathrm{Fe}^{54}$ and $\mathrm{Ca}^{48}$ targets are examples in which the proton may be placed either in the $\mathrm{f}_{7} / 2$ or the next higher shell while the neutrons must be placed in a higher shell. These results illustrate, at least qualitatively, the strong selection rules operating, which differentiate the type of nuclear state that is excited. (R. Ginaven, S. M. Smith, and A. Bernstein)

1. L. S. August, C. R. Cosset, and P. A. Treado, Phys. Rev. 142, 664 (1966). 


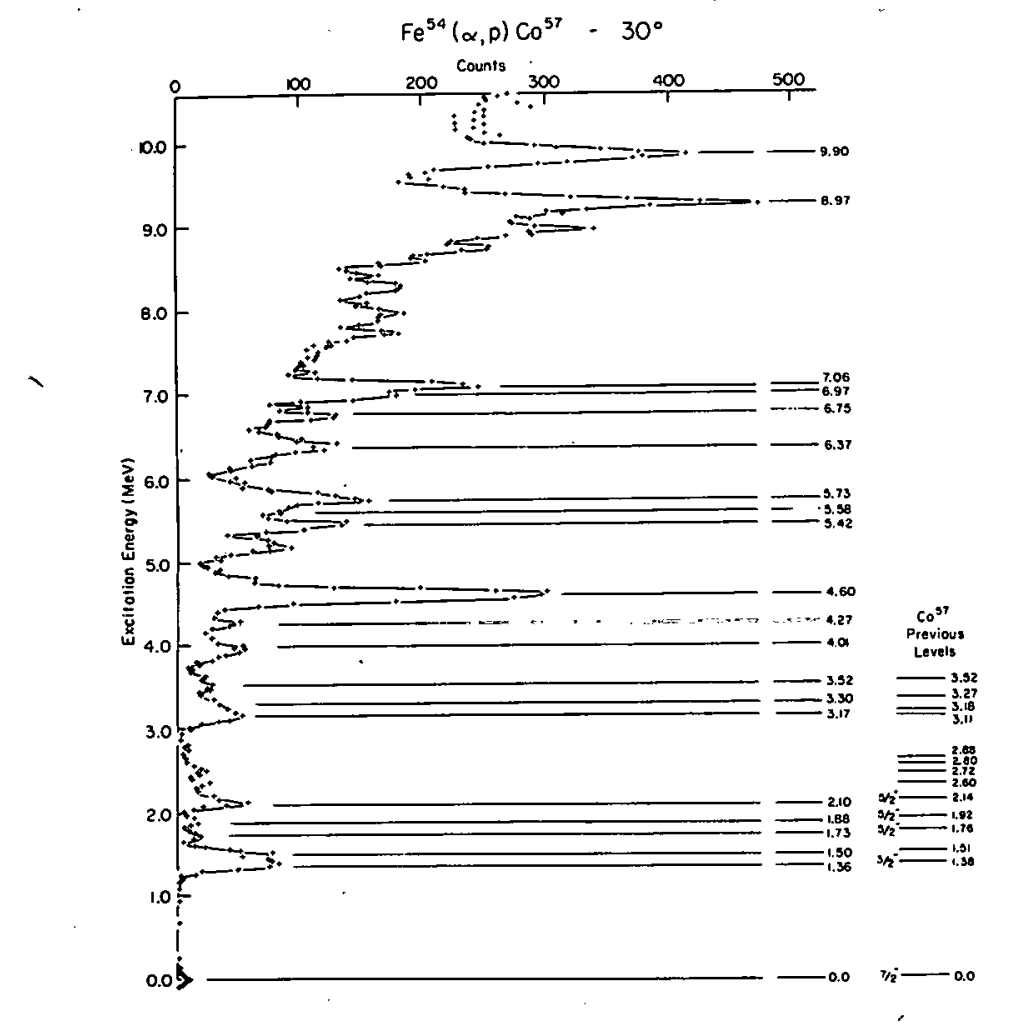

Figure 7.10

A proton spectrum of the $\mathrm{Fe}^{54}(\alpha, \mathrm{p}) \mathrm{Co}^{57}$ reaction at $30^{\circ}$. lab angle. The energies in the left-hand column have been observed in this experiment while those in the right-hand column are from August, et al.

V. $\mathrm{Ca}^{40}\left(\alpha, \alpha^{\prime} \gamma\right)$

We have looked at gamma rays in coincidence with $\alpha$ groups leading to several excited states in $\mathrm{Ca}^{40}$. Figure 7.11 shows the experimental arrangement. The scattering chamber has a flat side so that a gamma detector can be brought close to the target. However, for this experiment the NaI crystal was $5^{\prime \prime}$ removed from the target. The particle detector is inside the chamber and has an aperture approximately $2^{\circ} \times 8^{\circ}$. For all the data presented here on $\mathrm{Ca}^{40}$ the particle detector was at about $30^{\circ}$ from the beam. This corresponds to maxima for $1^{-}$and $3^{-}$ states.

Figures $7.12,7.13$, and 7.14 show the sum over all angles for gamma rays in coincidence with the $6.29,6.56$, and 6.94 groups. The chance gamma rays have already been subtracted out. A smooth line has been drawn through the points. The highest peak position in Fig. 7.12 corresponds to very nearly $3.73 \mathrm{MeV}$ and the shape for such a gamma ray is continued as shown. The resultant difference corresponds to a gamma ray of energy near 1.81, which would correspond to the 6.29 to 4.48 transition. In Fig. 7.14 , the $6.94 \mathrm{MeV}$ state, one can clearly see the direct ground state transition. Together with this we see a branching gamma ray whose full energy peak is very 


\section{Cyclotron Group}

nearly $3.90 \mathrm{MeV}$. Thus we see very roughly equal intensities the $6.94 \mathrm{MeV}$ to 0 and the 6.94 to $3.902^{+}$to 0 branches. The angular correlation measurement was on the 6.94 to ground state - transition, summing all counts of energy between $5 \mathrm{MeV}$ and $7.5 \mathrm{MeV}$.

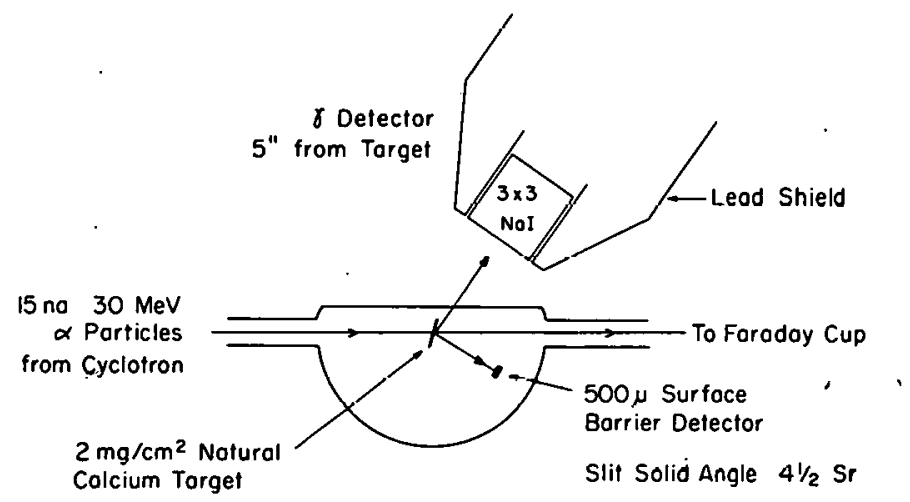

Figure 7.11

Experimental arrangement for the $\left(\alpha, \alpha^{\prime} \gamma\right)$ experiment.

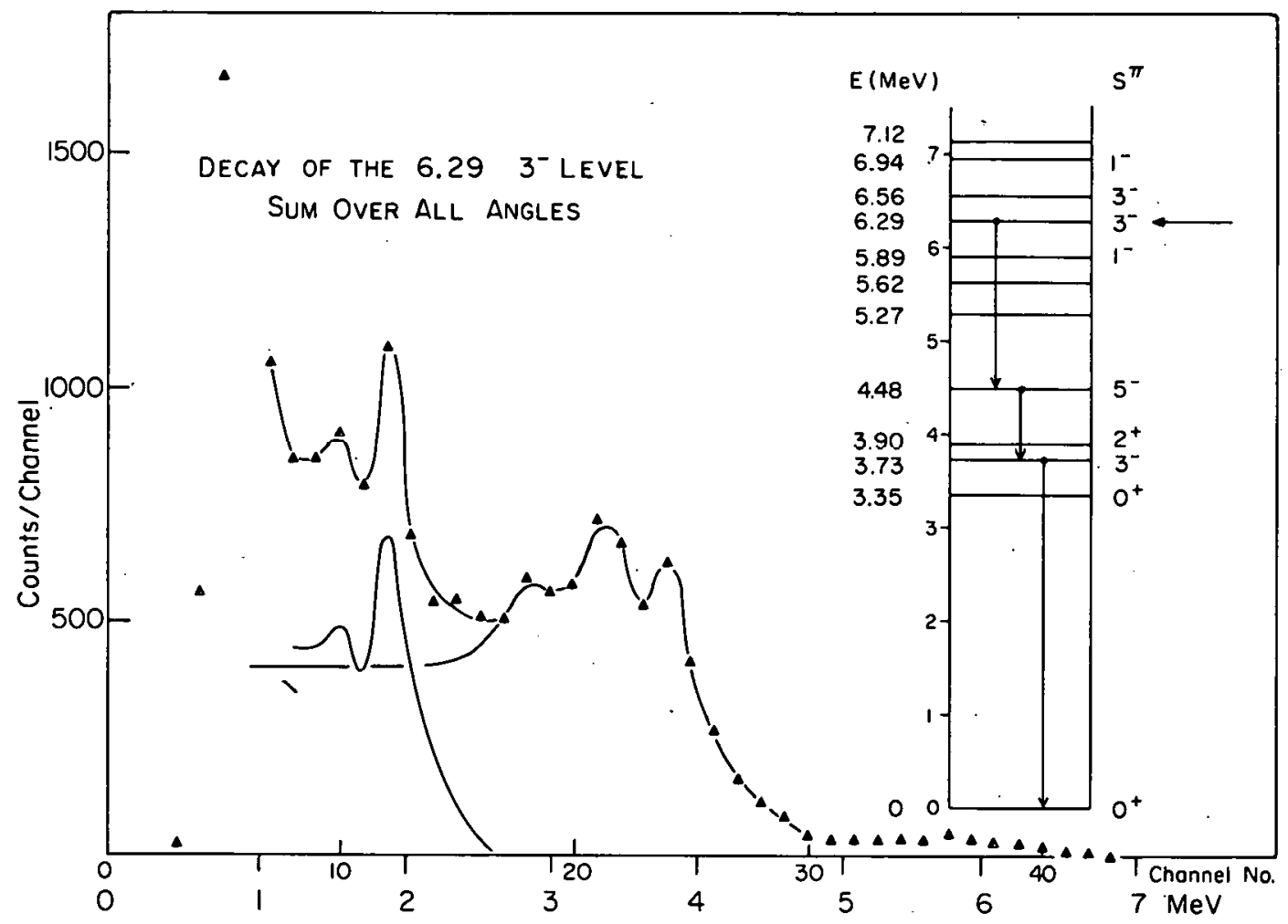

Figure 7.12 


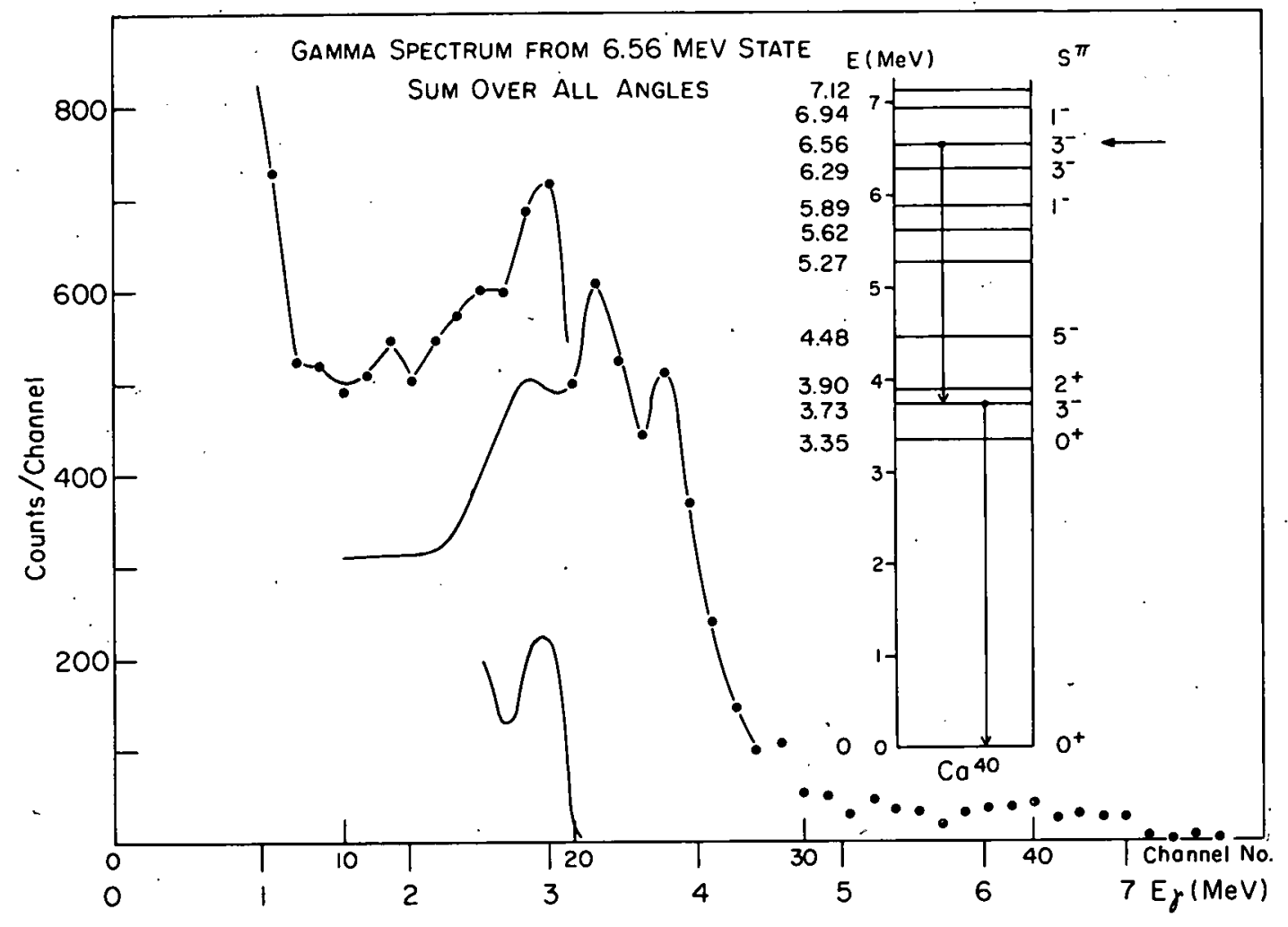

Figure 7.13

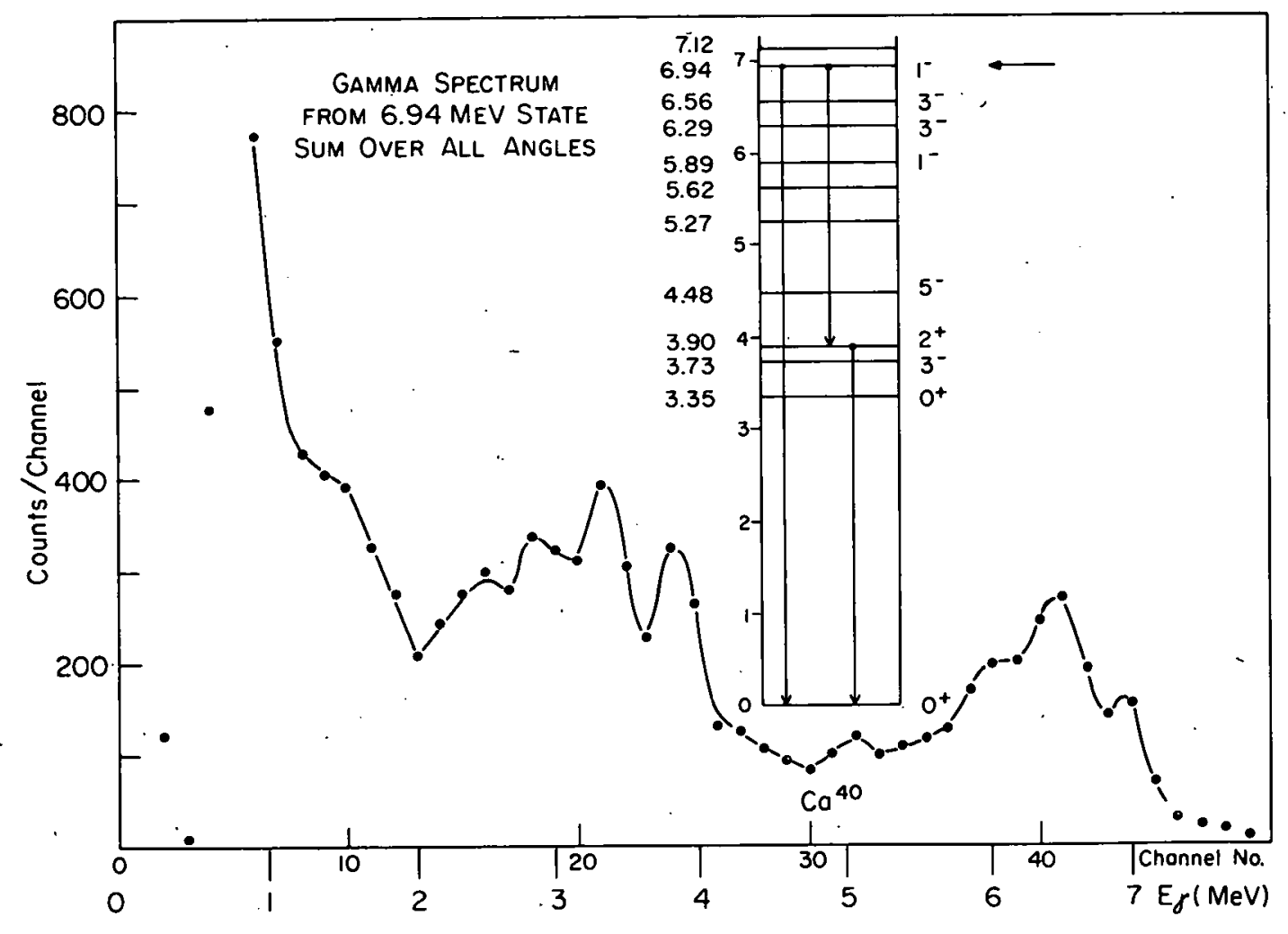

Figure 7.14 


\section{Cyclotron Group}

Figure 7.15 shows the angular correlation observed. Also plotted on the graph are the least squares fitted correlation patterns appropriate to spin 1 and 2 for the $6.94 \mathrm{MeV}$ state. When one considers the data at the angular extremes it is clear that spin 2 is ruled out while spin 1 fits. A spin of 3 or greater presumably would be more complicated, that is more wiggles, and the multipolarity inhibition is formidable relative to E1 even though E1 transitions are isotopically forbidden for this state. Therefore, we think it fair to conclude that at least id predominant part of the group at $6.94 \mathrm{MeV}$, which is excited by alpha particles inelastically scattered at $30^{\circ}$, has spin 1. (K. Nagatani, M. Slade and W. J. Kossler)

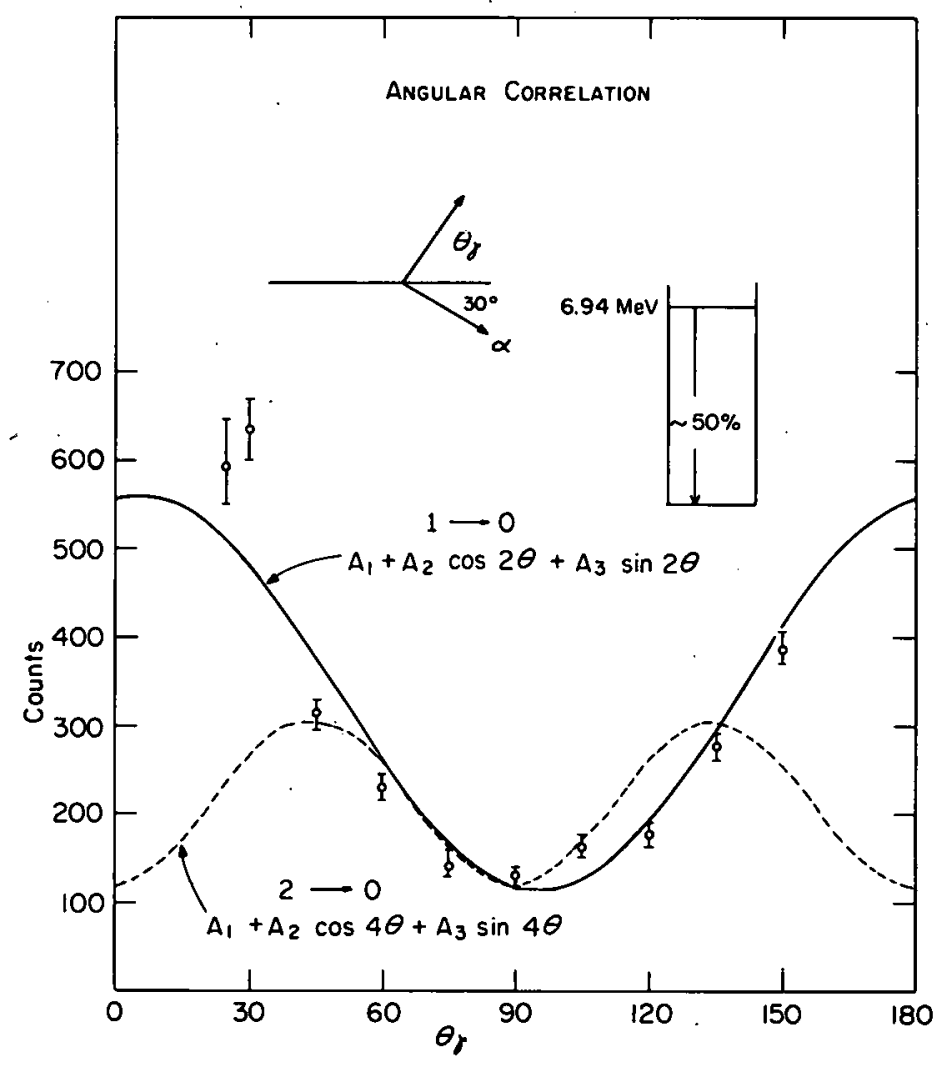

Figure 7.15

Angular correlation of the ground state decay branch of the $\mathrm{Ca}^{40}, 6.94 \mathrm{MeV}$ state.

\section{P roton Pickup Reactions at $26.5 \mathrm{MeV}$}

During the past summer we did an experiment at the University of Colorado Cyclotron in collaboration with $M$. Rickey. We studied $(p, d),(p, t),(p, \alpha)$ and elaștic proton. scattering from $\mathrm{Ca}^{42}$ and $\mathrm{Ca}^{44}$. In Fig. 7:16 we present data from the $\mathrm{Ca}^{42}(\mathrm{p}, \mathrm{d}) \mathrm{Ca}^{41}$ reaction. The most immediate results of the experiment are that the 2.017 and $2.68 \mathrm{MeV}$ states contain the major components of the $d_{3} / 2+s_{1} / 2$ hole states. We can see from the relatively weak excitation of the 2.471 
and $1.949 \mathrm{MeV} 3 / 2^{-}$states that there is relatively little $\left(\mathrm{p}_{3} / 2\right)^{2}$ component in the $\mathrm{Ca}^{42}$ ground state. We also observe three $T=3 / 2$ states at excitation energies of $5.85\left(3 / 2^{+}\right), 6.82\left(1 / 2^{+}\right)$, and 7.13 $\left(7 / 2^{-}\right) \mathrm{MeV}$.

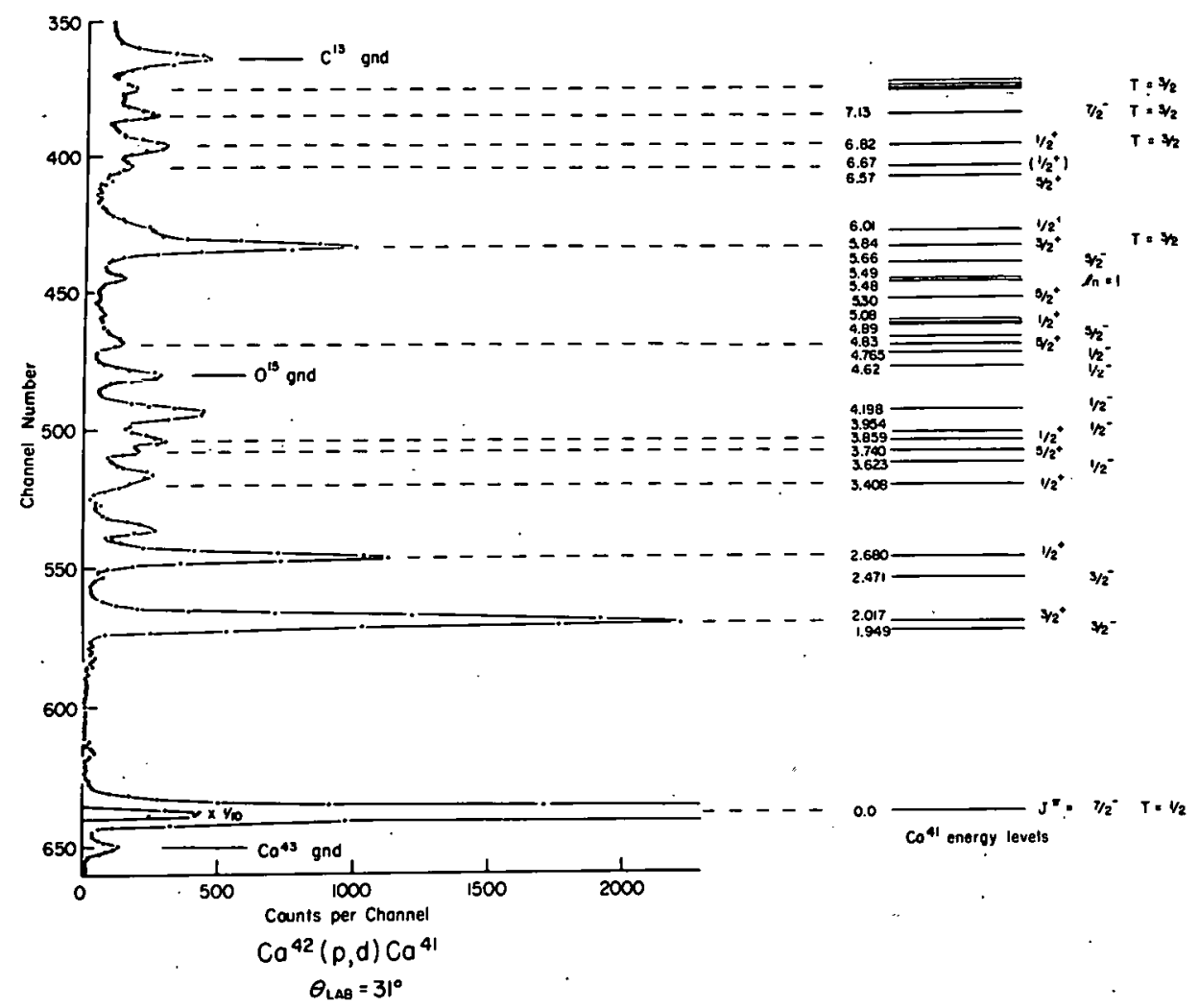

Figure 7.16

Spectrum of the $\mathrm{Ca}^{42}(\mathrm{p}, \mathrm{d}) \mathrm{Ca}^{41}$ reaction at $31^{\circ}$. The levels of $\mathrm{Ca}^{41}$ have been obtained from Ref. 1 .

In Fig. 7.17 we present a spectrum from the $\mathrm{Ca}^{44}(\mathrm{p}, \mathrm{d}) \mathrm{Ca}^{43}$ reaction. Again one can see the $\mathrm{d}_{3} / 2$ hole state at $0.992 \mathrm{MeV}$ and the $s_{1} / 2$ hole state at $1.959 \mathrm{MeV}$. The lowest $\mathrm{T}=5 / 2$ analogue state appears at $7.97 \mathrm{MeV}$. We note that a second $3 / 2^{+}$state at $1.395 \mathrm{MeV}$ is weakly excited compared to the lower $3 / 2^{+}$state. We also note the peaks for the $3 / 2^{-}$states at $0.594 \mathrm{MeV}$ and the $5 / 2^{-}$state at $9.373 \mathrm{MeV}$. These states would be forbidden if they were pure $\left(f_{7} / 2\right)^{3}$ configura tions and the ground state of $\mathrm{Ca}^{44}$ was a pure $\left(\mathrm{f}_{7} / 2\right)^{4}$ configuration.

For the sake of brevity we shall not present results of the other reactions until the analysis is complete. At the present time relative differential cross sections have been obtained for most of the data. We anticipate completion of the analysis in the near future. (S. M. Smith and A. Bernstein)

1. T. A. Belote, A. Sperduto, and W. W. Buechner, Phys. Rev. 139, B 80 (1965). 


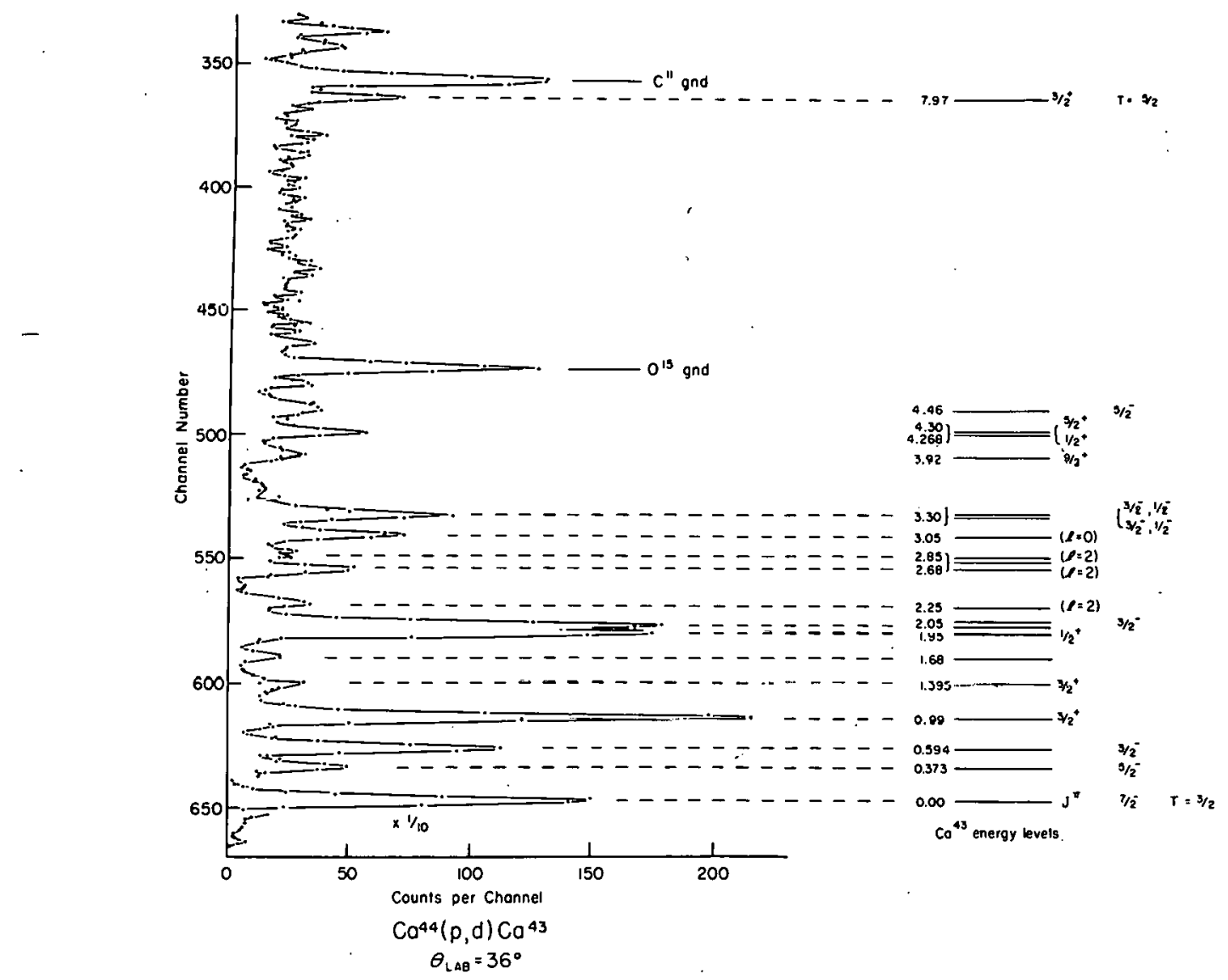

Figure 7.17

Spectrum of the $\mathrm{Ca}^{44}(\mathrm{p}, \mathrm{d}) \mathrm{Ca}^{43}$ reaction at $36^{\circ}$. The levels of $\mathrm{Ca}^{43}$ have been taken from Ref. 1 .

\section{Analysis of Data}

The P DP - 1 data-analysis program, discussed in the previous progress report, has been modified so that it reads data stored on magnetic tape. This improves the data analysis by allowing us to analyze conveniently a small energy interval as the scattering angle is varied. Fits to the data are stored on magnetic tape and subsequently plotted by a 1401 computer.

The PDP - 1 computer has also been used to convert paper tape to magnetic tape'for use in data analysis programs for the 7044 computer. This has been accomplished for one-dimensional data, which has been chiefly applied to an $\left(\alpha, \alpha^{*}\right)$ data, and two-dimensional data for the $(\alpha, 2 \alpha)$ experiment.

A new program called MANIB has been written which takes the output data from CYCLOPS (Cyclotron data analysis program) or the P DP -1 data analysis program and assigns $Q$ values. This process takes kinematics and ionization losses in the target into account. This program also does laboratory to center -of-mass conversion and assembles the data in a useful way. (T. P rovost, R. Ginaven,...W. J. Kossler, and S. M. Smith)

1. W. E. Dorenbusch, T. A. Belote, and Ole Hansen, to be published. 
VIII. DWBA Analysis

During the spring semester a program was initiated to calculate form factors for inelastic scattering, following previous work done in this laboratory by N. S. Wall. Our approach differs in that we use the $\mathbf{7 0 4 4}$ computer to generate the form factors. At the time of this writing some of the programs have been written and debugged. With the advice of M. Veneroni, of the Theoretical Group, the form factors using quasi-particle wave functions have been generated for the $\mathrm{Ni}$ isotopes.

Form factors have also been calculated for the $2^{+}$state of $\mathrm{Ti}^{50}$. We have been able to fit the data about as well as the usual collective model does.

We have written a DWBA code for the 7044 computer called TANYA. This code is based on optical model wave functions calculated in ABACUS, but with an improved integration method. This program has been tested by making internal checks and by comparing it with results obtained by the Oak Ridge code JULIE. At present TANYA is mainly geared towards $\left(\alpha, \alpha^{\prime}\right)$ reactions with a variety of form factors options. We intend to use this flexibility in fitting our data.

(T. Provost, W. J. Kossler, and A. Bernstein)

\section{P UB LICATIONS}

R. W. Bauer, A. M. Bernstein, G. Heymann, E. P. Lippincott, and N: W. Wall, " $\mathrm{Ca}^{40}\left(\alpha, \alpha^{\prime}\right)$ : A Test of Particle-Hole Calculations", Phys. Letters 14, 2 (1965).

R. W. Bauer, G. Heymann, W. Kossler, N. S. Wall, and C. R. Gruhn,, "Study of the $\mathrm{Ca}^{\mathbf{4} 0}(\alpha, 2 \alpha)$ Reaction;" Rev. Mod. Phys. 37, 369 (1965).

A. Bernstein and T. P rovost, "Nuclear Reaction Data Analysis with a Small Computer", Bull. Am. Phys. Soc. 10, 478 (1965).

A. Bernstein and E. J. Martens," The $\mathrm{Zr}^{90}\left(\alpha, \alpha^{\prime}\right)$ Reaction at $30.5 \mathrm{MeV}, "$ Bull. Am. Phys. Soc. 10,122 (1965).

E. P. Lippincott and A. Bernstein, "Elastic and Inelastic Scattering of $30.5 \mathrm{MeV}$ Alpha Particles on $\mathrm{Ti}^{50 "}$, Bull. Am. Phys. Soc. 10, 122 (1965).

\section{THESES}

E. J. Martens, "Elastic. and Inelastic Scattering of Alpha Particles by Zr ${ }^{90 ",}$ M.S. (Feb. 1965). G. Sample, "The $\mathrm{Ti}^{48}\left(\alpha, \alpha^{\prime}\right)$ Reaction", B.S. (Dec. 1965). 


\section{THEORETICAL GROUP

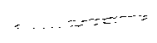

I. Introduction

Since the last progress report much of the anticipated expansion of the Theoretical Group has taken place. As expected, this has produced a lively stimulating atmosphere, which is of obvious benefit to both the regular staff and graduate student body. The latter group has also been able to draw upon the visitors for advice and direction. This year nearly eighty applications for post-doctoral research positions were received. A considerable number of more experienced theoretical physicists from other universities and national laboratories will be spending their sabbaticals or leaves with us in the coming academic year. It is a pleasure to record the return of Prof. V. F. Weisskopf to M.I.T. and the Theoretical Group.

The work done by the Theoretical Group can be conveniently categorized as follows. Some studies are phenomenological in nature, i.e., they attempt to correlate and order data in terms of a model and/or selection rules. On the other hand, there are more fundamental inquiries, which attempt to explain these models and selection rules. In this introduction we shall mention a few of the developments of each type, carried on by the Theoretical Group, which seem to us to be of particular interest.

In particle theory, fundamental theory at present attempts to explain and predict the quan tum numbers of the various resonances of the baryons and bosons. The two principal frameworks in which these problems have been considered are first the unitary symmetry models and second the more dynamic bootstrap mechanism. Huang and Mueller have extended the method developed by Huang and Low for the study of the existence and properties of bootstrap solutions to more complex solutions. In particular, it is shown that no unsubtracted bootstrap solution exists for the Chew-Low theory and for the scattering of an octet of scalar mesons by an octet of baryons in the limit of $S U(6)$ symmetry. These results indicate that the consequences of such a concept are extremely sensitive to the nature of the approximations used if they are valid at all.

A number of papers have been done on the SU(6) symmetry scheme and its $\widetilde{U}(12)$ generalization. These investigations are of the phenomenological type. They attempt to analyze da $\overline{t a}$ on the basis of a symmetry and simple assumptions on the symmetry properties of the interaction terms. Of particular interest are the electro-magnetic mass splittings, which have been calculated in the $\mathrm{SU}(6)$ scheme on the basis of a simple mass-splitting Hamiltonian. The A quantum number, which has been considered to be a boson property, has been applied by one of our distinguished visitors, D. C. P easlee, to weak interactions. He finds that the combination AP (P =parity) is conserved in non-leptonic decays to the order for which the A quantum number selection rule is satisfied. A number of predictions, which can be checked experimentally, have been made.

The relativistic three-body and $\mathrm{N}$-body problem is of central importance in any dynamical consideration of models for particles. Bronzan has been able to produce a problem of this type (charged scalar static theory), which has an exact solution. Federbush has shown that the pro- 
posed extension by Weinberg of the Fadeev equations to more than three particles possesses unwanted homogeneous solutions.

Field theory, particularly quantum_electrodynamics, has been of continued interest here. Developments may yield equations for the bare charge and may verify the Regge hypothesis of Gell-Mann, Low, etc., for nucleon interaction with a heavy vector meson in a conserved vector theory.

In the intermediate energy range, Lomon has continued to investigate phenomenologically nucleon-nucleon, pion-nucleon and kaon-nucleon interactions. For the latter (boson-nucleon) he has demonstrated the great importance of inelastic thresholds at energies below the threshold. An important development in the boundary condition model for nucleon-nucleon scattering is the great improvement that occurs in the understanding of the scattering data when a single $\rho$ meson exchange potential is included in the description of the interaction outside the boundary condition core. The model now includes single and double pion exchange. Single $\rho$, w and $\eta$ exchange with the experimental masses and experimental coupling constants, the $w$ and $\eta$ coupling constants being obtained from the SU(3) symmetry. Excellent agreement is also obtained with photodisintegra tion of and electron scattering by the deuterons.

One of the major problems in nuclear theory is the determination of the properties of finite as well as infinite nuclei directly from the nuclear forces as determined from nucleonnucleon scattering. If the usual hardcore potential or boundary condition potential is used, some form of the Brueckner method must be employed. This method is used by Shakin, Lomon and Sprung, while in the other calculations (Kerman, et al.), the hardcore and boundary condition is replaced by a softcore. It turns out that it is possible to obta in excellent agreement with nucleonnucleon data with such a core. This potential is now being used in the Hartree-Fock calculation of several nuclei. So far, the only complet $\mathrm{H}-\mathrm{F}$ calculations have been made for the ground states of $\mathrm{O}^{16}$ and $\mathrm{Ca}^{40}$ using a separable potential and assuming spherical symmetry. Binding energy per particle was found to be too small; single particle energies were in better agreement with the data, although the spin-orbit splitting is too large. The discrepancy in the binding energy per particle is probably accounted for by second order effects. A number of Hartree-Fock calculations for deformed nuclei have been made. The use of parity-mixed Hartree-Fock states has been under active investigation.

Nuclear reactions continue to be the focus of considerable interest in the Theor etical Group . The concept of doorway states and intermediate resonances has been delineated and is being applied to isobar analogs, to neutron strength functions and to inelastic scattering. The effect of the Pauli principle on nuclear reactions has been spelled out, and then evaluated for the scattering of neutrons by nuclei for a simplified model, and is currently being applied to stuffing. An important advance has been made by Villars and Junkin, who show how a generalized linked-cluster series for an optical potential for a deuteron interacting with a nucleus can be obtained.

Of a number of optical model studies one of particular interest is a new understanding of the maxima in the total cross section of the neutron scattering data. These have been found not to be associated with single particle resonances in a single angular momentum state, but rather in a diffraction type phenomenon with several angular momentum states cooperating. 


\section{Quantum Electrodynamics}

We have shown that the divergent part of photon-self energy, when one omits all the diagrams in perturbation theory that contain insertions of the photon-self energy, as a function of the cut-off, has the form $F\left(\alpha_{0}\right) \log \left(\lambda^{2}\right)$. Here, $F\left(\alpha_{0}\right)$ is a finite power series in the "bare" coupling constant. We have further found a method of calculation which completely avoids the problem of "overlapping" divergences. Using this method we have recalculated the fourth-order result, first obtained by Jost and Lutinger some years ago. However, our method is simple enough so that the corresponding sixth-order calculation is feasible, and at the moment is in pro-gress. The interest in this problem is to investigate the equation $F\left(\alpha_{0}\right)=0$ as a possible equation for the bare coupling constant. We have also a method for going slightly beyond perturbation theory in that if the Bethe-Salpeter kernel for electron positron scattering is represented by by calculating the contribution to the photon-self energy represented by

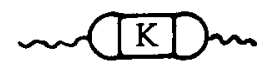

We also determine the sum of all contributions of the form

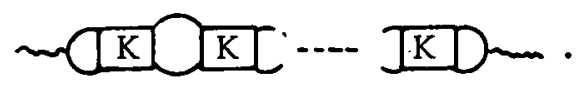

In other words, we get the contribution of the sum of all "ladders" in P. Yock; work in collaboration with Professor M. Baker, University of Washington, Seattle, Washington)

\section{Variational Formulation of Quantum Field Theory}

A variational formulation of field theory has been developed in analogy with some recent work in statistical mechanics of DeDominicis and Martin. A detailed study of the $\phi^{3}$ theory has been made that shows that this theory can be completely characteriżed by a first order functional differential equation for the mass operator or the vertex function (where the renormalized propagator plays the role of the functional variable) and by a non-linear integro-differential equation which determines the propagator itself. Information of different nature can be obtained from such an approach. In particular a study of the weak coupling limit of the equation for the mass operator shows that in the approximation in which the space time continuum is replaced by a finite lattice, the self-energy shows an essential singularity for vanishing values of the coupling constant.

(H. D. Dahmen and G. Jona-Lasinio) 
IV. Infrared Contrïbutions to Intermediate State Sums in Quantum Electrodynamics

A method has been developed for incorporating the Bloch-Norsdsieck approximation into intermediate state sum expansions in quantum electrodynamics, so as to explicitly include the contributions of soft photons to these sums. This accomplishes the removal of infrared divergences usually incurred in approximations involving the cutting off of such intermediate state sums, and in addition, within the framework of such approximations, gives strong high energy convergence properties to the theory. The formalism developed is applied to modify Fainberg's work on quantum electrodynamic dispersion relations. An asymptotic form for the electron's charge form factor is derived, and is shown to be consistent with second order perturbation theory, and a series expansion for the charge renormalization constant $Z_{a}$ is obtained, all of whose terms are finite, except for those which arise from states containing only hard photons and no pairs. In a separate development, a comparison is made of the various methods which have been developed for treating the radiative corrections to scattering cross sections due to soft photons. (R. P errin and E. L. Lomon)

\section{Verification of the Reggeisation Hypothesis in Nth Order}

Work was started on the problem of verifying the Reggeisation hypothesis of Gell-Mann, et al., for the nucleon in a conserved vector current theory of the interaction of a nucleon with a heavy vector meson. A general method for determining the leading behavior at large values of the momentum transfer of planar Feynman graphs with spin was worked out, written up and submitted to the Journal of Mathematical Physics. The method was then applied to the specific problem of determining the leading and lower order terms of the ladder graphs in the nucleon-meson scattering process in addition to certain important renormalized graphs. The results of Cheng and $\mathrm{Wu}$ were obtained, verifying the Reggeisation hypothesis to sixth order. However, the present method allows a ready generalization to higher orders. This work has been written up and submitted to the Journal of Mathematical Physics. Present work concerns verification in the eighth order in the hope that the cancellation mechanism will become so transparent as to yield verification in the nth order. (J. V. Greenman)

\section{Topology and Feynman Diagrams}

Some application of Algebraic Topology to the study of Feynman Diagrams has been investigated. This work led to the following two publications:

1) The Calculation of Some Homology Groups Relevant to Sixth Order Feynman Diagrams.

2) A Note on Non-Landau Singularities. 
' (Both to be soon published in the J. Math. Phys.) As a product of earlier investigations, the following paper will soon appear in the Annals of Physics: An Example of the Mandelstam Representation in P erturbation Theory. (P. Federbush)

VII. Bound on the Coupling Constant Derived from Dispersion Theory

An upper bound on the coupling constant appearing in relativistic dispersion relation has been obtained under some assumptions on the number of zeroes of the left hand discontinuity. (H. D. Dahmen and A. S. Reiner)

\section{Exact Bootstrap Solutions to' the Low Equation}

The work of Huang and Low on exact bootstrap solution of the Low equation (J. Math. Phys. $6,795,1965)$ has been extended in two ways:

(a) A new technique is found that enables one to discuss the existence and properties of bootstrap solutions without having to solve the Low equation explicitly.

(b) The result that no unsubtracted bootstrap solution exists is extended to include all $2 \times 2$ crossing matrices and to some $4 \times 4$ crossing matrices. The last case includes the Chew Low theory. This result is also obtained for the scattering of an octet of scalar mesons with an octet of baryons, in the limit of SU(3) symmetry. (K. Huang and A. H. Mueller)

\section{A Model for Strong Interactions}

A start was made on what promises to be a large project: representation of boson and excited baryon states in terms of an explicit baryon-antibaryon model. The model is realistic in the sense of having no quarks, for which the ordinary rules of exclusion principle, parity conservation, and orbital motion, are often abrogated; the elementary particles are the "bare" baryon octet. Although crude, the model provides immediate physical insight and should be capable of further refinement. One paper on boson beta decay has been written embodying the simplest of these notions; a more complicated problem of baryon magnetic moments has arisen out of discussions with Professor H. Feshbach. (D. C. Peaslee)

\section{Three Body States in Particle Physics}

We have been concerned with the effect of three particle states in static field theories.. An extended version of the Lee model was devised, which has a non-trivial vertex function, two and 


\section{Theoretical Group}

three particle intermediate states, and the possibility of a bound state. ${ }^{1}$ Techniques developed in this work were applied to the more complicated charged scalar theory. Numerical work was done on the relative importance of crossing and three particle unitarity for low energy dynamics. ${ }^{2}$ An exact two meson solution of the charged scalar static theory was later developed. ${ }^{3}$ (J. A. Bronzan)

\section{Multiparticle Potential Scattering}

An attempt is in progress to generalize the Fadeev equations to systems of more than three particles. By limiting oneself to Yukawa type potentials it is hoped to take advantage of analyticity. The Weinberg equations, a possible starting point, have been shown to have the fault of possessing unwanted homogeneous solutions. ( $P$. Federbush)

\section{High Energy Scattering from Deuterons}

Work has been completed on an investigation of the relationship between the energy dependence of the Glauber shadow effect in high energy scattering from deuterons and $\ell$-plane analyticity considerations. It is found that persistence of the shadow effect at high energies may indicate that elastic diffraction scattering is dominated by a fixed $\ell$-plane singularity. This work was performed with E. Abers (UCLA), H. Burkhardt (University of Birmingham), and C. Wilkin (BNL). (V. L. Teplitz)

\section{Ladder Approximation in Field Theory}

A modified ladder approximation scheme in relativistic field theory has been investigated, and its consequences for the simplest possible matrix elements of the product of two field operators have been studied in detail. Results concerning the two-point Wightman function are reported in MIT-2098-151. (G. Roepstorff)

1. J. B. Bronzan, Phys. Rev. 139, B751 (1965).

2. J. B. Bronzan and R. W. B rown, Annals of Physics (to be published).

3. J. B. Bronzan, submitted to J. Math. Phys. 


\section{Poincare Group}

A decomposition of representations of the Poincare group into representations of the homogeneous subgroup has been found and the weight function for the subrepresentations has been determined. This group theoretic method has been used to give an alternative proof for the YostLehmann-Dyson representation. Possible extensions to representations of the B ergmann-Weyl type for the three- and four-point Wightman function have been considered. (G. Roepstorff)

\section{Meson-Baryon Interaction with'B roken SU(3) Symmetry}

A.Ph.D. thesis has been completed treating meson-baryon scattering with broken SU(3) symmetry by means of a many-channel Low equation. 'The main topics discussed are the mass formula with threshold effects taken into account, and the possibility of a 27 -fold resonance. It is found that despite threshold effects, the Gell-Mann - Okubo formula remains valid, seemingly by accident. It is found that a 27 -fold resonance should not exist. (A. H. Mueller)

\section{Higher Symmetries}

We are considering the possibility of unconventional $(8,8)$ assignments for the baryons in the $U(3) \times U(3)$ symmetry scheme, as well as the possibility of $S U(3)$ at $p_{z}=\infty$ being an exact classification. (F. E. Low and T. Yao)

\section{SU(6) Symmetry}

The electromagnetic mass splittings of the baryon multiplet were studied in the SU(6) symmetry scheme. In the calculation we assume the electromagnetic mass-splitting Hamiltonian to be

$$
\mathrm{H}_{\mathrm{em}}=\mathrm{aQQ}+\overrightarrow{\mathrm{bM}} \cdot \overrightarrow{\mathrm{M}}
$$

where $Q$ transforms like the electric-charge operator, and $M$ transforms like the magnetic moment operator. Seven mass relations are obtained for the baryons ( 56 representation)

$$
\begin{aligned}
& \Xi^{-}-\Xi^{0}=\left(\Sigma^{-}-\Sigma^{+}\right)-(\mathrm{n}-\mathrm{p}) \\
& \mathrm{N}^{* 0}-\mathrm{N}^{*+}=\mathrm{Y}_{1}^{* 0}-\mathrm{Y}_{1}^{*+}=\mathrm{n}-\mathrm{p} \\
& \mathrm{N}^{*-}-\mathrm{N}^{* 0}=\mathrm{Y}_{1}{ }^{*-}-\mathrm{Y}_{1}^{* 0}=\Xi^{*-}=\Xi^{* 0}=(\mathrm{n}-\mathrm{p})+\left(\Sigma^{-}+\Sigma^{+}-2 \Sigma^{0}\right) \\
& \mathrm{N}^{*^{-}}-\mathrm{N}^{*++}=3(\mathrm{n}-\mathrm{p}) .
\end{aligned}
$$

The comparison with experi mental results wherever available is very well satisfied. This work has been published. (T. Yao) 
XVIII. Coupling Constants in B roken U(12) Symmetry

The baryon -meson vertex has been investigated in the framework of the relativistic SU(6) scheme $\widetilde{U}(12)$. The effects of symmetry breaking were studied using the spurion technique. For an SU(3) breaking spurion which is a member of the self adjoint representation 143 of $\widetilde{U}(12)$, all 132 baryon meson coupling constants can be expressed in terms of only three coupling parameters. Many relations hold between the coupling constants. In particular the sum rules obtained for the observed decuplet width are in reasonable agreement with experiment. (D. Flamm)

XIX. Weak Interactions and the A-Parity Quantum Number

The application of the charge parity quantum number $A$ to very weak interactions has been studied. It appears that non-leptonic decays conserve AP, when $P$ is real parity. A similar rule is postulated for the hadron currents in leptonic decays. Observed relations between $\Lambda \rightarrow \pi p$ and $\Xi^{-} \rightarrow \Lambda \pi$ verify the AP rule with a deviation of order 15 percent from A-symmetry in the baryon octet. Simple relations are predicted between $\Xi \rightarrow(\Lambda, \Sigma)$ and $(\Lambda, \Sigma) \rightarrow N$ leptonic decays. The structure of non-leptonic baryon decays is almost completely specified by introduction of one further symmetry postulate, which does not fit readily into the SU(3) framework. The AP rule sheds no light on $\mathrm{K}_{2}^{0} \rightarrow 2 \pi$ decay. (D. C. Peaslee)

\section{$\mathrm{XX}$. Inelastic P rocesses and Levinson's Theorem}

The possibility of diagonalizing the $\mathrm{S}$ matrix when inelastic processes are present is studied in order to generalize Levinson's theorem. The preliminary studies were made on a simple soluble model by Blankenbecler. (M. Cassandro)

\section{Weinstein-Caldwell Effect}

This effect gives rise to the production of false peaks in mass plots due to final state mixing effects. We have so far considered only a simple non-relativistic model. We do not yet know whether or not $\omega$ and $\rho$ production on $\mathrm{H}$ can show this effect, but we expect to find out soon. (F. E. Low and G. Jona-Lasinio) 
XXII. Photo $-\mathrm{P}$ roduction and Electron $-\mathrm{P}$ roduction of $\rho^{0}$

Data of the Bubble Chamber Group on $\gamma+p \rightarrow p+\rho$ have been analyzed in terms of the vacuum Regge trajectory. The data can be fitted with the choice $\alpha(\mathrm{t})=1+\mathrm{kt}, \mathrm{k} \cong 1.1(\mathrm{BeV})^{-2}$. Work is in progress to use this information to predict $e+p \rightarrow e+p+\rho$. In particular, it is hoped that the electromagnetic production $\mathrm{e}+\mathrm{p} \rightarrow \mathrm{e}+\gamma+\mathrm{p}$ can be kinematically separated from - Lip the strong production, thus enabling one to measure directly the coupling constant for $\gamma \rightarrow \rho$. (R. Therus and K. Hilang)

\section{Photoproduction of Vector Mesons}

One and two pion exchange contributions to the photoproduction of $\omega$ and $\rho^{0}$ mesons at a few GeV/c were calculated. (P. C. M. Yock)

\section{3 -Body Forces in Nuclei}

We have estimated the effect of the longest range part of the nuclear 3 -body forces in the triton and in 3-particle configurations of larger nuclei. The contribution of this force to the binding of the triton is about 100 kilovolts and the splitting of the 3 -particle configuration in Ca ${ }^{43}$ is about 10 kilovolts. (H. K. Quang and A. K. Kerman)

\section{Boundary Condition Model for Nucleon-Nucleon Scattering}

The inclusion of the one $\rho$ meson exchange potential has made an important change in the character of our fit to the $n-p$ data. With this term added to the OP EP and TP EP potentials we now obtain excellent agreement with both the $\mathrm{p}-\mathrm{p}$ and $\mathrm{n}-\mathrm{p}$ scattering data with (1) a $\pi$ meson -nucleon coupling constant that is common to both the OP EP and TP EP potential and in excellent agreement with the value of this constant as determined from meson-nucleon scattering, (2) experimental $\pi$ meson masses, (3) experimental $\rho$-meson mass, and (4) the experimental $\rho$-meson nucleon coupling constant as determined from the nucleon electromagnetic form factors.

We have also considered the effect of adding the potential generated by the single $w$ and $\eta$ exchange. These additional terms yield a slight improvement in our fit of the data. The experimental $\mathrm{w}$ and $\eta$ masses are used; the coupling constants determined empirically come close to that given by $\mathrm{SU}(3)$ from the $\rho$ and $\pi$ coupling constants. (E. Lomon and H. Feshbach) 


\section{The Bethe-Salpeter Equation}

The B ethe-Salpeter equation for the case of a scalar meson exchange has been integrated numerically for positive kinetic energies. Substantial agreement is obtained with the results of Schwartz and Zemach which was published at the time thi s work was concluded. (R. Saenger)

\section{B oundary Condition Model and the B ethe-Salpeter Equation}

A relativistic generalization of the boundary condition model using the B ethe-Salpeter equation is developed, the boundary condition being applled at least initially at $R$ (the four distance) equal to zew. The appropriate form of the boundary condition at this point has been determined. (R. P. Zia and H. Feshbach)

XXVIII. The Analytic Continuation of an $\mathrm{S}_{11} \mathrm{P}$ ion-Nucleon Amplitude to the $\mathrm{P}_{11}$ Amplitude

The McDowell Symmetry is used to obtain the unique $\mathrm{P}_{11}$ pion-nucleon amplitude related to the simple analytic form of the $S_{11}$ amplitude derived previously from a two channel boundary condition model. The a posteriori justification for the extension of the too simple $\mathrm{S}_{11}$ amplitude over several $\mathrm{BeV}$ is the resulting satisfaction of the stringent $\mathrm{P}$ state threshold requirement, and a qualitative agreement of the $\mathrm{P}$ state amplitude with experimental information. (H. Goldberg and E. L. Lomon)

XXIX. Kaon-Nucleon Scattering and Threshold $\mathrm{K}$ P roduction

Using the boundary condition model, it is shown that the rise of the I=0 S state KN phase shift near $P_{L}$ (kaon laboratory momentum) $=800 \mathrm{MeV} / \mathrm{c}$ can be attributed to the effect of the $\mathrm{K}^{*} \mathrm{~N}$ threshold. Approximately $2.8 \mathrm{mb}$ of $\mathrm{K}^{*}$ production at $1140 \mathrm{MeV} / \mathrm{c}$ is predicted for that channel. If the interchannel coupling is reduced for the $\mathrm{I}=1$ channel in the ratio given by isovector exchange, the experimental production cross-section is obtained and the earlier fit to the $\mathrm{I}=1 \mathrm{~S}$ state phase shift altered negligibly. Elastic boundary condition fits are given the $P$ and $D$ waves. The boundary radius, consistent with theory is $0: 45 \mu^{-1}$ except for one D state. The $0.7 \mu^{-1}$ radius required in the latter case indicates a significant repulsive potential tail. (W. J. deBonte and E. L. Lomon) 
The reactions induced by an energetic $\mathrm{K}$ beam such as

$$
\mathrm{K}^{-}+(\mathrm{N}, \mathrm{Z}) \rightarrow \pi^{-}+(\mathrm{N}-1, \mathrm{Z}) \Lambda
$$

can be used to determine the energy levels of the hypernucleus $(N-1, Z){ }_{\Lambda}$ by studying the spectrum of the $\pi^{-}$. When the energy of the hypernucleus is large enough it becomes unstable against neutron emission and not unstable against $\Lambda$ emission. Resonances can occur in this energy range. The question of the existence of an SU(3) symmetry analog level similar to the isobar analogue is discussed. The symmetry breaking force analogous to the Coulomb force in the isobar analogue case is shown to be the one pion exchange potential. (H. Feshbach and A. Kerman)

XXXI. Photoproduction of Pions from Nuclei

A study of photopion production from nuclei has been undertaken in view of the imminence of the high energy electron linac. The main emphasis will be to determine what can be learned about nuclei. (A. K. Kerman and L. Saunders)

XXXII. Nucleon-Nucleon Potentials

The search is continuing for soft core nucleon-nucleon potentials, which fit the two-body data and are useful.in Hartree-Fock calculations for the finite nucleus problem. In addition, we have begun investigating the possibility of replacing our finite core potentials with separable nonlocal potentials inside the core. (W. Bassichis, C. Bressel, B. Levy and A. K. Kerman)

XXXIII。P roperties of Nuclear Matter by Perturbation Theory with Finite Two-Body Potential

The problem of applying perturbation theory to a finite nucleon-nucleon potential has been greatly simplified. Explicit formulae have been derived for the first and second order contributions from all terms of the mos $t$ general two nucleon potential consistent with invariance principles and with explicit momentum and angular momentum dependence up to quadratic terms. The nine dimensional integrations of the second order terms have been reduced to double quadratures, by the evaluation of the seven "kinematic" integrations -- all those that can be done without specify ing the interaction. The results of these integrations define a set of generalized Euler functions $G_{n}(q, x)$, which play the role for the exchange terms that the function $p(u)$ of Euler plays for the 
direct terms. These functions have been evaluated numerically, once and for all, for $q=0(.1) 7.0$ (in units of the Fermi momentum) and $x=q(0.1) q+2.0$, and $n=0,2,4$, which is the range required for physical applications. $G(q, x)$ is symmetric in $(q, x)$ and vanishes for $q$ or $x$ negative or for $|x-q|>2.0$. A Fortran program ha's been developed which computes the perturbation theory contributions for a given potential, given a table of the required Bessel transforms of the radial potential shapes. A related program computes this table for a class of local potential models. Applications have been made to the finite potential determined by $\mathrm{C}$. Bressel (thesis, MIT, 1965) which provides a precise fit to two-body scattering data. First results indicate that this potential is still too strong to justify application of perturbation theory.

Work in progress consists of:

(i) Formulation of requirements on a finite potential which will allow application of perturba tion theory;

(ii) Plans to calculate the binding energy of nuclear matter according to Brueckner's theory, using the same potential and incorporating three body cluster effects. (D. Sprung)

XXXIV. Properties of Nuclear Matter according to the Boundary Condition Model of Nuclear Forces

These calculations, described in the November 1964 report, are still in progress.

(D. Sprung)

XXXV. Quantized Vortex Rings in Superfluid Helium

A phenomenological theory is devised to explain some recent experiments of Careri, et al., which seem to indicate the existence of quantized vortex rings in superfluid helium. The theory successfully $y_{f}$ explains all the details of the experiments, and will be published in the Aug. 30, 1965, issue of the Physical Review. (K. Huang and A. C. Olinto)

XXXVI. Nucleon-Nucleon Boundary Condition Methods for Nuclear Matter

It is shown that, as a consequence of the energy independence of the boundary condition $f$ on the radial Schroedinger wave function $u$ at $r_{0}, u=0$ for $r<r_{0}$, and that the boundary condition is equivalent to replacing $\mathrm{Vu}$, in the equation for $\mathrm{u}$ in $\mathrm{r}<\mathrm{r}_{0}$, by an expression linear in $u\left(r_{0}+\epsilon\right)$ and $u^{\prime}\left(r_{0}+\epsilon\right)$. The Bethe-Goldstone wave function $u^{N}$ is determined by the same replacement in its integral equation. $B$ ut $u^{N} \neq 0$ in $r<r_{0}$, so that $u^{N}$ depends on an extra parameter $b$, the boundary condition at $r_{0}-\epsilon$. P seudo-potentials at $r_{0}$ which give the boundary condition exactly are presented. The self-consistent single particle potential energy in nuclear matter is investigated with a 
simple S state boundaxy condition, without a potential tail. The effective mass approximation is found inadequate. For the same interaction, the effect of summing the hole-hole contributions is found to be important for the two-body correlations, and small but significant for the saturation and binding energy. A calculation is made with a more realistic model with a potential tail. A modified Moszkowski-Scott type expansion, separated at $r_{0}$, is used. Convergence is poor and binding is not obtained. More recent nucleon-nucleon boundary condition models may give convergence and binding. (M. M. Hoenig and E. L. Lomon)

\section{Hartree-Fock Calculations in Nuclei}

An application of the Hartree-Fock (HF) method to calculation of the structure of finite nuclei is presented. The non-local, separable potential of Tabakin is used as the two-body interaction. The calculation is carried out by writing the HF equations in an oscillator basis and applying the Moshinsky transformation to relative coordinates. The closed-shell nuclei $\mathrm{O}^{16}$ and $\mathrm{Ca}^{\Delta 0}$ are considered. Under the assumption that they are spherical, their binding energy per particle is found to be -2.41 and $-3.47 \mathrm{MeV}$ for $\mathrm{O}^{16}$ and $\mathrm{Ca}^{40}$, respectively. This discrepancy is made up in large part by the second order potential energy. The single-particle energies show better agreement with data, but have too much spin-orbit splitting; namely, $10.2 \mathrm{MeV}$ for $1 \mathrm{p}$ states in $\mathrm{O}^{16}$ and 11.13 and $14.59 \mathrm{MeV}$, respectively for the $1 \mathrm{p}$ and $1 \mathrm{~d}$ states in $\mathrm{Ca}^{40}$. The $\mathrm{rms}$ radii for $\mathrm{O}^{16}$ and $\mathrm{Ca}^{40}$ were found to be 2.38 and 2.96 fermi, compared to experimental values of $2.64 \mathrm{f}$ and $3.52 \mathrm{f}$, respectively. Corrections for Coulomb force and center-of-mass motion have also been calculated. (A. K. Kerman, J. P. Svenne, and F. M. H. Villars)

\section{Hartree-Fock Calculations in Nuclei}

Work is continuing on a large program of Hartree-Fock calculations in finite nuclei using realistic 2 -particle forces with soft or non-local cores. We have found that the purely non-local potential of Tabakin gives some binding in oxygen and $\mathrm{Ca}$ and about the right radius, and that the first soft core potential of Bressel, Kerman, and Lomon is still too strong for a Hartree-Fock theory. However, mixtures of these seem to be promising. This work is being done in collaboration with A.M. Lockett of the Los Alamos Scientific Laboratory. ' (J. P. Svenne, A. K. Kerman, and F. Villars) 
XXXIX. Hartree-Fock Calculations for Finite Nuclei

We have available a computer program capable of calculating the unirestricted HartreeFock approximation for nuclei up to $A=90$. Currently work is under way using the method of Lagrange multipliers to study the dependence of the energy surface on deformation, and on the lifting of parity and time reversal restrictions in the single particle wave functions. (W. Bassichis, J. P. Svenne, and A. K. Kerman)

\section{Hartree-Fock Calculations for $\mathrm{Zr}^{90}$}

We have investigated the convergence of Hartree-Fock calculation for the nucleus $\mathrm{Zr}^{90}$. Our aim was to find how many oscillator functions are necessary to describe the single particle wave functions adequately for calculating the energy of a heavier nucleus than those previously investigated. We find that three oscillator functions are quite adequate to obtain convergence of the total energy.

It is also interesting to note that shell model calculations of the low lying spectrum of $\mathrm{Zr}^{90}$ with the same force and wave functions give a spectrum similar to the experimental one. But second order effects are undoubtedly important and these will be investigated. (A. K. Kerman, J. P. Svenne, and Y. Waghmare)

\section{Hartree-Fock Calculations in Nuclei}

A program has been written to do Hartree-Fock calculations for non-axially symmetric nuclei. The energy surface as a function of $\beta$ and $\gamma$, the deformation parameters are being investigated for $\mathrm{C}^{12}$ and $\mathrm{Mg}^{24}$. The program enables one to do a systematic investigation starting from spherical shapes, and then going over to axially symmetric deformation, and finally to deformations of a completely general type including the effects of parity mixing. (M. K. Pal and A. Stamp)

XLII. Hartree-Fock Ground State and Residual Pairing Interactions

The corrections to the HF ground state due to residual pairing interactions were solved assuming a model. The model assumes constant strength of pairing, and degenerate two-hole two-particle excited configurations. Specific calculations have also been done without these assumptions. This work has been communicated to the Washington meeting of the American Physical Society, April 1966. (M. K. Pal) 
XLIII. Correlated Basis Functions in the Theory of Finite Nuclei

A unitary model -operator approach has been developed for nuclear structure problems. By the use of a cluster-expansion technique we are able to construct an effective hamiltonian specified in the basis provided by harmonic-oscillator functions. ${ }^{1}$ The requirement of the convergence of the cluster expansion leads to a generalized separation method for the treatment of singular (i.e., hard-core) nucleon-nucleon interactions. ${ }^{3}$

Once thé effective hamiltonian has been determined it is possible to apply this theory to most low-energy nuclear physics problems. We are interested in problems such as binding energies, ${ }^{4}$ transition rates, ${ }^{2}$ spin-orbit splittings, ${ }^{4}$ nuclear spectra ${ }^{5}$ and nucleon-nucleus scattering. (C. Shakin).

XLIV. Matrix Elements for Effective Hamiltonian Method

P rograms have been written to determine the effective-interaction matrix elements for the Yale and Hamada-Johnston potentials. ${ }^{3}$ Tables of these matrix elements are being constructed: (C. Shakin, M. H. Hull, and Y. R. Waghmare)

XLV. Effects of the Tensor Force in Effective Hamiltonian Method

It is found that the tensor force admixes very high-energy orbitals into the nuclear wave function. The renormalization of the nucleon-nucleon force due to this effect is treated in second-order perturbation theory. It is clear that this is a very important feature of the effective interaction. The second-order terms have been calculated for various size parameters of the oscillator wave functions. (C. Shakin and M. Tomaselli)

XLVI. Hartree-Fock Calculations with Effective Hamiltonian

This is probably the most significant aspect of our work. Once we have specified the effective hamiltonian it is possible to apply the Hartree-Fock method by expanding the unknown

1. J. DaP rovidencia and C. M. Shakin, Ann.Phys. (NY) 30, 95 (1964).

2. J. DaP rovidencia and C. M. Shakin, Nucl. Phys. $65, \overline{54}$ (1965); Nucl. Phys. 65, 75 (1965).

3. M. H. Hull, Jr., and C. M. Shakin, Phys. Letters 19, 506 (1966).

4. C. M. Shákin and Y. R. Waghmare, Phys. Rev. Letters 16, 403 (1966)

5. C. Shakin, J. Svenne, and Y. R. Waghmare, "Shell-Mode Studies with Realistic Potentials. I" ( to be submitted for publication.) 
orbitals in the harmonic-oscillator basis. The expansion coefficients are then the variational parameters for the Hartree-Fock problem.

This procedure essentially allows for improvements in the "long-range" aspects of the wave function. The part of the wave function describing the situation when the particles are close together is already specified (non-variationally) by the unitary substitution of correlated basis functions.

Hartree-Fock calculations for $\mathrm{O}^{16}$ and $\mathrm{Ca}^{40}$ are under way. Preliminary results for the binding energy of $\mathrm{O}^{16}$ are excellent ( 7.5 MeV per particle). (C. Shakin, J. Svenne and Y. R. Waghmare)

XLVII. Nuclear Spectroscopy using Effective Hamiltonian

Studies of the spectra of $\mathrm{Zr}^{90}$ and $\mathrm{Ti}^{50}$ have been completed and a paper has been prepared describing the results. Agreement with experimental data is good. ${ }^{1}$

Studies of the spectra of $\mathrm{P}^{206}, \mathrm{Bi}^{210}, \mathrm{Sc}^{42}$ and various other nuclei are under way. Also, a study of doublet splittings has been undertaken. We also hope to study problems in pairing theory with our effective interaction. (C. Shaking, Y. R. Waghmare, J. Svenne and M. Toma selli )

XLVIII. Hartree-Fock Calculations in the s-d Shell

Work on the stability of axial Hartree-Fock solutions for odd A and odd-odd nuclei in the first half of the s-d shell are in progress. (S. Tewari)

XLIX. Effective Interaction in Finite Nuclei

Matrix elements suitable for shell model calculations in the 1p shell have been computed applying an extension of the Eden-Emery method to the Hamada-Johnston potential. Tensor forces are handled by solving coupled Bethe-Goldston equations. The effect of the non-hermiticity of the $t$-matrix on the two body matrix elements is under investigation. (A. D. MacKellar and R. L. Becker)

1. C. Shakin, J. Svenne, and Y. R. Waghmare, "Shell Model Studies with Realistic Potentials.I" (to be submitted for publićation). 


\section{Pari ty Mixed Hartree-Fock States}

It has been suggested (notably by $\mathrm{K}$. Bleuler) that due to the tensor force, a Hartree-Fock trial function of definite parity is unstable (that is, gives a saddlepoint of the energy, not a minimum).

This point has been verified by means of a simple device: Let $\phi_{0}$ be a solution (of say even parity) of the HF equation. We then construct the trial function

$$
\phi(\lambda)=e^{i \lambda \Sigma \bar{\sigma}_{i} \cdot r_{i}} \quad \phi_{0}
$$

and determine $\mathrm{E}(\lambda)=.\langle\phi(\lambda)|\mathrm{H}| \phi(\lambda)>$.

Now

$$
E(\lambda)=\left\langle\phi_{0}\left|e^{-i \lambda \Sigma \sigma \cdot r} H e^{i \lambda \Sigma \sigma \cdot r}\right| \phi_{0}\right\rangle=\left\langle\phi_{0}|\widetilde{H}(\lambda)| \phi_{0}\right\rangle
$$

$\tilde{H}(\lambda)$ is easily calculated, and $E(\lambda)-E(0)$ can then be estimated. One finds that instead $E(\lambda)$ has two minimer at $\lambda= \pm \lambda_{0}$, and $\lambda_{0} R \sim 1$ ( $R=$ nuclear radius). The energy gain $E_{0}-E\left( \pm \lambda_{0}\right)$ is estimated to be of the order $\sim 10 \mathrm{~A}^{1 / 3} \mathrm{MeV}$.

In this approximation, one may obtain a degenerate pair of states

$$
\phi_{ \pm}=\left(\phi\left(\lambda_{0}\right) \pm \phi\left(-\lambda_{0}\right)\right.
$$

These two states are practically orthogonal, since the overlap

$$
\left.<\phi\left(\lambda_{0}\right) \phi\left(-\lambda_{0}\right)\right\rangle=\left\langle\phi_{0}\left|e^{-2 \mathrm{i} \lambda_{0} \Sigma \vec{\sigma} \cdot \vec{r}}\right| \phi_{0}\right\rangle
$$

is estimated to be of the order

$$
e^{\left.-2 \lambda\}<(\Sigma \sigma \cdot r)^{2}\right\rangle} \approx e^{-2\left(\lambda_{0} R\right)^{2} A^{2 / 3}}
$$

A more realistic description allows the system to oscillate the "parity well" $E(\lambda)$; that is, one uses wave function

$$
\phi=\int d \lambda g(\lambda) \phi(\lambda)
$$

The equation for $\mathrm{g}$ is in the process of being solved. (F. Villars)

\section{Theory of Nuclear Rotation.}

Nuclear rotational energies are conventionally calculated by the semi-classical method of Inglis or Thouless and Valatin. These methods deal only with wave functions of indeterminate angular momentum, and give the energy as a function of angular velocity $\vec{\omega}$. A new method has been developed which projects the Hamiltonian of the system on the subspace of all states of a definite angular momentum J. A general prescription is given to construct this the projected Hamiltonian $H^{(J)}$. It has the form

$$
\mathrm{H}^{\mathrm{J}}=\mathrm{H}^{(0)}+\sum_{\mathrm{A}=\mathrm{i}}^{3} \mathrm{H}_{\mathrm{A}}^{(1)} \mathrm{J}_{\mathrm{A}}+\frac{1}{2} \sum_{\mathrm{A}, \mathrm{B}}^{3} \mathrm{H}_{\mathrm{AB}}^{(2)} \mathrm{J}_{\mathrm{A}} \mathrm{J}_{\mathrm{B}}+
$$


$\mathrm{J}_{\mathrm{A}}$ are body-fixed angular momentum components,

$$
\mathrm{J}_{\mathrm{A}}=\left(\vec{J} \cdot \overrightarrow{e_{A}}\right) \text {, }
$$

$\vec{e}_{A}$ being the unit vectors defining the body fixed coordinate system: $H^{(0)}, H^{(1)}, H^{(2)}$ are operators commuting with both $\vec{J}$ and $\overrightarrow{e_{A}}$.

The eigenvalues problem for $\mathrm{H}^{\mathrm{J}}$ leads to diagonalizing the operator

$$
<\mathrm{JK}\left|\mathrm{H}^{\mathrm{J}}\left(\mathrm{x}_{\mathrm{i}}, \mathrm{P}_{\mathrm{i}}\right)\right| \mathrm{JK}^{\prime}>=\mathrm{H}^{(0)}\left(\mathrm{x}_{\mathrm{i}} \mathrm{P}_{\mathrm{i}}\right) \delta \mathrm{KK}^{\prime}+\sum_{\mathrm{A}} \mathrm{H}_{\mathrm{A}}^{(1)}\left(\mathrm{x}_{\mathrm{i}^{\prime}}, \mathrm{p}_{\mathrm{i}}\right)\left(\mathrm{JK}\left|\mathrm{J}_{\mathrm{A}}\right| J K^{\prime}\right)+\ldots
$$

This may be done approximately by using a Hartree-Fock solution for the eignevalue problem of $\mathrm{H}^{(0)},\left(\mathrm{x}_{\mathrm{i}}, \mathrm{p}_{\mathrm{i}}\right)$, and a perturbation technique for the residual terms of (2).

To test the nature of results so obtained, a solution based on these views has been worked out for a two-dimensional case. It is found that the expression for the rotational moment of inertia is essentially identical to the formula of Thouless and Valatin.

A manuscript on the 3-dimensional case is being prepared. (F. Villars)

LII. Formulae for Tensor Operators in the Peierls-Yoccoz Theory

In the P eierls-Yoccoz approach to rotational states, deformed Hartree-Fock wave functions $\phi_{\mathrm{K}}\left(\mathrm{K}=\right.$ eigenvalue of $\left.\mathrm{J}_{3}\right)$ are projected on a fixed value of $\mathrm{J}$ and $\mathrm{J}_{3}=\mathrm{M}$, to give wave functions of $\psi_{\mathrm{KJM}}$

$$
\psi_{\mathrm{K}, \mathrm{JM}}=\int \frac{\mathrm{d} \Omega}{8 \pi^{2}} \quad \mathrm{D}_{\mathrm{KM}}^{+\mathrm{J}}(\Omega) \mathrm{R}(\Omega) \phi(\mathrm{x})
$$

$D^{J}$ being a Wigner-D-function, and $R$ a rotation operator. It is then of interest to reduce matrixelements $<\psi_{\mathrm{K}^{\prime}, \mathrm{J}^{\prime} \mathrm{M}^{\prime}}\left|\mathrm{T}_{\mathrm{K \mu}}\right| \psi_{\mathrm{KJM}}>$ to integrals over the original Hartree-Fock wave functions $\phi_{\mathrm{K}}(\mathrm{x})$. The general reduction formula is

$$
\begin{aligned}
<\psi_{K^{\prime}, J^{\prime} M^{\prime}}\left|T_{K \mu}\right| \psi_{K J M}>= & \frac{<J^{\prime} M^{\prime} \mid K \mu, J M>}{2\left(2 J^{\prime}+1\right)} \sum_{\lambda}\left\langle J^{\prime} K^{\prime}\right| K \lambda, J\left(K^{\prime}-\lambda\right)>\int_{-1}^{+1} \mathrm{~d} \cos \beta d_{K^{\prime}-\lambda, K}^{J}(\beta) \\
& <\phi_{K^{\prime}}\left|T_{K \lambda} e^{-i \beta J y}\right| \phi_{K^{\prime}}>
\end{aligned} .
$$

General methods for handling the $\beta$-integration are described. A series of illustrative examples have been worked out: Scalars (normalization of $\psi$, energy), vectors (magnetic moment operator) and the electric quadrupole tensor. The origin of $\mathrm{K}$-selection rules is displayed.

In the case of the energy, a technique to avoid the small angle expansion for $d^{J}(p)\left(\alpha_{K K}^{J}(\beta)\right)=$ $1-\frac{\beta^{2}}{4}\left(J(J+1)-K^{2}\right)$ is presented, which give $E_{J}$ as the ratio of two power series in $J(J+1)$. (G. Cooper and F. Villars) 


\section{Doorway States and Intermediate Resonances}

The extension of reaction formalism to include the effects of doorway states has been com pleted. The averaging process has been carried through and an intermediate complex potential model derived. The resulting intermediate structure in strength functions, elastic and inelastic cross sections has been made explicit. The empirical situation has been reviewed and a number of analyses of data for intermediate structure are in progress. The part of this investigation concerned with a nucleus reaction formalism including the effects of doorway states is now being written up for publication. (H. Feshbach, A. K. Kerman, and R. H. Lemmer)

\section{Isobaric Analogue States}

Work is continuing on a theory of isobaric analogue states, using methods of scattering theory developed for a discussion of intermediate structure in nuclear cross sections. We have computed the internal damping of the analogue states for $\mathrm{Zr}$ and $\mathrm{Sr}$ and found it to be very small, the damping being less than a few kilovolts. This work is being carried on in collaboration with S. Fallieros and R. Venter of the Bartol Research Foundation. (A. F. R. de Toledo Pisa and A. K. Kerman)

LV. Coupled Channel Studies of the Analog Resonances Corresponding to Single Particle States

We are using a coupled channel program to study the nature of the first three analog states in the reaction ${ }^{*} \mathrm{Sr}^{88}+\mathrm{p}$. It is expected that the width and shapes of these resonances can be completely explained using parameters which are fixed by elastic scattering off the resnnanre and p-n reaction at higher energies. The mechanism for the resonance is the coupling of the incident proton channel to the closed channel consisting of a neutron plus the analog of the target.

(E. Auerbach, C. Dover, A. K. Kerman, and R. H. Lemmer)

\section{Solution of Coupled Differential Equations}

Work has been completed on a computer program for the solution of a system of coupled radial Schrydinger equations

$$
\left[\frac{d^{2}}{d r^{2}}+k_{\alpha}^{2}\left(1-\frac{v_{\alpha}}{E}\right)\right] \quad u_{\alpha}(r)=\sum_{\beta} v_{\alpha \beta}(r) U_{\beta}(r)
$$

\footnotetext{
* Experiments have been performed in this laboratory by Cosman, Enge and Sperduto.
} 
where the $\mathrm{V}_{\alpha \beta}$ are local coupling terms. This will form the nucleus of a larger program in which the option of removing the locality restriction will be available.

The coupling terms to be considered are (1) those arising from a potential appropriate to vibrational and rotational excitations

$$
\mathrm{V}(\overrightarrow{\mathrm{r}}, \alpha)=\mathrm{V}(\mathrm{r})+\mathrm{r} \frac{\mathrm{dV}(\mathrm{r})}{\mathrm{dr}} \sum_{\ell, \mathrm{m}} \alpha_{\ell, \mathrm{m}} \mathrm{Y}_{\ell \mathrm{m}}+\frac{1}{2} \mathrm{r}^{2} \frac{\mathrm{d}^{2} \mathrm{~V}(\mathrm{r})}{\mathrm{dr} \mathrm{r}^{2}} \sum_{\substack{\ell, \mathrm{m} \\ \ell, \mathrm{m}}} \alpha_{\ell \mathrm{m}^{\prime}} \alpha_{\ell \mathrm{m}^{\mathrm{Y}} \ell^{\prime} \mathrm{m}^{\prime} \mathrm{Y}_{\ell}{ }^{\prime} \mathrm{m}^{\prime}}
$$

i.e., a form which allows for the excitation of one- and two-phonon target states and (2) those arising from nucleon-nucleon potential of various standard forms of radial dependence

$$
\begin{aligned}
& \text { (a) } \mathrm{V}_{0} \delta\left(\overrightarrow{\mathrm{r}} \cdot \overrightarrow{\mathrm{r}^{\prime}}\right) \\
& \text { (b) } \mathrm{V}_{0} \mathrm{e}-\beta\left|\overrightarrow{\mathrm{r}}-\overrightarrow{\mathrm{r}^{\prime}}\right|^{2} \\
& \text { (c) } \mathrm{V}_{0} \frac{\mathrm{e}-\beta\left|\overrightarrow{\mathrm{r}}-\overrightarrow{\mathrm{r}^{\prime}}\right|}{\beta\left|\overrightarrow{\mathrm{r}}-\overrightarrow{\mathrm{r}^{\prime}}\right|} \\
& \text { (d) } \mathrm{V}_{0} \mathrm{e}-\beta\left|\mathrm{r}-\mathrm{r}^{\prime}\right| \\
& \text { (e) } \mathrm{V}_{0}\left(\left|\overrightarrow{\mathrm{r}}-\overrightarrow{\mathrm{r}^{\prime}}\right| \leqslant \mathrm{R}\right)
\end{aligned}
$$

plus the standard spin, isotopic spin and tensor force term.

Up to 15 coupled equations can be handled at present; expansion to 25 is underway. Analog states can also be treated. (E. Auerbach, C. Dover, R. Lemmer)

\section{Excitation of Intrinsic and Rotational States in $\mathrm{Ne}^{20}$}

We have been looking at $\mathrm{T}=1$ excited states of $\mathrm{Ne}^{20}$ as analog states of resonances observed in $F^{19}(n, n) F^{19}$ total cross sections in the energy range 0 to $3 \mathrm{MeV}$ incident neutron energy. The ground state of $\mathrm{Ne}^{20}$ is.probably deformed. Therefore, we take as our model for $\mathrm{T}=1$ excitations, particle-hole excitations in a deformed potential well. To simplify the numerical aspects of the problem, single particle states in a deformed oscillator potential are employed. This permits us to use the "asymptotic" quantum numbers $\left\{n_{1} n_{11} m m_{s}\right\}$ where $n_{1}=$ quantum number for vibrations perpendicular to the nuclear symmetry axes, and $n_{11}$ has the same meaning for vibrations along the symmetry axis. $\mathrm{m}$ and $\mathrm{m}_{\mathrm{s}}$ are the orbital and spin angular momentum projections along the symmetry axis. Our intrinsic Hamiltonian is

$$
\mathrm{H}=\mathrm{H}_{\mathrm{H} \cdot \mathrm{O}}+\mathrm{C} \underline{\ell} \cdot \underline{\mathrm{S}}+\underline{\mathrm{D}}^{2}
$$

in a reference frame rotating with the nucleus, and includes a spin-orbit interaction. We have diagonalized $\mathrm{H}_{\text {outer }}$ in the basis provided by $\mid\left(n_{1} n_{11} \mathrm{~m} \mathrm{~m}_{\mathrm{s}}\right)_{1},\left(\mathrm{n}_{1} \mathrm{n}_{11} \mathrm{~m} \mathrm{~m}_{\mathrm{s}}\right)_{\mathrm{n}}>, \mathrm{p}=$ particle, $\mathrm{h}=$ hole, to get states of good total spin projection $\Omega_{p h}=\Omega_{p}-\Omega_{h}$ along the nuclear symmetry axis, using a standard two-body interaction $\mathrm{V}_{12}=\left(\mathrm{a}+\mathrm{b} \vec{\sigma}_{1} \cdot \frac{\mathrm{p}}{\sigma_{2}}\right) \delta\left(\overrightarrow{\mathrm{r}}_{1}-\overrightarrow{\mathrm{r}}_{2}\right)$.

We are presently exploring the effects of the rotational motion of the nucleus on the excitation of these particle-hole states by particle scattering from an $A=19$ target. The deformed 
field that the incident nucleon feels allows it to "rotate" the nucleus simultaneously while exciting a particle-hole pair; i.e., a rotational band can be produced on the intrinsic particle-hole excitation. Calculations of the particle escape widths for the $\mathrm{T}=1, \mathrm{~J}=0^{-}, 1^{-}, 2^{-}$states in $\mathrm{Ne}^{20}$ have been completed for decay to both the ground state and excited states of $\mathrm{F}^{19}$, which have a rotational character. Total widths vary between 1 to $300 \mathrm{keV}$ depending on the energy and character of the states involved. The total cross section and differential cross section is being calculated in order to compare with recent measurements of the total neutron in $F^{19}$. Excitation of the $T=1, S=1^{-}$ states in the $F^{19}(p, \gamma) \mathrm{Ne}^{20}$ reaction is also under study. (I. Afnan and $\mathrm{R}$. Lemmer)

\section{Resonance Scattering of Neutrons in the Extended Shell Model}

Calculations along lines described in previous progress reports have been completed. A set of model-closed channel states has been constructed by diagonalizing the model Hamiltonian described in the May 1964 report in a subspace spanned by states in which one 3-phonon and between one and three $2+$ phonons are coupled with a neutron in one of the orbitals $2 \mathrm{p}_{3} / 2,1 \mathrm{f}_{5} / 2$, $2 \mathrm{p}_{1} / 2,1 \mathrm{~g}_{9} / 2,2 \mathrm{~d}_{5} / 2,3 \mathrm{~s}_{1} / 2,2 \mathrm{~d}_{3} / 2$ to total angular momentum and parity $\frac{1}{2}+$. The diagonalization has been carried out and escape widths of the model states calculated in the compound nuclei $\mathrm{Ni}^{61}, \mathrm{Ge}^{71}$, and $\mathrm{Se}^{77}$. The uncertainties in the numerical values of the escape widths are fairly large; the sources of these uncertainties have been studied in some detail. A simple upper bound on level shifts has been devised.

In $\mathrm{Ni}^{61}$ the density of states with $\mathrm{J}=\frac{1}{2}+$ near the neutron emission threshold is too small to justify an attempt to describe the mixing of the model states into more complicated configurations. It is therefore not feasible to extract a neutron strength function for the small energy intervals over which data is presently available. Level densities in $\mathrm{Ge}^{71}$ and $\mathrm{Se}^{77}$ are higher and the possibility of reproducing experimental strength function values for these nuclei in terms of a damping width has been investigated. A truly meaningful comparison of the calculated values with experiment is thwarted by our inability to predict positions of model states with sufficient accuracy.

The problem of working with an incomplete set of doorway states, which arises in the context of the model used, has been treated in the case that a damping width is a valid parametriza tion of the mixing into more complex states.

This investigation has now been completed and is being written up as the thesis of H. Picker. (H. Picker, R. H. Lemmer) 
LIX. An Optical Potential with Intermediate Phonons

An intermediate phonon theory of the nuclear reactions has been developed to calculate vibrational transition amplitudes in the framework of an optical model. It satisfies the requirement of the Blair-phase rules. (W. Bassichis, H. Feshbach, and J. Reading)

\section{Optical Model}

The optical model at high energies has been studied in an attempt to explain why empirically the imaginary part of the optical model potential is $50 \%$ smaller than that calculated. The Glauber approximation has been compared to the Watson Potential, and their differences elucidated. Hard core correlations have been calculated. The impulse approximation has been reformulated and large errors found in it with the accepted nucleon-nucleon forces. How ever, all these effects make the agreement in theory and experiment.worse. Correlation effects with a correlation function are hard to calculate. However, the ratio of the effect on the real and imaginary parts of the potential can be estimated to a fair degree of accuracy and then the real part is theoretically affected much more than the imaginary part, a situation not in agreement with experiment. Important effects are due to the non-locality of optical potential. The high energy approximation has been extended to include this and excellent agreement has now been obtained with experiment. (J. F. Reading)

\section{Study of the Scattering Matrix for Inelastic Nucleon Scattering}

The scattering matrix for a nuclear interaction with many closely coupled channels is considered in a model which replaces all contributions due to closed channels by a complex poten tial. The coupling between channels is also given by square wells. The strenth of the coupling terms is assumed to be random. It is shown that if the Hamiltonian matrix elements are generated according to a distribution which maintains representational invariance, the distribution function for the reduced widths is completely determined, such that on the average the reduced width matrix elements vary inversely with the number of channels closely coupled. The widths at the poles of the scattering matrix in the intermediate energy region are computed, using realistic values for the inelastic energy level spacing and strength of the square well. They are found to be on the order of a few $\mathrm{keV}$ at a nucleon energy of around $25 \mathrm{MeV}$ for zero imaginary potential. The inelastic cross section is computed, and, for zero imaginary potential is found to be approximately one-third of the experimental value. Considering that a square well potential is expected to give too small an inelastic cross section due to the high reflectivity of such potentials, this agreement shows that a good portion of the inelastic scattering is due to contributions from the closelycoupled open channels. (V. Newton and H. Feshbach) 


\section{Pauli -P rinciple in Deuteron Stripping}

The effect of the identity of nucleons on stripping of deuterons (or pick-up) is being examined. Using simplified models it appears possible to determine the appropriate projection operator. We plan to treat the resultant equation by the DWBA method, at least.initially. (L. Dohnert and H. Feshbach)

LXIII. Intermediate Structure in $\mathrm{Ni}^{58}\left(\mathrm{p}, \mathrm{p}^{\prime}\right) \mathrm{Ni}^{58}$

Analysis of the reaction $\mathrm{Ni}^{58}\left(\mathrm{p}, \mathrm{p}^{\prime}\right) \mathrm{Ni}^{58}$ at $7-10 \mathrm{MeV}$ is in progress. The coupling of the $\mathrm{g}_{9} / 2$ single particle resonance to the collective states is being used to explain the observed resonance structure. (A. Stamp)

XLIV. Analytic Properties of Two and Four Point Function of a Many Body System

An exactly soluble many body model has been set up for the study of the analytic properties of two and four point functions. The results for the four point function show besides normal thresholds the appearance of anomalous thresholds below the branch point of the two body continuum, generated by an outgoing state of two bound particles. Resonances of the N+1 particle system have been shown to generate branch points in the complex energy plane. Further study on the $\mathrm{S}$-matrix of the system is in progress. (H. D. Dahmen)

\section{Scattering Problem with Non-Local Potentials}

A study is being made of the scattering problem using the Schroedinger equation for a non-local Hartree-Fock potential. The Brueckner-Gammel-Weitzner method, used for the bound state problem, does not appear to converge, and studies of the convergence properties of a straightforward iteration of the Schroedinger equation are in progress. (A. K. Kerman and A. D. MacKellar)

\section{Separable Potentials}

- The inverse problem for separable potentials has been studied. It is non-unique, and can only be resolved by looking at $n$-body interactions. The analytic properties derived for the potentials have been used to give an alternative proof of the Perey effect. (J. F. Reading) 


\section{Pauli Principle and Nuclear Reactions}

The effect of the Pauli principle and exchange on nucleon-nucleus scattering reactions has been studied. Techniques have been developed for truncating a set of coupled channel equations, and for properly treating channel amplitudes, in view of these effects. Of special concern have been questions of non-independent bases; these are the primary source of complication in anti-symmetrized representations. Channel Hamiltonians and inter-channel coupling potentials, corrected for Pauli effects, have seen proposed to parallel these expressions which were given earlier ${ }^{1}$ in the absence of exchange and antisymmetrization. From these new terms, an effective single particle equation has been derived, following the identical steps used in the earlier paper. A model calculation involving the new techniques has been undertaken for the scattering of neutrons from $\mathrm{O}^{16}$, drastically simplified, however, for easy illustration of the exchange correction features. (W. Friedman and H. Feshbach)

\section{Construction of P rojection Operators for Nuclear Reactions}

It is shown that projection operators on the open channels can be defined for a) re-arrangement collisions without anti-symmetrization and b) inelastic collisions with anti-symmetrization. The properties of these operators are discussed and the coupled channel equations derived. (M. Coz)

\section{Optical Model Studies}

A program has been written to calculate the cross section, pỏlarization, depolarization, rotation and asymmetry for neutrons scattered by nuclei. It includes the usual optical model potentials plus an $\vec{I} \cdot \vec{s}$ term. The spin-spin interaction splits the single particle resonances and the elastic scattering shows structure of the absorption is small. The deformation is predicted to be negative for angles near the diffraction minima. (A. Stamp)

\section{Center of Mass Effects in Nuclear Reaction Theory}

The Feshbach theory of nuclear reactions is being extended to include center of mass effects. The connection to the Hartree-Fock approxima tion must then be extended so that itbecomes related to the generalized Hartree-Fock theory of Kerman and Klein. (A. Jaffrin and A. K. Kerman)

1. Ann. Phys. 5, 357 (1958). 


\section{Theory of Nuclear Resonance Reactions}

The relationships between the poles and residues of the S-matrix on the one hand, and the eigenvalues and eigenfunctions of the associated discrete spectrum ( $R$-matrix) on the other hand, were investigated analytically. A new many-level formalism was developed for this purpose which, instead of the usual finite number of levels, permits one to take an unlimited number of resonance levels into account. Among the questions investigated were the effects of changing channel radii and boundary conditions, of using finite numbers of resonance levels, as well as the relation between resonance and direct processes. The effects of intermediate structure con figurations on cross sections were investigated, with particular regard to the effects of the resulting correlations between channel amplitudes. (P. A. Moldauer)

\section{Inelastic Scattering}

The study of a microscopic description of inelastic scattering has been continued (in collaboration with N. Glendenning from the University of California). The microscopic form factors have been calculated for the nickel and tin isotopes. The effects of configuration mixing on these form factors are very important. The microscopic form factors are broader than the macroscopic ones; they can have quite different shapes and they peak inside the nuclear surface. The influence of the radial form of the individual wave functions, and of the optical parameters on the angular distribution is under investigation. (M. Veneroni)

\section{Channel Theory of the Fission P rocess}

The number of exit channels can be determined from the analysis of neutron resonances in two ways, either by the fluctuations of the fission widths, $\Gamma_{f}$, or by the average value $\left\langle\Gamma_{f}>/ D\right\rangle$ assuming the Wheeler formula is correct. But these two methods give very different results. For example, in the case of $U^{235}$, one obtains $\nu>4$ with the first method, and $\nu=0.25$ with the second one; that is to say, a ratio of 16 between the two values of $\nu$. It has been suggested that the omission of resonance interference effects could be the source of such a big discrepancy. We studied this question and arrived at the conclusion that this explanation can probably apply to $\mathrm{U}^{233}$ but not to $\mathrm{U}^{235}$ or $\mathrm{Su}^{239}$.

An invited paper on this subject was presented at the American Physical Society meeting at Washington. (A. Michaudon) 
LXXIV. P roperties of the Average Cross Section in the Case of.

Pure Elastic Scattering of Low Energy Neutrons by Nuclei

The actual cross section shows well separated resonances. When this cross section is suitably averaged over an energy interval much greater than the mean level spacing of the resonances, then we obtain a smooth variation as a function of the neutron energy which contains two terms: the potential scattering and a second term which is proportional to the strength function. But there is no correction proportional to the square of the strength function, though such a function correction has been found by several authors. In fact, this correction is in violation of the unitarity of the S-matrix. In addition to this, the unitarity of the S-matrix leads to some statistical properties of the resonance parameters. (A. Michaudon)

LXXV. Qualitative Aspects of the Neutron-Nuclear Interaction and the Optical Model

The occurrence of broad maxima and minima in the energy dependence of neutron-nuclear total and scattering cross sections below $100 \mathrm{MeV}$ is given a simple explanation. Within the framework of the Glauber diffraction approximation, the general conditions for the appearance of these maxima and minima are obtained in terms of an integral, over impact parameters, involving a phase shift function. A modification of the approximation is employed so that the formalism may be applied below $100 \mathrm{MeV}$. The assumption that the neutron wave suffers an average phase change due to the presence of the nucleus equal to an integral multiple of $\pi$ at cross section maxima or minima is shown to be not valid. Near regions of maxima, appreciable contributions come from. a number of partial cross sections, and the appearance of these maxima imply neither a resonance in any single partial wave nor a maximum in any single partial cross section. (V. Franco)

\section{Nuclear Reaction Theory for the Scattering of Composite Particles}

It is well known that the elastic.nucleon-nucleus scattering amplitude $T_{K}{ }^{\prime \prime} K$ may be written as a Bethe-Goldstone linked cluster series:

$$
\left.\mathrm{T}_{\mathrm{k}^{\prime} \mathrm{k}}=\mathrm{t}_{\mathrm{k}^{\prime} \mathrm{k}}+\sum_{\mathrm{n}}<0\left|\mathrm{a}_{\mathrm{k}^{\prime}(-)} \mathrm{V}\left(\frac{1}{\mathrm{E}_{0}+\epsilon_{\mathrm{k}}-\mathrm{H}_{0}} \mathrm{v}\right)^{\mathrm{n}} \mathrm{a}_{\mathrm{k}(+)}^{+}\right| 0\right\rangle
$$

where $\mathrm{H}_{0}$ is a Hartree-Fock (model) Hamiltonian, $\mid 0>$ an eigenstate of $\mathrm{H}_{0}^{\prime},-\overline{\mathrm{V}}=\mathrm{H}-\mathrm{H}_{0}, \mathrm{t}_{\mathrm{K}} \mathrm{K}_{\mathrm{O} t \text { ) }}$ the scattering amplitude in $\mathrm{H}_{0}$, and $\mathrm{a}_{\mathrm{k}}^{+}( \pm) \mathrm{a}_{\mathrm{K}}( \pm)$ are creation and annihilation operators of out) in) going continuum states of $\mathrm{H}_{0}$, of energy $\epsilon_{\mathrm{K}}$.

We have extended the method to describe scattering of composite particles, mainly deuterons.

The use of the model Hamiltonian $\mathrm{H}_{0}$ requires that the deuteron be expanded in eigen functions of $\mathrm{H}_{0}$; the deuteron creation operator $\mathrm{A}_{\mathrm{K}}^{+}$is 
Theoretical Group

$$
\mathrm{A}_{\mathrm{K}}^{+}=\sum_{\alpha \beta} \mathrm{C}_{\mathrm{K}}(\alpha \beta) \mathrm{a}_{\alpha}^{+} \mathrm{a}_{\beta}^{+}
$$

with $\mathrm{C}_{\mathrm{K}}(\alpha \beta)$ satisfying the Schroedinger equation

$$
\left(\epsilon_{\alpha}+\epsilon_{\beta}-\mathrm{E}_{\mathrm{K}}\right) \mathrm{C}_{\mathrm{K}}(\alpha \beta)+\sum(\alpha \beta|\mathrm{V}| \gamma \delta) \mathrm{C}_{\mathrm{K}}(\gamma \delta)=0
$$

We found that a generalized linked cluster series of the type of eq. (1) can be written for this case, and by suitable partial summation of this series, an optical potential for the deuteron may be defined. This work is near completion.

Difficulties arise in attempting to generalize this method to inelastic processes like pick up or stripping, since different eigenstates of $\mathrm{H}$ occur as initial and final states, and the elimination of unlinked clusters is not so trivial anymore. (B. Junkin and F. Villars)

\section{Deuteron-Deuteron Collisions at High Energies}

The Glauber-Moliere high-energy approximation has been used to investigate deuterondeuteron collisions. Total absorption and elastic scattering cross section and various angular distributions have been calculated, and numerical results for the limiting case of infinite incident deuteron energy are obtained. Double, triple, and quadruple scattering corrections are included. (V. Franco)

\section{Deuteron Stripping Theory}

High energy deuteron stripping has been studied using the Glauber approximation, in an attempt to explain results now available experimentally. The peaking of stripped.protons at forward angles is still unexplained, however the total cross sections and the angular distribution at larger angles is well understood. (J. F. Reading and V. Franco)

\section{Singular Potentials Obtained from the Gelfand Levitan Equation}

Non-locality is not necessary to obtain singular potentials. When it is needed this implies that the Hamiltonian cannot be reconstructed in a unique manner from the knowledge of its spectral function. (M. Coz) 


\section{CONFERENCES AND MEETINGS}

Addresses and Invited Papers

E. H. Auerbach, "Determination of Optical Potentials from Polarization Data", P roc. of International Conference on the Study of Nuclear Structure with Neutrons, Antwerp, Belgium, July $19-23,1965$

E. H. Auerbach, S. O. Moore, "Calculation of Inelastic Scattering of Neutrons in the MeV Region", P roc. of International Conference on Nuclear Structure with Neutrons, Antwerp, B elgium, July 19-23, 1965.

R. H. Lemmer, "Intermediate Resonance Structure in Nuclear Reactions", presented at the American Physical Society meeting, Jan. 27-30, 1965.

\section{Abstracts .}

C. Bressel, A. Kerman, E. Lomon, "Modified Hamada-Johnston Potential with Finite Cores", Am. Phys. Soc. meeting, New York (June 23-25, 1965).

H. P. Jolly, "T=2 Excitations in $\mathrm{O}^{16 ", ~ A m . ~ P h y s . ~ S o c . ~ m e e t i n g ~(J a n . ~ 27-30, ~ 1965) . ~}$

\section{PUB LICATIONS}

E. H. Auerbach, L. Rosen, J. G. B eery, and A. S. Goldhaber, "Nucleon-Nucleus Interactions", Nature 206, 2846 (1965).

E. H. Auerbach, L. Rosen, J. G. Beery, and A. S. Goldhaber, "Elastic Scattering of 10.5- and 14.5-MeV Polarized P rotons from Nuclei and the Optical Model Potential at Intermediate Energies", Ann.P hys. 34, 96-152 (1965).

W. P. Beres, "Vibrational States of Odd Mass Nuclei by the Quasi-Particle Method", Nucl. Phys. $68,49-85$ (June 1965).

J. B. Bronzan, "A Soluble Model Field Theory with Vertex Function", Phys. Rev. 139, 3B, (August 1965).

M. Coz, "P rojection Operators for Rearrangement Collisions", Ann. Phys. 35, No. 1, (Oct. 1965).

P. Federbush, "An Example of the Mandelstam Representation in P erturbation Theory", Ann. P hys. 33, 272 (1965).

P. Federbush, "Calculation of some Homology Groups Relevant to Sixth-Order Feynman Diagrams", J. Math. Phys. 6, 941 (1965).

P. Federbush, "Note on Non-Landau Singularities", J. Math. P hys. 6,825 (1965).

H. Feshbach and A. K. Kerman, "Studies of Hypernuclei with K Meson B eams", Preludes in Theoretical Physics, in honor of V. Weisskopf (1965).

D. Flamm, "The Electromagnetic Structure of Vector Mesons in $\mathrm{SU}_{3}$ Symmetry", Nuovo Cim. 38, 291 (1965).

V. Franco, "Qualitative Aspects of Neutron-Nuclear Interactions and the Optical Model", Phys. Rev. 140, No. 6B, (Dec. 20, 1965)

V. Franco, R. J. Glauber, "High Energy Deuteron Cross Sections", Phys. Rev. 142, 1195-1214 (Feb. 1966).

H. Goldberg and E. L. Lomon, "The Analytic Continuation of an $\mathrm{S}_{11} \mathrm{P}$ ion Nucleon Amplitude to the $P_{11}$ Amplitude", Nuovo Cim. 37, No. 3 (June 1965).

$\mathrm{K}$. Huang, "The $\mathrm{SU}_{3}$ Mass Formula", P reludes in Theoretical Physics, in honor of V. Weisskopf (1965).

K. Huang, F. E. Low, "Exact Bootstrap Solutions in Some Static Models of Meson-Baryon Scattering", J. Math. Phys. 6, No. 5 (May 1965).

K. Huang, A. H. Mueller, "Exact Bootstrap Solutions to the Low Equation", Phys. Rev. 140, No. 2B, (Oct. 25, 1965).

K. Huang and A. H. Mueller, "Non-Existence of 'P ure Bootstrap' Solutions to the Chew-Low Theory", Phys. Rev. Lettèrs 14, No. 11 (March 1965). 
K. Huang, A. C. Olinto, "Quantized Vortex Rings in Superfluid Helium, A P henomenological Theory", Phys. Rev. 139, No. 5A (August 1965).

H. P. Jolly, "Inelastic Electron Scattering Cross Sections and Odd-Parity Excitations of Ca ${ }^{40 "}$, Nucl. P hys. 67 (May 1965).

A. K. Kerman, D. M. B rink and A. F. R. de Toledo Piza, "Interval Rules and Intensity Ratios in Vibrating Spherical Nuclei", Phys. Letters 19, No. 5, 413-415 (15 Nov. 1965).

A. K. Kerman, L. Cilenza, and A. Klein, "Collective Motion in Finite Many-Particle Systems. III. Foundations of a Theory of Rotational. Spectra of Deformed Nuclei", Phys. Rev. 140, 2B, B 245-B263 (Oct. 1965).

A. K. Kerman and A. Klein, "Collective Motion in Finite Many-Particle Systems II", Phys. Rev. 138, 5B, B $1323-\mathrm{B} 1332$ (June 1965).

T. K. Kuo and T. Yao, "Electromagnetic Mass Splittings in the SU(6) Symmetry Scheme", Phys. Rev. Letters 14 , No.3, 79 (1965).

R. K. Logan, "A Single Regge Pole Analysis of $\pi$-p Charge Exchange Scattering", Phys. Rev. Letters 14, No. 11, (March 1965).

R. K. Logan, "Possible Discrepancy between the Regge-Pole Hypothesis and the Difference of the $\pi^{+} p$ and $\pi^{-}$p Differential Cross Sections", Phys. Rev. Letters $\underline{14}$, No. 22 (May 1965).

F. Low, "Are Wave Functions Finite", Preludes in Theoretical Physics, in honor of V. F. Weisskopf. (1965)

F. E. Low, "Heavy Electrons and Muons", Phys. Rev. Letters 14, No. 7 (Feb. 1965).

H. Mitter, "Nonlinear Quantum Field Theories at Small Distances", Nuovo Cim. 38, (July 16, 1965).

R. P errin, "Infra-Red B ehavior of the Wave Function Renormalization Constant in Covariant Gauges", Nuovo Cim. 38 (Aug. 16, 1965).

R. Perrin, "Relation between Poles in P ropagators and the Existence of Particles", Phys. Rev. 140, 1B (Oct. 11, 1965).

R. Perrin, E. L. Lomon, "Infrared Contributions to Intermediate State Sums in Quantum Electrodynamics", Ann. P hys. 33 (July 1965).

R. F. Sawyer, "Johnson-Treiman Relations from Vector Meson Exchange", Phys. Rev. Letters 14 , No. 12 (March 1965).

R. F. Sawyer, "Three Body Forces in the N $\pi \pi$ System", Phys. Rev. 139, $1 B$ (July 12, 1965).

J. Reading, "Deuteron-Carbon Elastic Scattering at $650 \mathrm{MeV}$ " (with L.M.C. Dutton, J. D. Jafar, H. B. Van Der Raay, D. G. Ryan, J. A. Stiegelmair, R. K. Tandon), Phys. Letters 16, 331 (1965).

C. Shakin and Y. R. Waghmare, "Foundations for Hartree-Fock Calculations with Singular Potentials", Phys. Rev. Letters 16, 403 (1966).

F. Villars, "Elementary Quantum Theory of Nuclear Collective Rotation", Nucl. Phys. 74, 353 (1965).

\section{THESES}

C. N. Bressel, "Nucleon-Nucleon P otentials with Finite Repulsive Cores", Ph.D., (May 1965).

W. J. de Bonte, "K-N Interaction for the I-O State in the Boundary Condition Model", B.S. (June 1965).

R. C. Goldstein, "Determination of Single Particle Escape Widths in Photonuclear Reactions", B. S. (June 1965).

W. J. Holman, III, "Modified Isobar Approximation for Pion-Rho Scattering", Ph.D. (Feb. 1965).

A. H. Huffman, "A Boundary Condition Model for the $P_{33}$ Pion-Nucleon State", M.S. (June 1965).

R. K. Logan, "A P henomenological Study of High Energy $\pi p$ Elastic and Charge Exchange Scattering", Ph.D. (August 1965).

A. I. Miller, "Three Channel Boundary Condition Model for the Second Pion-Nucleon Resonance", Ph.D. (August 1965).

P. K. Mitter, "Three Particle States in the Octet Model of Unitary Symmetry", Ph. D. (June 1965).

A. H. Mueller, "Static Model of Meson Baryon Scattering", Ph.D. (June 1965).

A. C. Olinto, "Vorticity in a Superfluid", Ph.D. (July 1965). 
H. K. Quang, "On the Three-Nucleon Potential", Ph.D. (August 1965).

E. Salz, "N as a Bound State of $\mathrm{N}^{*}$ and $\pi^{\prime \prime}, \mathrm{Ph}$. D. (Oct. 1965).

J. P. Svenne, "A Hartree-Fock Calculation for Finite Nuclei with a Non-Local Two-Body Potential", Ph.D. (August 1965).

P. C. M. Yock, "High Energy Scattering at Low Momentum Transfer", Ph.D. (August 1965). 


\section{P ERSONNEL LISTING B Y P ROJECTS}

(as of December 31, 1965)

\section{Visiting Committee}

P rof. H. E. Gove, co-chairman Department of Physics University of Rochester Rochester, New York

Prof. K. Greisen, co-chairman Laboratory of Nuclear Studies Cornell University Ithaca, New York

Prof. W. A. Fowler Physics Department California Institute of Technology Pasadena 4, California

Dr. R. F. Taschek Los Alamos Scientific Laboratory Los Alamos, New Mexico

P rof. D. H. Wilkinson Nuclear P hysics Laboratory Oxford University Oxford, England

Dr. C. N. Yang Physics Department State University of New York Stony B rook, L.I., New York
MIT Interdepartmental Laboratory Committee for the Laboratory for Nuclear Science

P rof. P. T. Demos

Professor of Physics

Director of the Laboratory

P rof. M. B enedict $P$ rofessor of Nuclear Engineering Head of the Department

Prof. W. W. Buechner $P$ rofessor of $P$ hysics Head of the Department

P rof. John Ross $P$ rofessor of Chemistry Head of the Department

Prof. J. B. Wiesner Institute $P$ rofessor Dean of the School of Science

\section{Laboratory Committees}

\section{Computer Committee}

P rof. I. A. Pless, chairman

Prof. B. T. Feld

Prof. H. Feshbach

$P$ rof. R. I. Hulsizer, Jx.

Prof. L. S. Osborne

\section{Reading Room Committee}

Prof. B. T. Feld, chairman

P rof. A. B ernstein

P rof. M. Deutsch

Prof. J. I. Friedman

P rof. G. Garmire

$P$ rof. K. Johnson

P rof. A. K. Kerman
Inner Space Committee

Prof. D. H. Frisch, chairman

Prof. W. W. Buechner

P rof. M. Deutsch

$P$ rof. B. T. Feld

P rof. H. Feshbach

\section{Safety Committee}

$P$ rof. L. Rosenson, chairman

J. Kuchta, safety officer

B. Bailey

B. Wadsworth

P. T. Demos

M. Dondero

Dr. Harriet Hardy

R. L. Loria 
P rof. P. T. Demos

Director

Dr. F. J. Eppling

Associate Director

R. L. Loria

Headquarters

B. Bailey

Engineering

R. W. Calileo

General Services
E. DeAgazio

Electronics Shop

R. Gustavson

Machine Shop

J. L. Sargeant

Purchasing

A. J. Scully

Accounting

S. A. Tardivo

Property

C. W. Tourtellotte

Drafting

RESEARCH GROUP S

Cosmic Ray Group

Research Staff

Prof. B. B. Rossi

Prof. H. S. Bridge

Prof. H. Bradt

Dr. J. Davis

*Dr. A. Egidi University of Rome

Dr. V. Formisano University of Rome

P rof. G. Garmire

*P rof. W. L. Kraushaar University of Wisconsin

P rof. A. J. Lazarus

*Dr. M. La P ointe University of Maryland

Dr. W. Lewin

Dr. J. D. Linsley

Dr. G. Moreno

*Dr. M. Oda Institute of Nuclear Study University of Tokyo

P rof. S. Olbert

P rof. J. Overbeck

Dr. L. Pai

*Dr. V. A. Sarabhai Physical Research Lab. Navrangpura, Ahmedabad, India
*P rof. F. Scherb

University of Wisconsin

W. B. Smith

Dr. G. Spada

Dr. B. V. Sreekantan

*Dr. M. Wada

Institute of Physical and Chemical Research

- Tokyo, Japan

Dr. E. F. Lyon, III

(on leave)

Graduate Students

J. Binsack

K. Brecher

D. Downes

F. Floyd

I. Glass

P. Higbie

$\mathrm{H}$. Hinteregger

A. Klimas

K. Kolling

A. Krieger

W. Mayer

S. Rappaport
J. Stein

R. Sullivan

R. Talbot

V. Vasyliunas

A. Womack

Undergraduates

E. Anderson

R. Chang

K. Chu

M. Coffey

T. Daily

H. Davitian

J. Elliott

C. Forline

D. Grimes

T. Gull

H. Ha rounian

S. Liv

S. Morganstein

P. Oddone

D. P epperberg

G. Ricker

P. Scheffler

D. Slevin

P. Smrz

V. Sukhatme

R. Thompson

R. Williams 
Research Staff

Dr. P. Bastien

C. B rooks

S. B rudno

Prof. B. T. Feld

Dr. V. Fischer

Prof. R. Hulsizer

Dr. D. Miller

Prof. I. Pless

Dr. A. Rogers

Prof. L. Rosenson

B. Wadsworth

Dr. T. Watts

P rof. R. Yamamoto

\section{$\underline{\text { Linear Accelerator Group }}$}

Research Staff

Prof. P. T. Demos

P rof. W. Bertozzi

P rof. S. Kowalski

Dr. C. P. Sargent

Dr. W. Turchinetz

\section{Visitors}

Dr. C. Bordner

H. Crouch

*P rof. Y. Eisenberg

J. Harris

Dr. H. Nagel

*Dr. E. Ronat

*Dr. A. H. Rosenfeld

Dr. L. Ventura
Graduate Students

D. Barton

B. Brabson

D. Brick

L. Kirkpatrick

B. Nelson

M. Partovi

J. Wolfson

\section{Undergraduate Students}

I. Asher

H. Bozler

W. Graves
Graduate Students

F. Hanser

J. Matthews

${ }^{*}$ G. Mutchler

T. P hillips

*E. Reinhardt

J. Bergstrom

M. Zombek

G. Hurford

High Energy Accelerator Physics Group

$\underline{\text { Research Staff }}$

Dr. R. Alvarez

Prof. J. I. Friedman

Prof. D. H. Frisch

Dr. D. Garelick

Dr. G. Glass

Dr. S. Homma

$P$ rof. H. Kendall

Dr. D. Luckey

Dr. P. Mockett

Prof. L. S. Osborne

Dr. J. Uglum

Dr. M. Wahlig

\section{Visitors}

Dr. Z. Bar-Yam

Dr. W. Kern

\section{Graduate Students}

D. B ellenger

M. B reidenbach

A. B roadfoot

A. Buffington

G. Cooperstein

J. Elias

V. Elings

O. Fackler

S. Gray

G. Hartman

P. Kirk

R. Lewis

E. Shibata

S. Smith

L. Sogard

L. Sompayrac

L. von Speybroeck

C. Ward

\section{*Terminated}


Research Staff

Prof. T. A. Belote

Prof. W. W. Buechner

H. Y. Chen

Prof. W. E. Dorenbusch

P rof. H. A. Enge

Dr. F. J. Eppling

*Dr. Ole Hansen

Rutgers, Copenhagen

$P$ rof. J. Kuperus

Prof. W. H. Moore

*Dr. D. J. Pullen

University of Penna.

P rof. J. Rapaport

A. Sperduto

\section{Visitor}

*A. Graue University of Bergen B ergen, Norway

\section{Graduate Students}

E. R. Chalbaud

E. R. Cosman

H. A. Ismail

G. K. Nair

M. N. Rao

${ }^{*}$ G. K. Schlegel

D. L. Smith

J. E. Spencer

Mrs. H. J. Young
Undergraduate Students

F. T. Dao

*M. A. M. Keehner

R. L. Kusper

J. L. Mikesell

*J. A. Otteson

D. N. Schramm

*S. T. Scott

${ }^{*}$ M. A. Skerry

*A. H. Sparer

H. A. White

*Louise Wilson

B. A. Wolff

\section{Radioactivity Group}

\section{Research Staff}

P rof. R. D. Evans

Prof. L. Grodzins (on leave)

Dr. S. G. Cohen (on leave)

Dr. H. W. Kraner

Dr. W. R. Neal

\section{Graduate Students}

J. Alonso

A. Buyrn

Y. W. Chow

A. R. Lewis

D. Murnick

G. Pramila

W. Schick

D. Yeboah-Amankwah

\section{Undergraduate Student}

M. Schuster

\section{Visitors}

T. R. Ophel

J. Alster

Northeastern Univ.

E. F. White

Dr. W. J. Kossler

Dr. K. Nagatani
Undergraduate Students

${ }^{*} \mathrm{G}$. Sample

M. Shork

\section{Graduate Students.}

R. Ginaven

E. Martens

E. P. Lippincott

S. M. Smith

${ }^{*} \mathrm{H}$. Tannenbaum

M. Slade

T. Ho

*Terminated 


\section{Research Staff}

Prof. V. F. Weisskopf

P rof. H. Feshbach

Dr. E. Auerbach

Dr. W. Bassichis

P rof. J. Bronzan

Dr. M. Cassandro

${ }^{*}$ Dr. M. Coz

Dr. H. D. Dahmen

*P rof. A. de-Shalit

P rof. P. Federbush

Dr. D. Flamm

*Dr. V. Franco

Dr. J. Greenman

P rof. K. Huang (on leave)

*Dr. J. Irwin

P rof. K. Johnson

*Dr. P. Jolly

Dr. G. Jona -La sinio

Prof. A. Kerman

Prof. R. Lemmer

Dr. B. Levy

Prof. E. Lomon (on leave)

P rof. F. Low

Dr. A. MacKellar

*Dr. A. Michaudon

*Dr. P. A. Moldauer

Dr. D. Pal

Dr. M. Pal

*P rof. C. A. Pearson

${ }^{*}$ P rof . D. C. P easlee

Dr. J. Reading

*Dr. G. Roepstorff

Prof. M. Ross

*P rof. R. Sawyer

P rof. C. Shakin

*Dr. D. Sprung

Dr. J. Svenne

Dr. A. Stamp

P rof. V. Teplitz

P rof. F. Villars

*Dr. M. Veneroni

Dr. Y. Waghmare

Dr. T. Yao

Dr. P. Yock

Dr. C. Zemach
Visitor

Dr. R. Evrard

Graduate Students

A. R. Afnan

M. L. Blackmon

G. D. Blum

R. A. B randt

R. W. B rown

G. E. Cooper

J. D. Cornwell

A. F. R. de Toledo Piza

C. B. Dover

W. A. Friedman

M. J. Feigenbaum

D. F. Freeman

J. C. Goldstein

S. Klawansky

K. A. Kolling

J-P. A. Lebrun

F. M: Newman

L. R. P eterson

E. F. Redish

N. C. Rogerson

R. M. Saenger

E. Salamin

L. M. Saunders

E. J. Schreier

M. P. Tomaselli

N. M. Toml janovich

P. B. Ulrich

G. M. Weingerger

R. K. P. Zia

*Terminated 
